\title{
Fluorocyclopropane-Containing Proline Analogue: Synthesis and Conformation of an Item in the Peptide Chemist's Toolbox
}

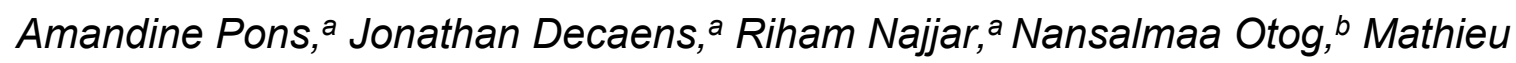
Arribat, ${ }^{,}$Sandrine Jolly, ${ }^{c}$ Samuel Couve-Bonnaire, ${ }^{a}$ Muriel Sebban, ${ }^{,}$Gael Coadou, ${ }^{a}$ Hassan Oulyadi ${ }^{\star},{ }^{a}$ David Speybrouck, ${ }^{c}$ Seiji Iwasa, ${ }^{b}$ André B. Charette, ${ }^{d}$ Thomas Poisson $^{a, e}$ and Philippe Jubaultta

a Normandie Univ, INSA Rouen, UNIROUEN, CNRS, COBRA (UMR 6014), 76000 Rouen, France.

b Department of Applied Chemistry and Life Science,Toyohashi University of Technology 1-1 Hibarigaoka, Tempaku, Toyohashi, Aichi 441-8580, Japan.

${ }^{c}$ Analytical Sciences - Discovery Sciences, Janssen Research \& Development, a Division of Janssen-Cilag, Campus de Maigremont, CS10615, F-27106, Val de Reuil Cedex, France

${ }^{d}$ Université de Montréal, Centre in Green Chemistry and Catalysis, Department of Chemistry, 1374, av. Thérèse Lavoie-Roux, Montréal, QC H2V 0B3, Canada.

${ }^{e}$ Institut Universitaire de France, 1 rue Descartes, 75231 Paris, France. 
Contents

NMR spectra and HPLC analysis

Structural determination of ((-)-11)

Crystallographic data of ((-)-10)
Page

S4-S47

S48-S66

S67-S83 
Olefins $\mathbf{1 a}$ and $\mathbf{1} \mathbf{b}$ were prepared according to described procedures. ${ }^{1}$

${ }^{1}$ For 1a see : Pons, A. ; Ivashkin, P. ; Poisson, T. ; Charette, A. B. ; Pannecoucke, X. ; Jubault, P. Chem. Eur. J. 2016, 22, 6239-6242. For 1b see : Guérin, D.; Gaumont, A.-C.; Dez, I.; Mauduit, M.; Couve-Bonnaire, S.; Pannecoucke, X. ACS Catal. 2014, 4, 2374-2378. 
NMR spectra and HPLC analysis

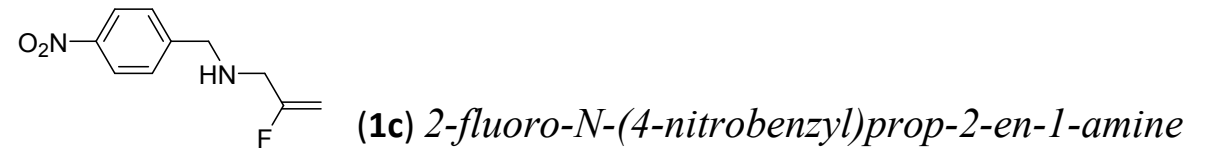

Figure S1. ${ }^{1} \mathrm{H}$ NMR spectum of 1 c $\left(300 \mathrm{MHz}, \mathrm{CDCl}_{3}\right)$.

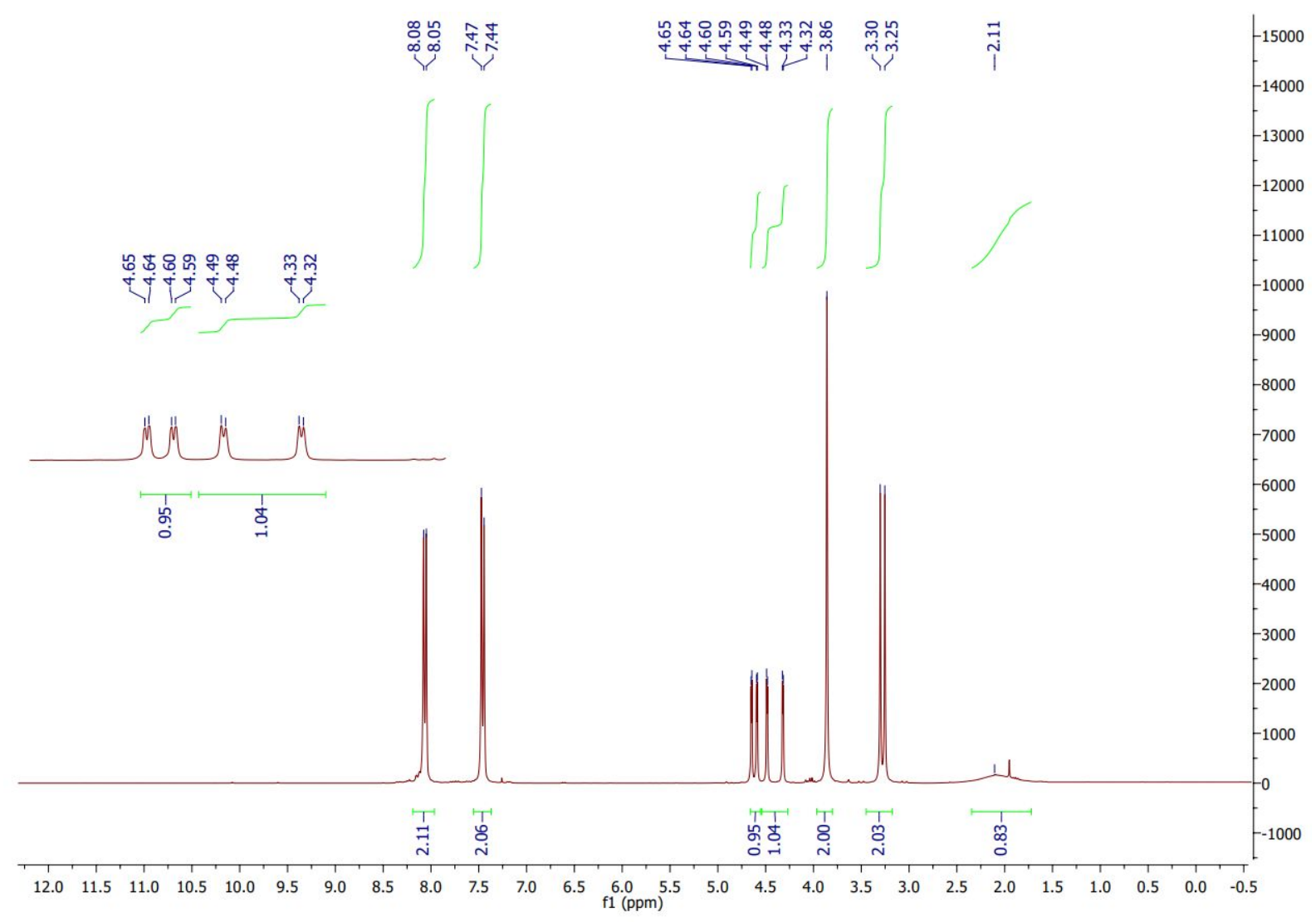


Figure S2. ${ }^{13} \mathrm{C} \mathrm{NMR}\{1 \mathrm{H}\}$ spectrum of $1 \mathrm{c}\left(75 \mathrm{MHz}, \mathrm{CDCl}_{3}\right)$.

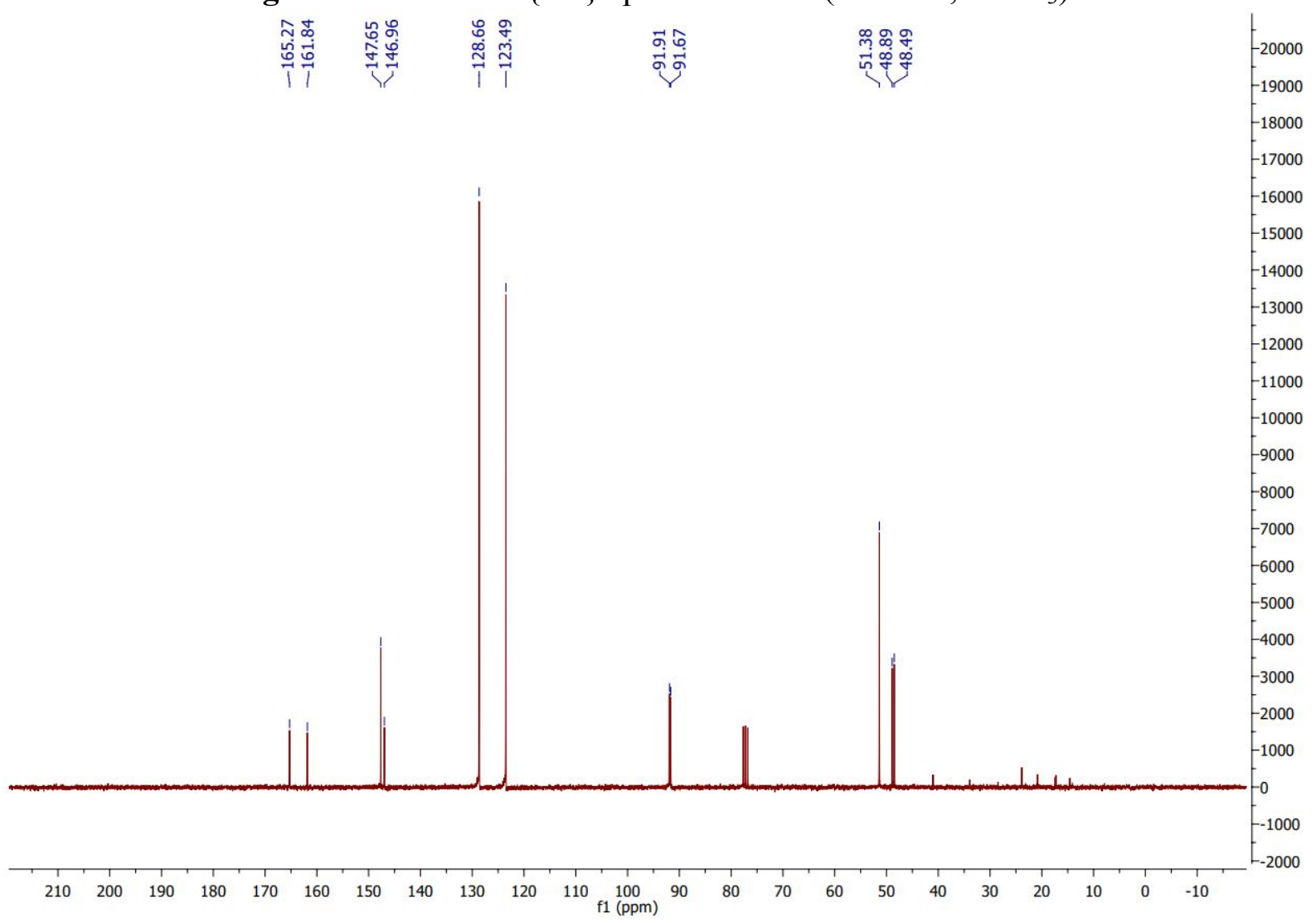

Figure S3. ${ }^{19} \mathrm{~F}$ NMR spectrum of $1 \mathbf{c}\left(282 \mathrm{MHz}, \mathrm{CDCl}_{3}\right)$.

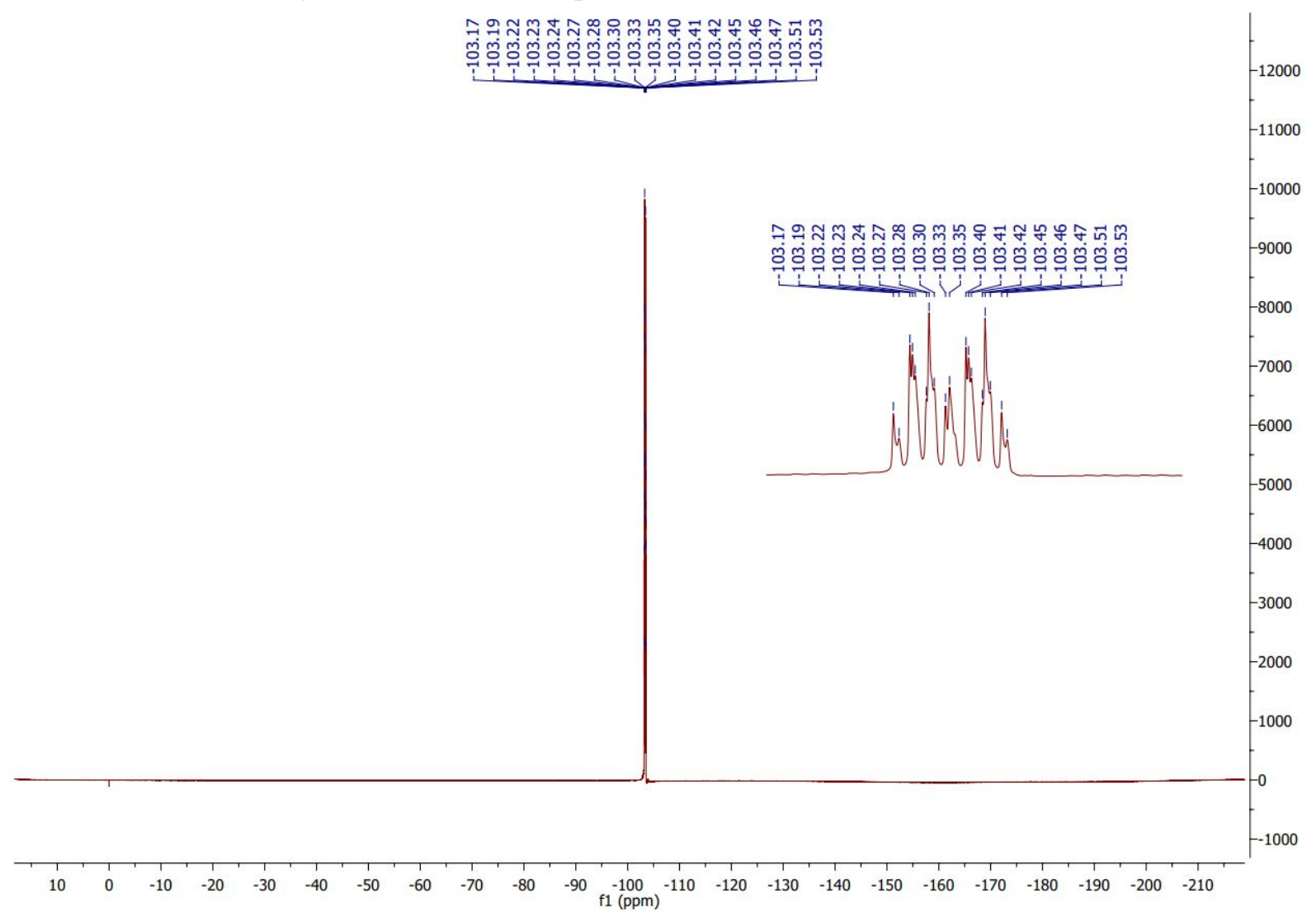




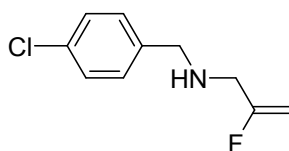

(1d) $\mathrm{N}$-(4-chlorobenzyl)-2-fluoroprop-2-en-1-amine

Figure S4. ${ }^{1} \mathrm{H}$ NMR spectrum of $1 \mathbf{d}\left(300 \mathrm{MHz}, \mathrm{CDCl}_{3}\right)$.

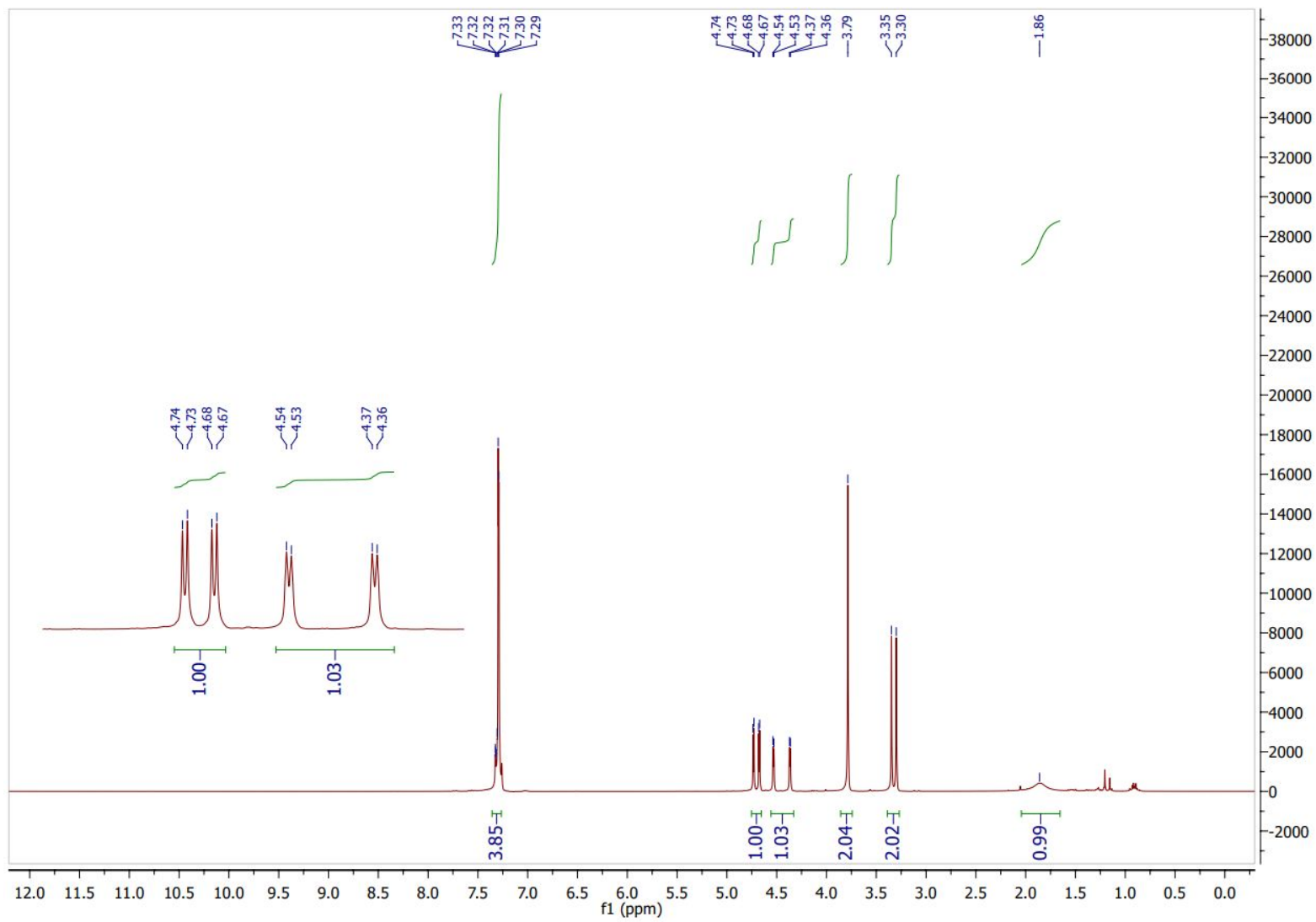


Figure S5. ${ }^{13} \mathrm{C} \mathrm{NMR}\{1 \mathrm{H}\}$ spectrum of $1 \mathbf{d}\left(75 \mathrm{MHz}, \mathrm{CDCl}_{3}\right)$.

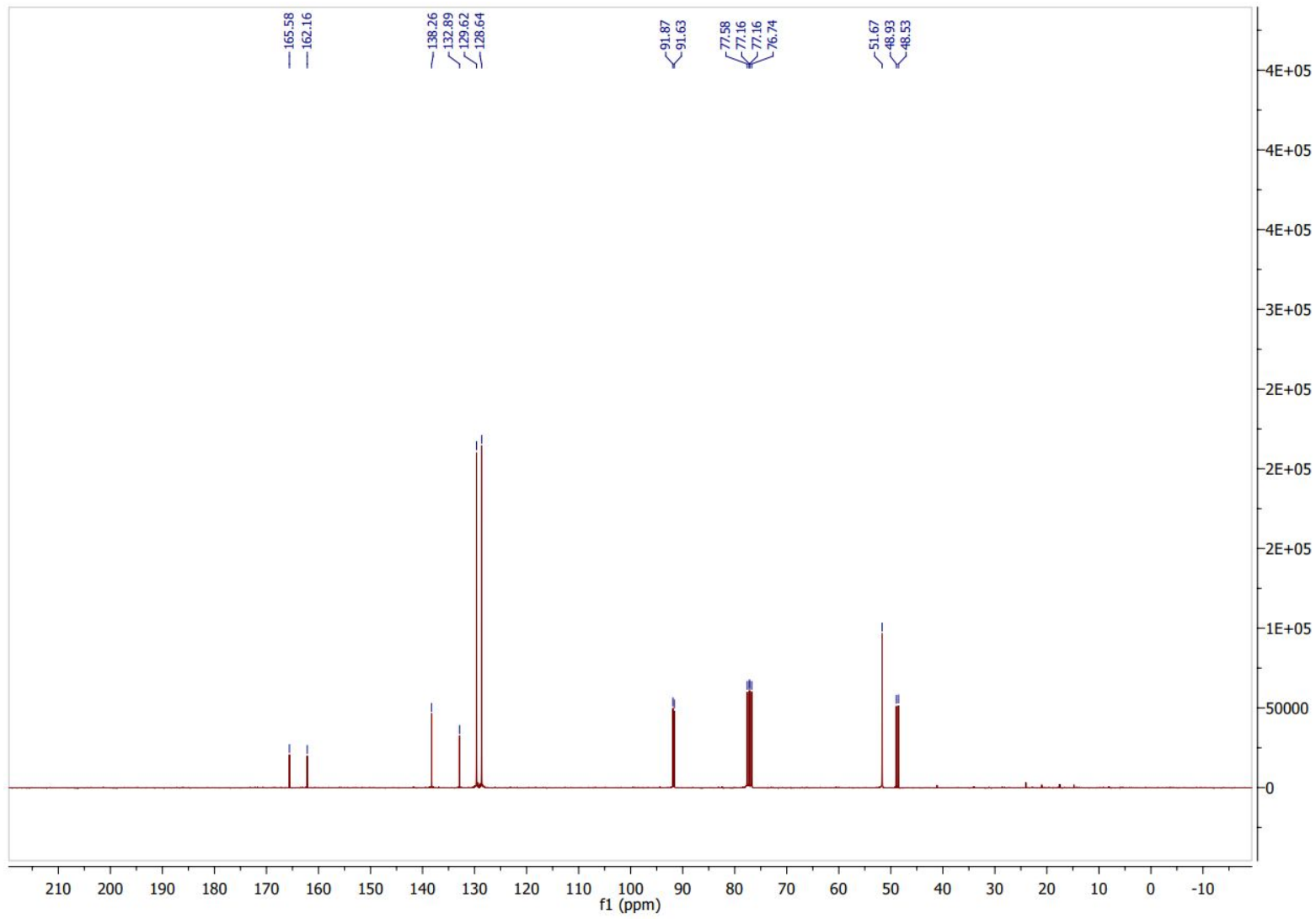

Figure S6. ${ }^{19} \mathrm{~F}$ NMR spectrum of $\mathbf{1 d}\left(282 \mathrm{MHz}, \mathrm{CDCl}_{3}\right)$.

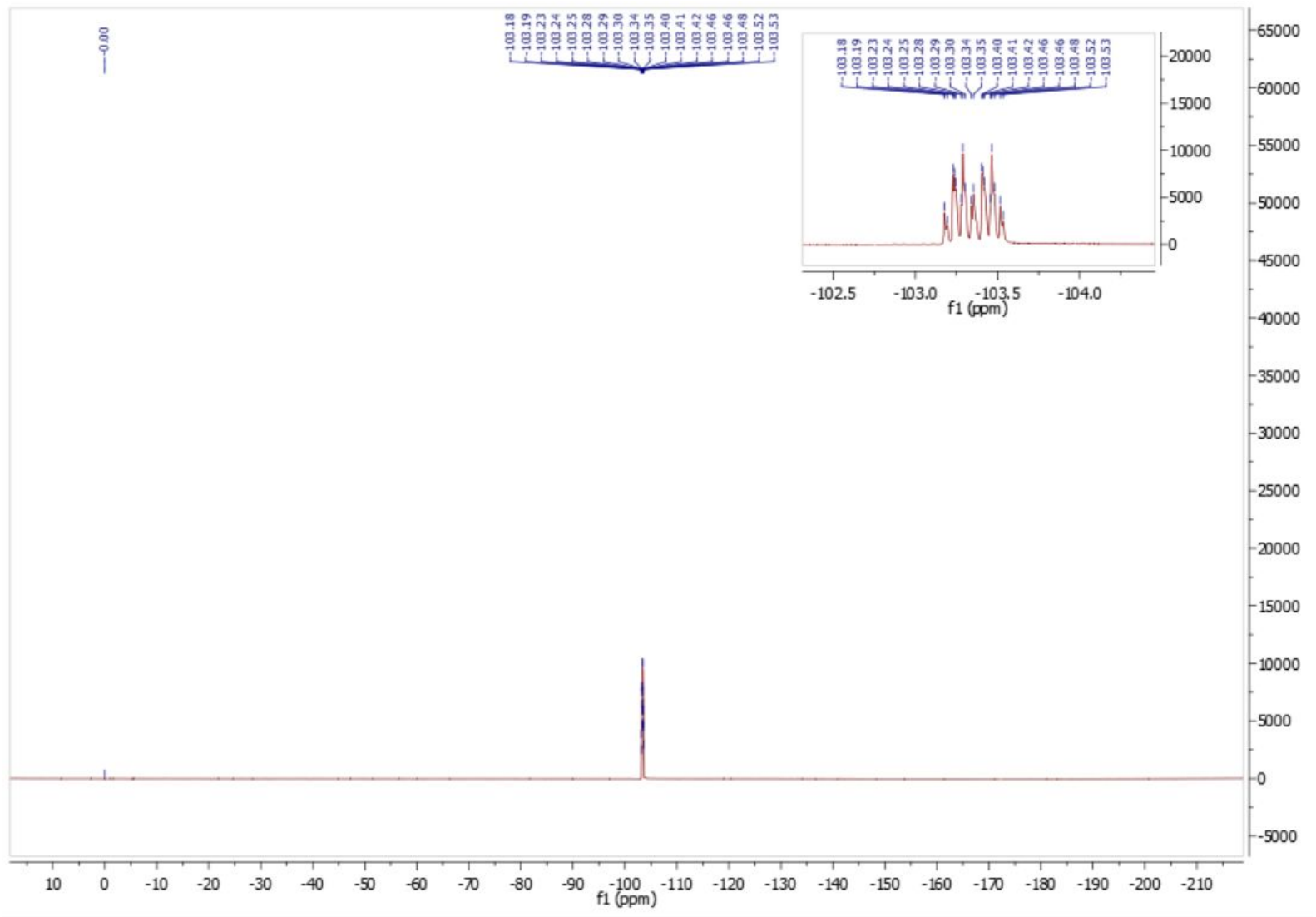


<smiles>C=C(F)CN(Cc1ccccc1)C(=O)CBr</smiles>

(2b) N-benzyl-2-bromo-N-(2-fluoroallyl)acetamide

Figure S7. ${ }^{1} \mathrm{H} \mathrm{NMR}$ spectrum of $\mathbf{2 b}\left(300 \mathrm{MHz}, \mathrm{CDCl}_{3}\right)$.

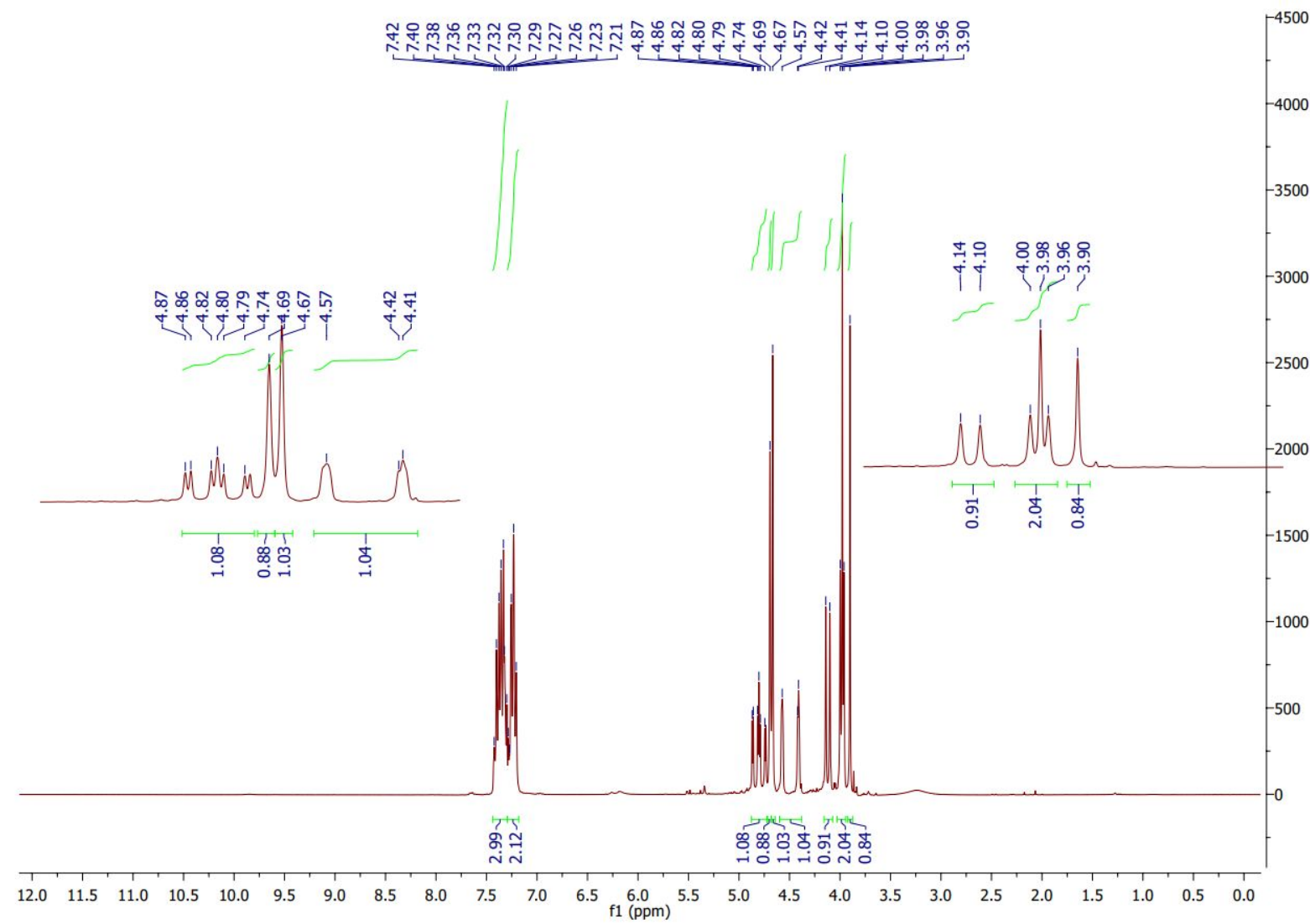


Figure S8. ${ }^{13} \mathrm{C} \mathrm{NMR}\{1 \mathrm{H}\}$ spectrum of $\mathbf{2 b}\left(75 \mathrm{MHz}, \mathrm{CDCl}_{3}\right)$.

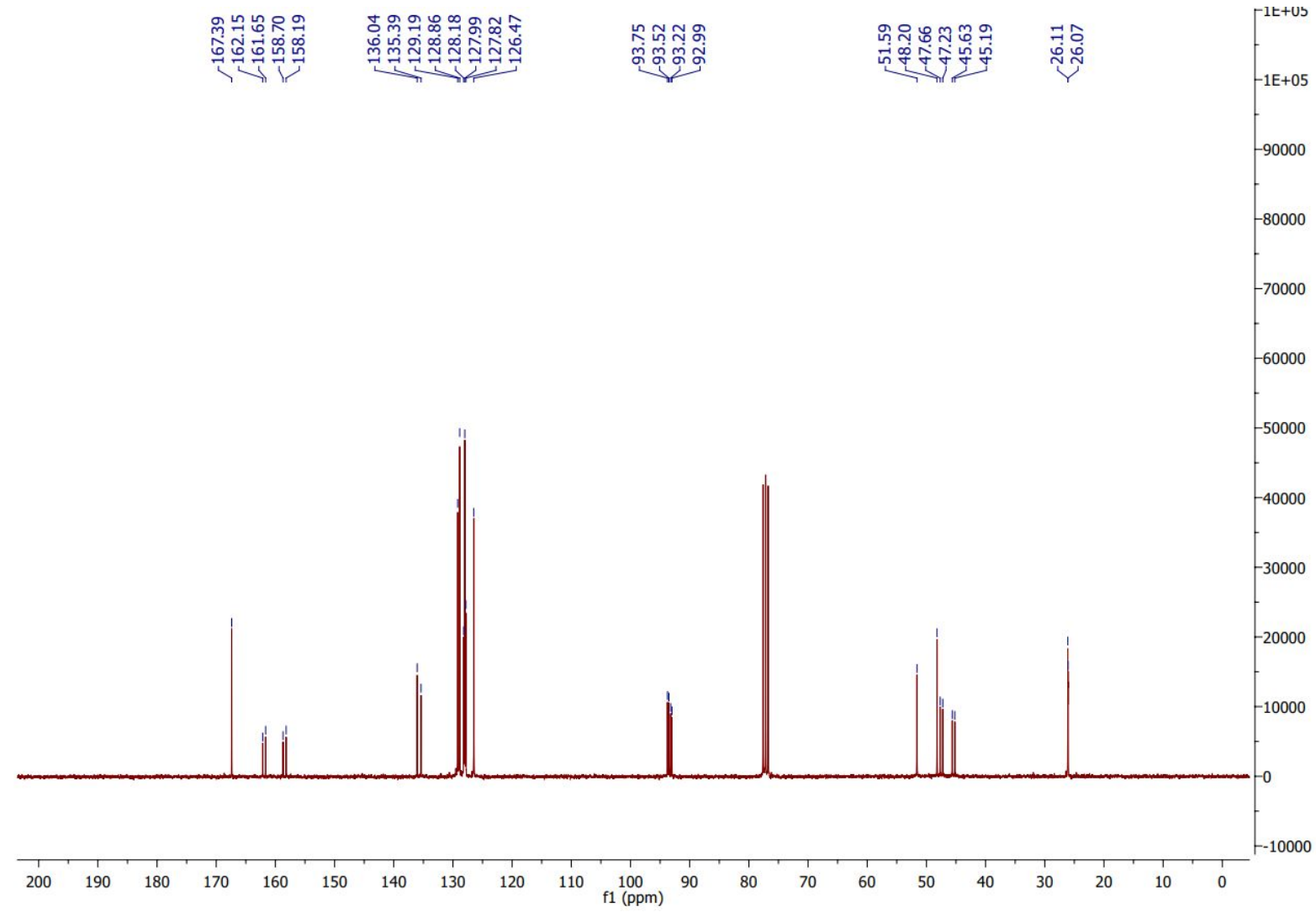

Figure S9. ${ }^{19} \mathrm{~F}$ NMR spectrum of $\mathbf{2 b}\left(282 \mathrm{MHz}, \mathrm{CDCl}_{3}\right)$.

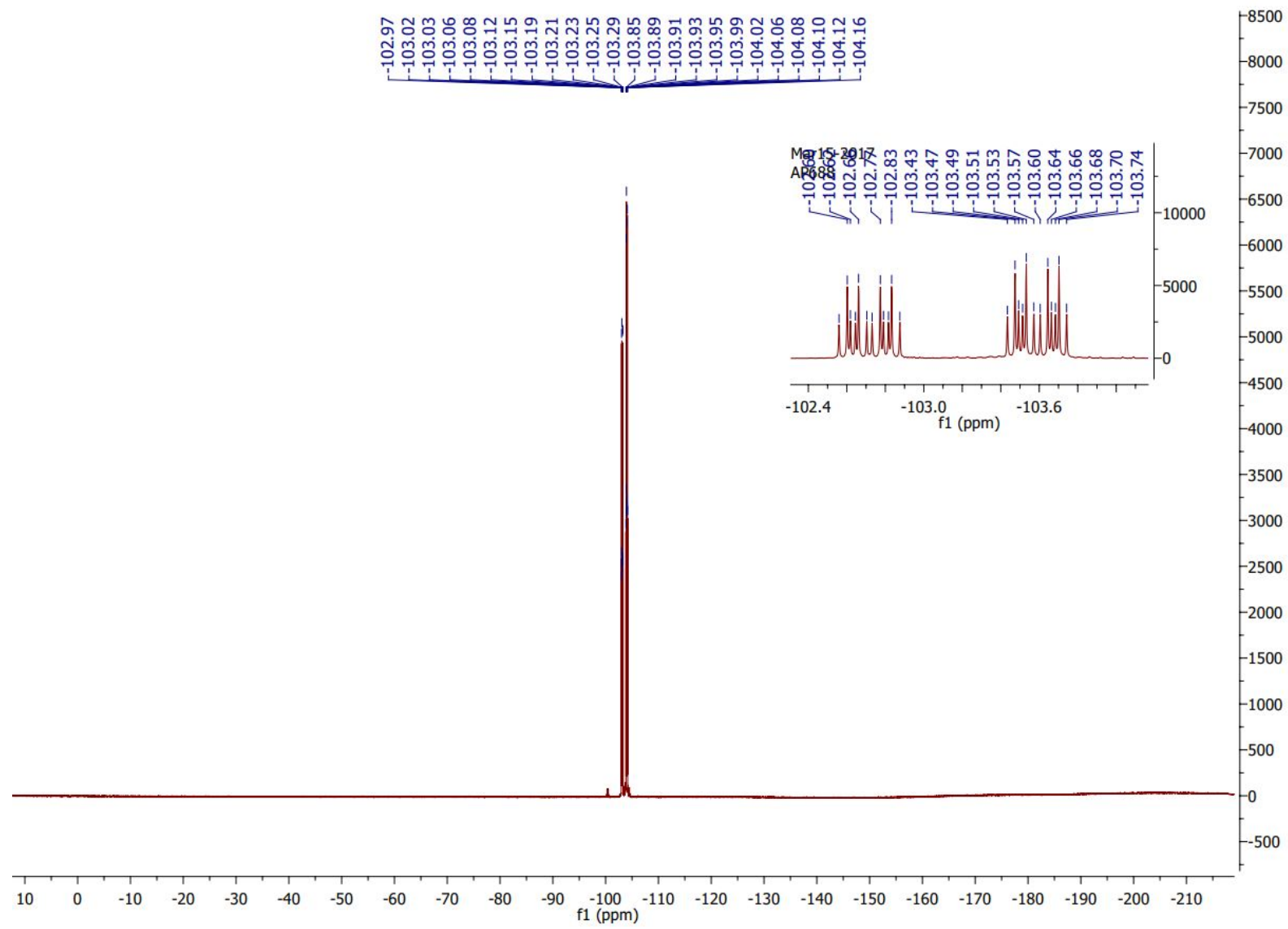


<smiles>C=C(F)CN(Cc1ccc([N+](=O)[O-])cc1)C(=O)CBr</smiles>

(2c) 2-bromo-N-(2-fluoroallyl)-N-(4-nitrobenzyl)acetamide

Figure S10. ${ }^{1} \mathrm{H}$ NMR spectrum of $2 \mathbf{c}\left(300 \mathrm{MHz}, \mathrm{CDCl}_{3}\right)$.

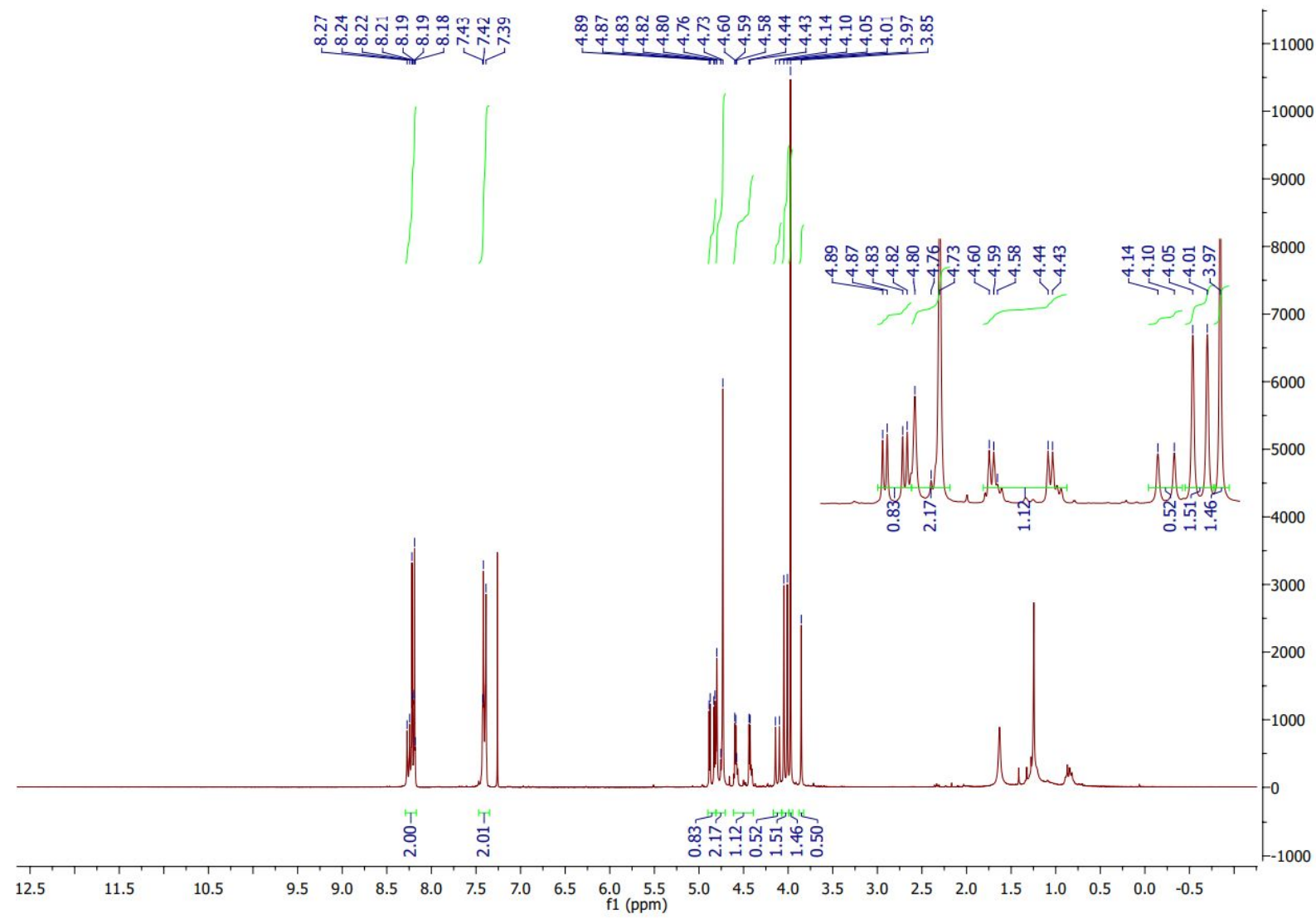


Figure S11. ${ }^{13} \mathrm{C} \mathrm{NMR}\{1 \mathrm{H}\}$ spectrum of $2 \mathrm{c}\left(75 \mathrm{MHz}, \mathrm{CDCl}_{3}\right)$.

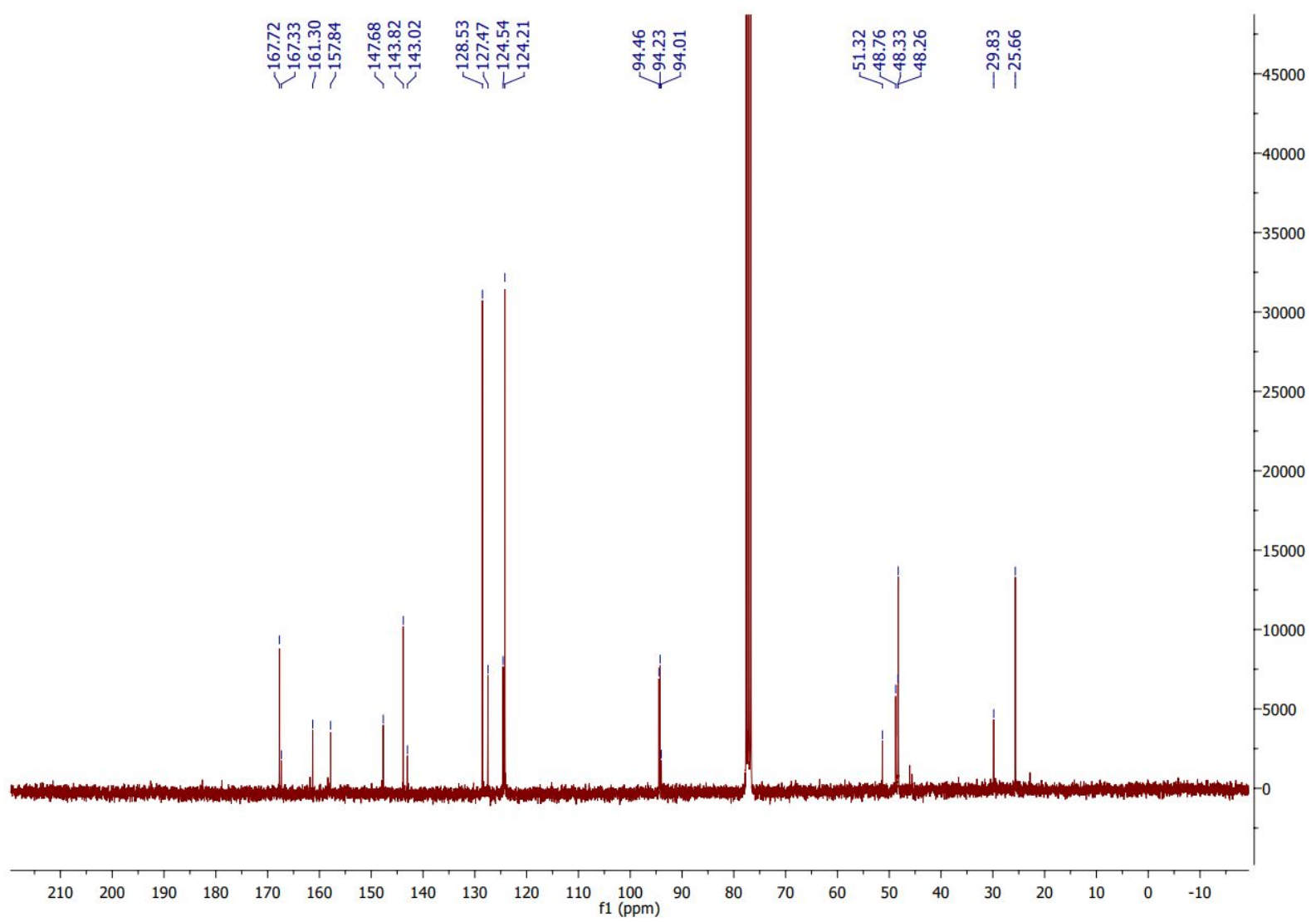

Figure S12. ${ }^{19} \mathrm{~F}$ NMR spectrum of $2 \mathbf{c}\left(282 \mathrm{MHz}, \mathrm{CDCl}_{3}\right)$.

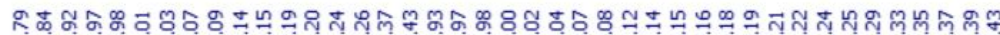

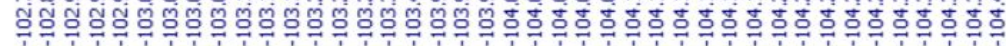

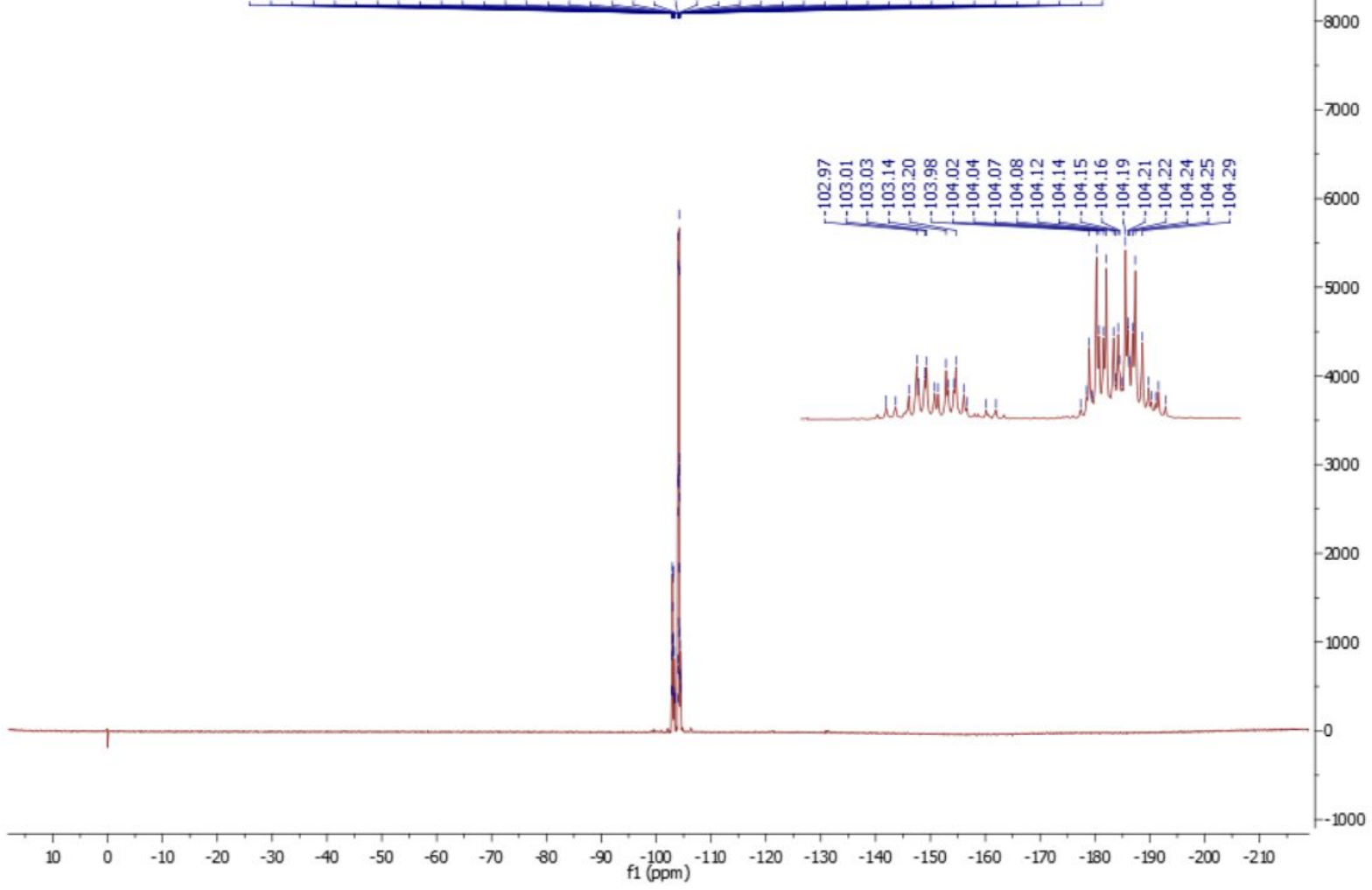


<smiles>C=C(F)CN(Cc1ccc(Cl)cc1)C(=O)CBr</smiles>

(2d) 2-bromo-N-(4-chlorobenzyl)-N-(2-fluoroallyl)acetamide

Figure S13. ${ }^{1} \mathrm{H}$ NMR spectrum of $2 \mathbf{2 d}\left(300 \mathrm{MHz}, \mathrm{CDCl}_{3}\right)$.

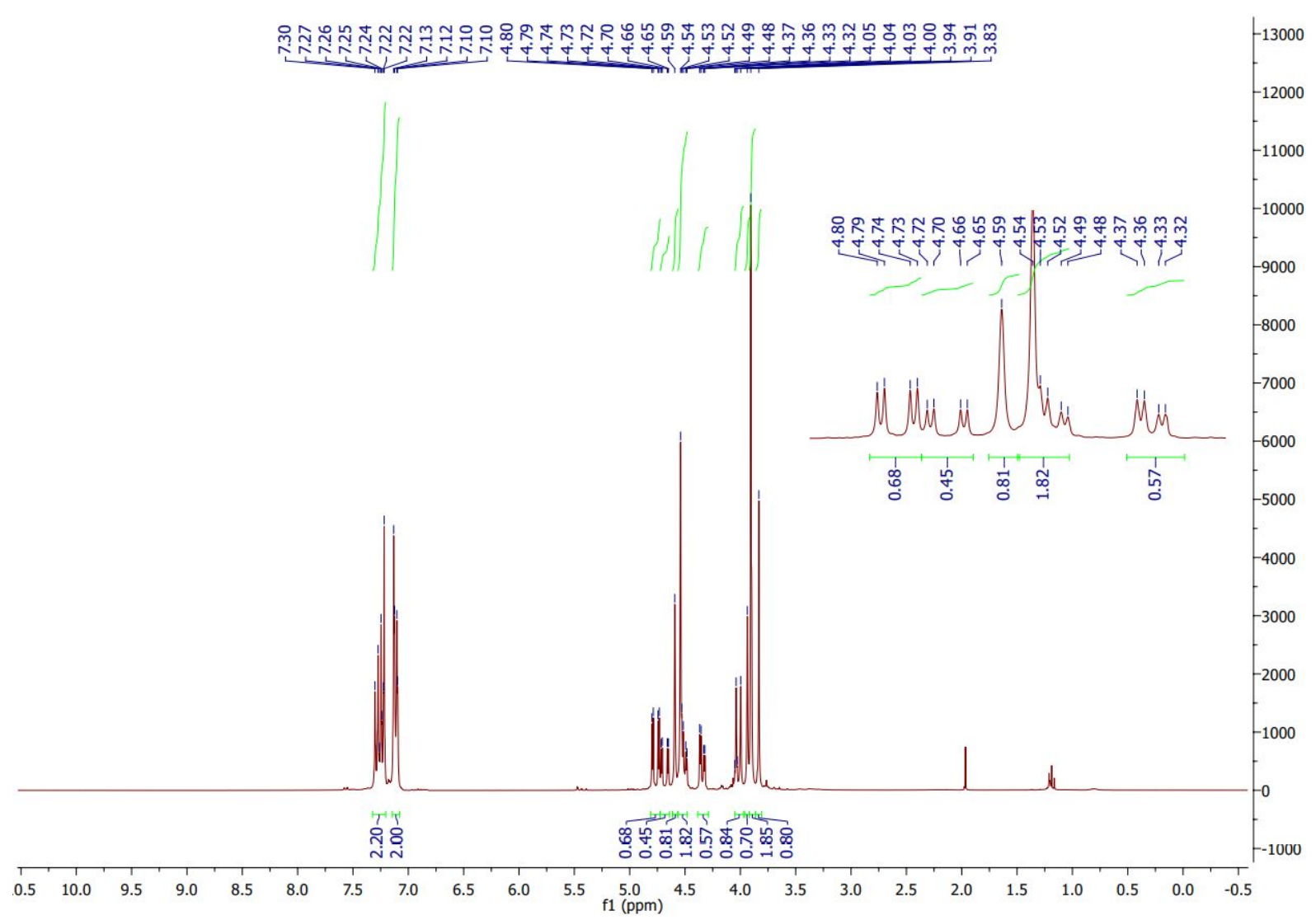


Figure S14. ${ }^{13} \mathrm{C}$ NMR $\{1 \mathrm{H}\}$ spectrum of $2 d\left(75 \mathrm{MHz}, \mathrm{CDCl}_{3}\right)$.

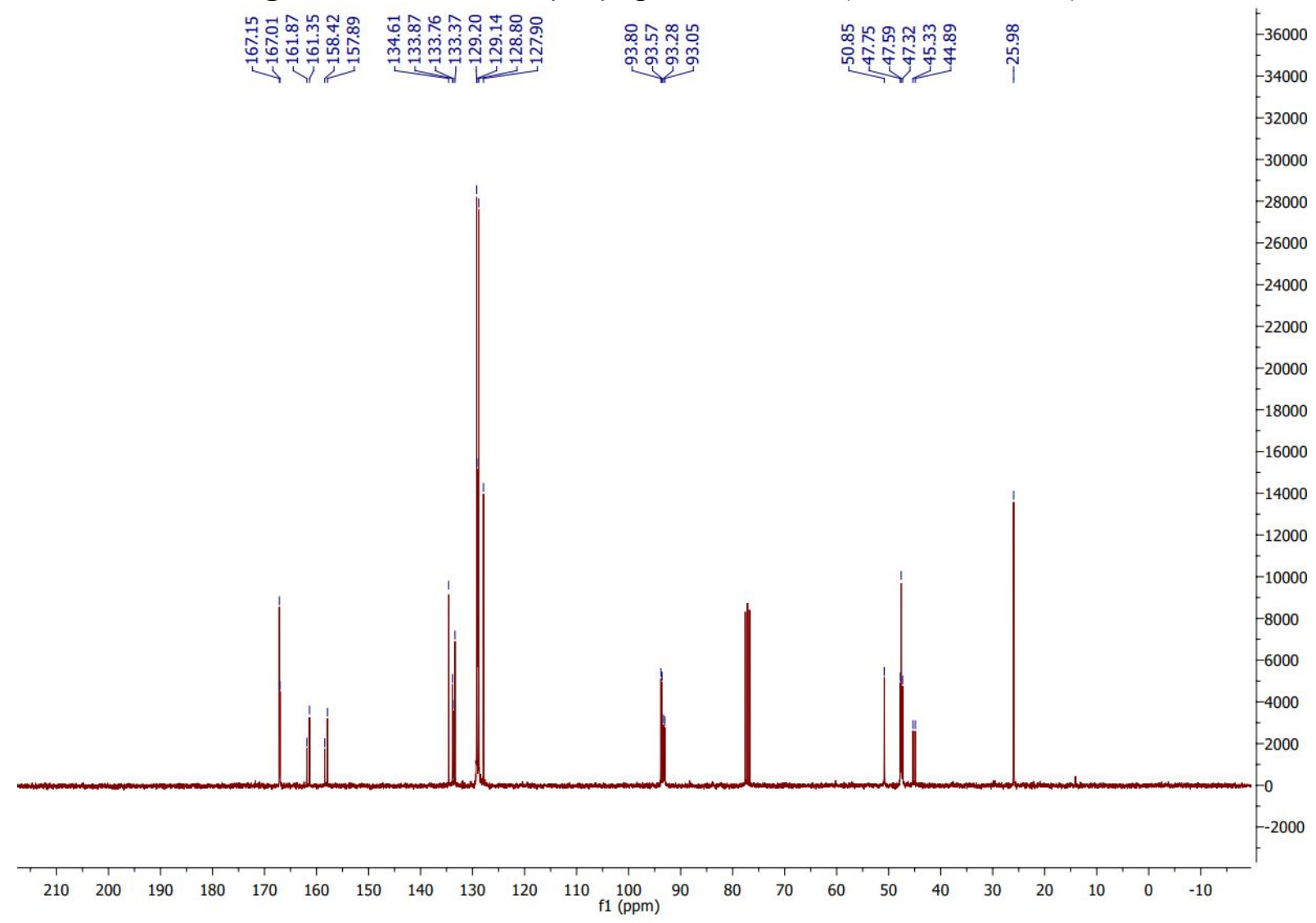

Figure S15. ${ }^{19} \mathrm{~F}$ NMR spectrum of $2 d\left(282 \mathrm{MHz}, \mathrm{CDCl}_{3}\right)$.

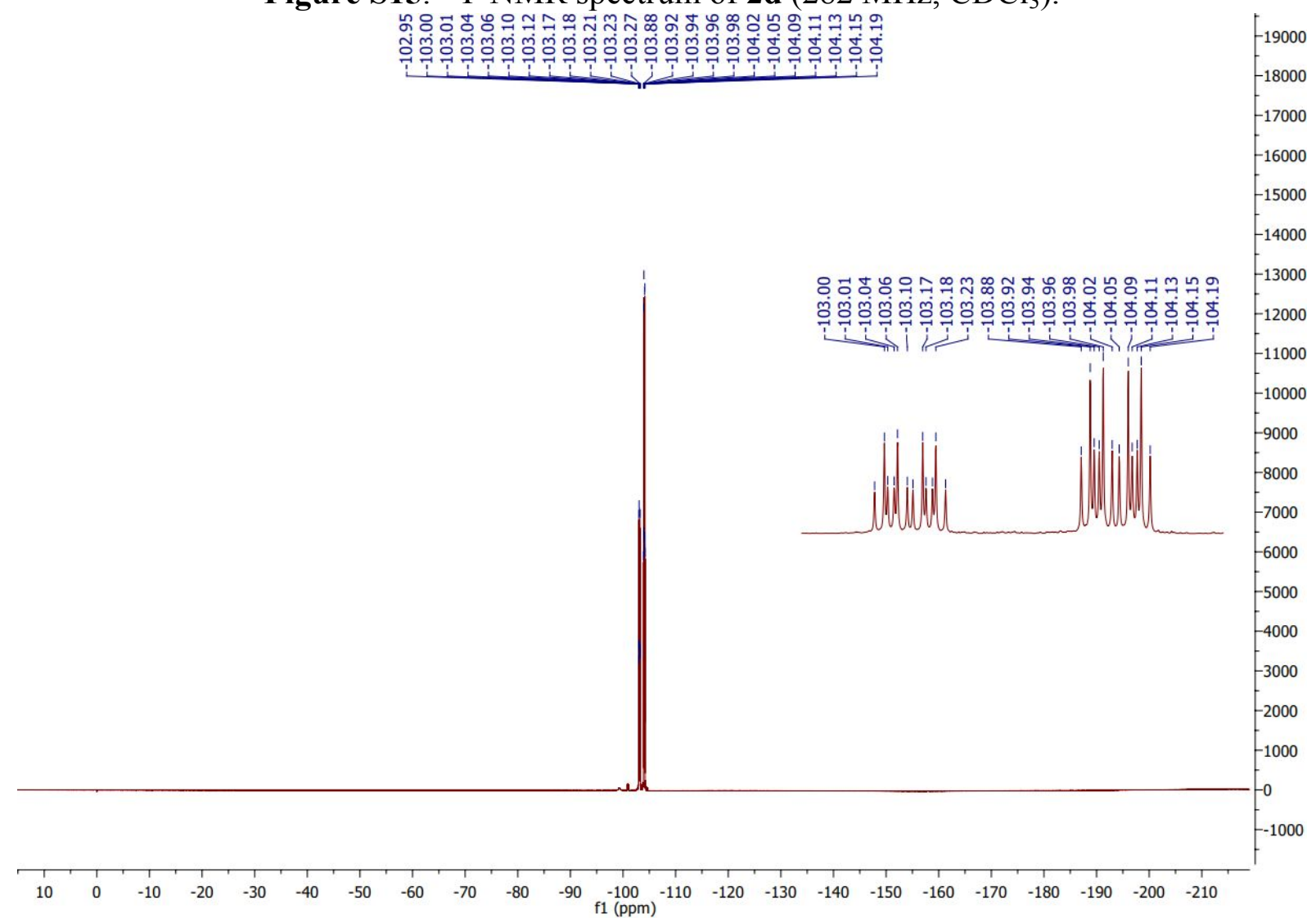


<smiles>C=C(F)CN(Cc1ccccc1)C(=O)CN</smiles>

(3b) 2-azido-N-benzyl-N-(2-fluoroallyl)acetamide

Figure S16. ${ }^{1} \mathrm{H}$ NMR spectrum of $\mathbf{3 b}\left(300 \mathrm{MHz}, \mathrm{CDCl}_{3}\right)$.

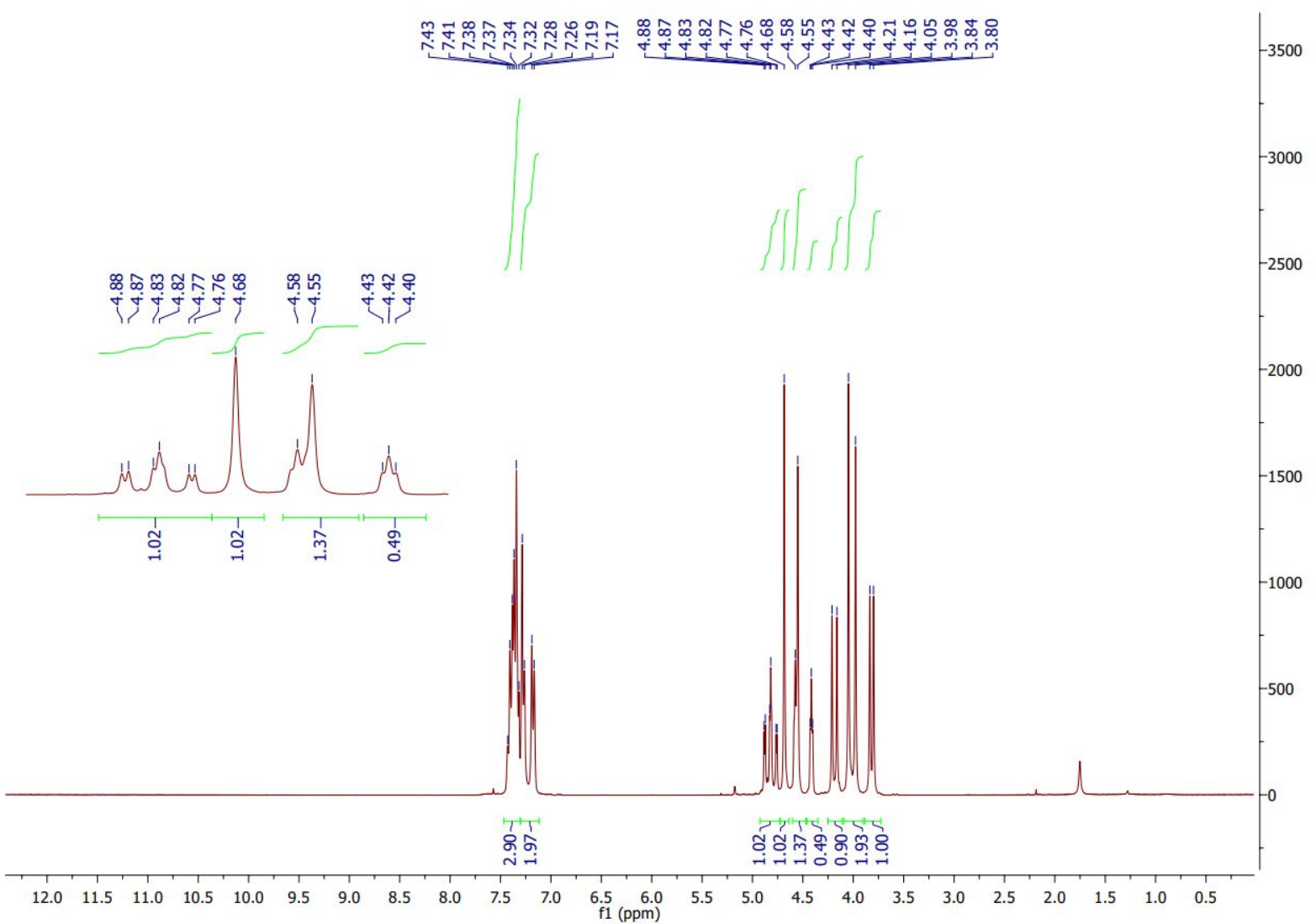


Figure S17. ${ }^{13} \mathrm{C} \mathrm{NMR}\{1 \mathrm{H}\}$ spectrum of $\mathbf{3 b}\left(75 \mathrm{MHz}, \mathrm{CDCl}_{3}\right)$.

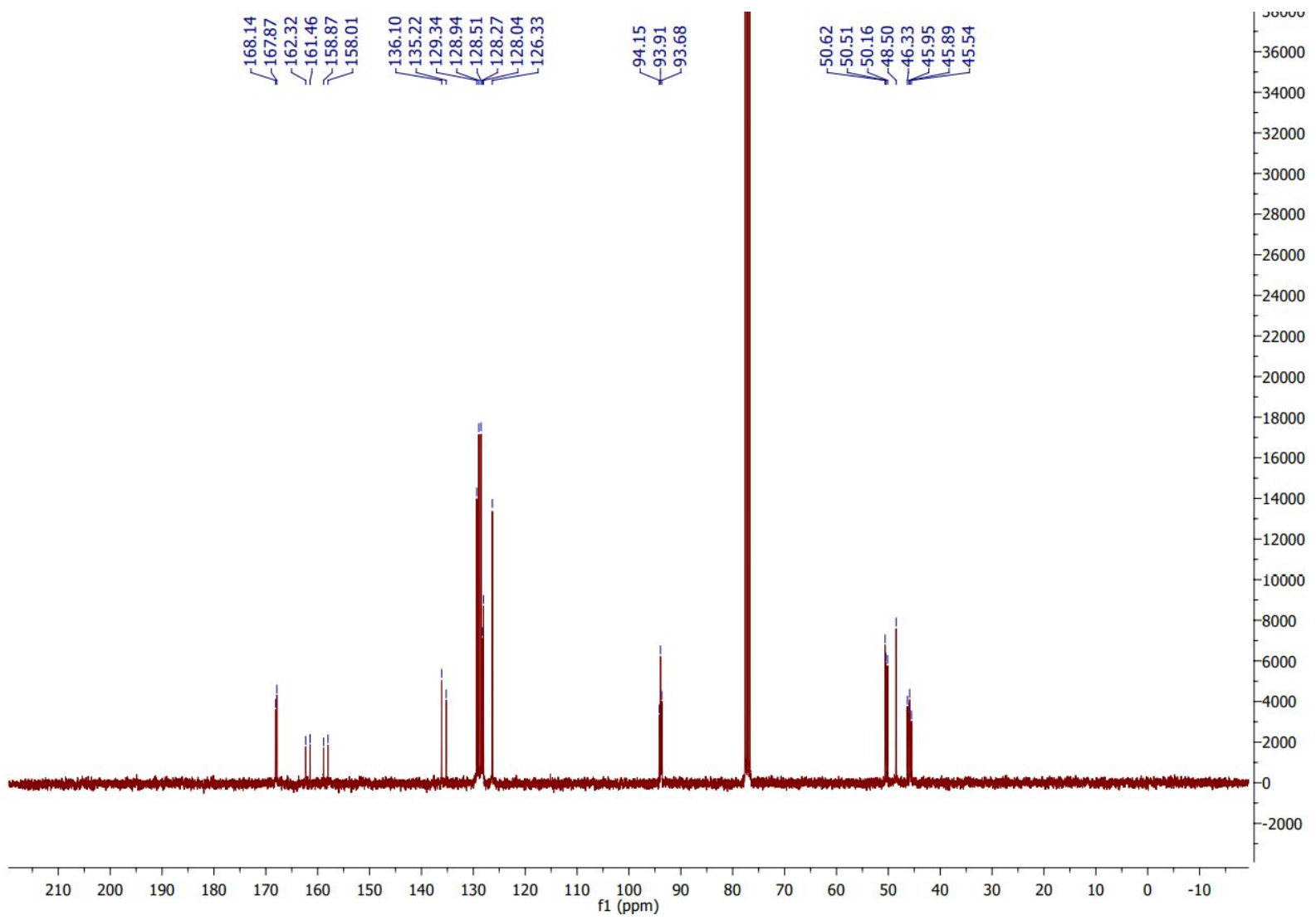

Figure S18. ${ }^{19} \mathrm{~F}$ NMR spectrum of $\mathbf{3 b}\left(282 \mathrm{MHz}, \mathrm{CDCl}_{3}\right)$.

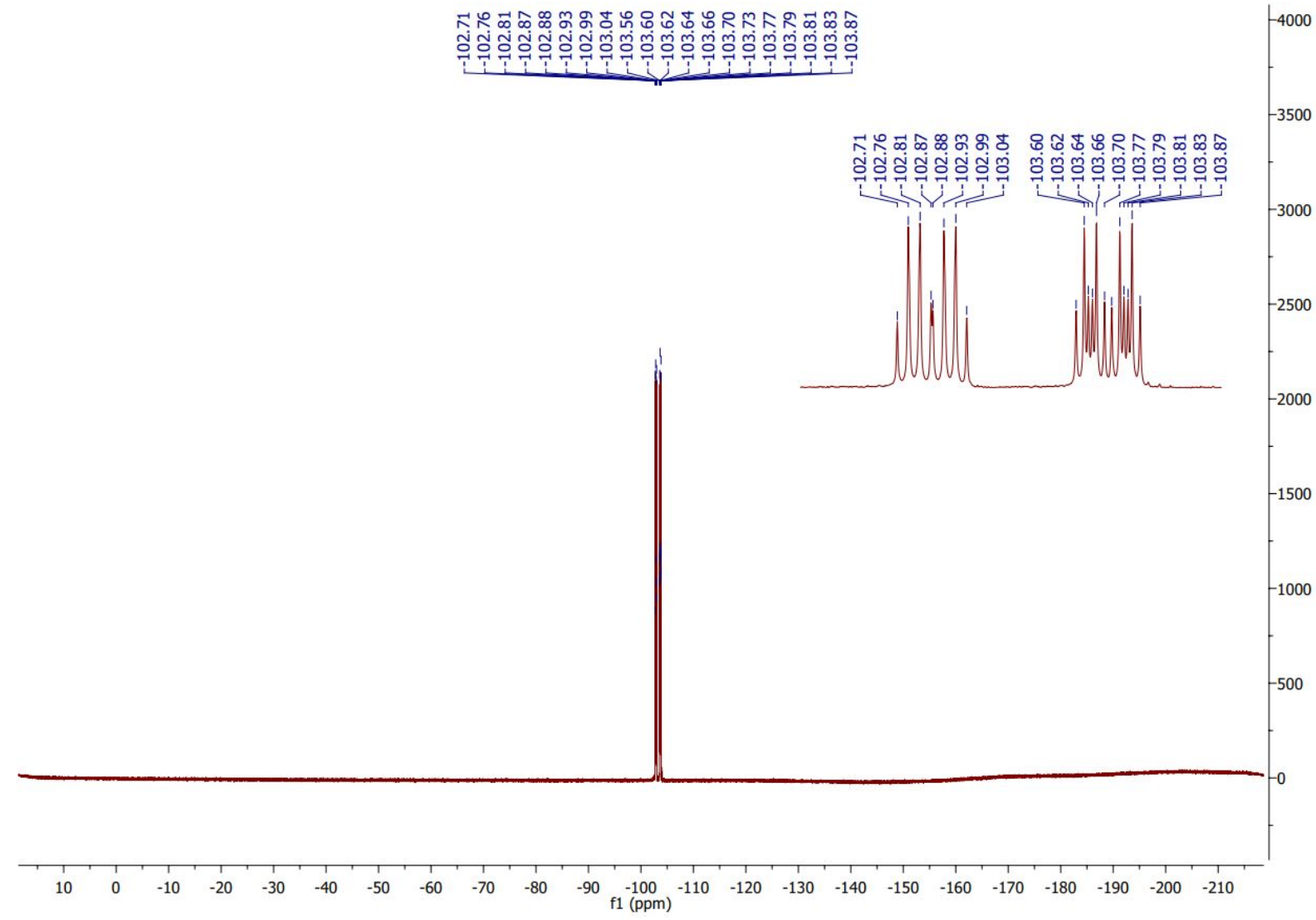


<smiles>C=C(F)CN(Cc1ccc(Cl)cc1)C(=O)CNC(=O)OC(C)(C)C</smiles>

3d di-tert-butyl (2-((2-fluoroallyl)(4-chlorobenzyl)amino)-2-oxoethyl)carbamate

Figure S19. ${ }^{1} \mathrm{H}$ NMR spectrum of $\mathbf{3 d}\left(300 \mathrm{MHz}, \mathrm{CDCl}_{3}\right)$.

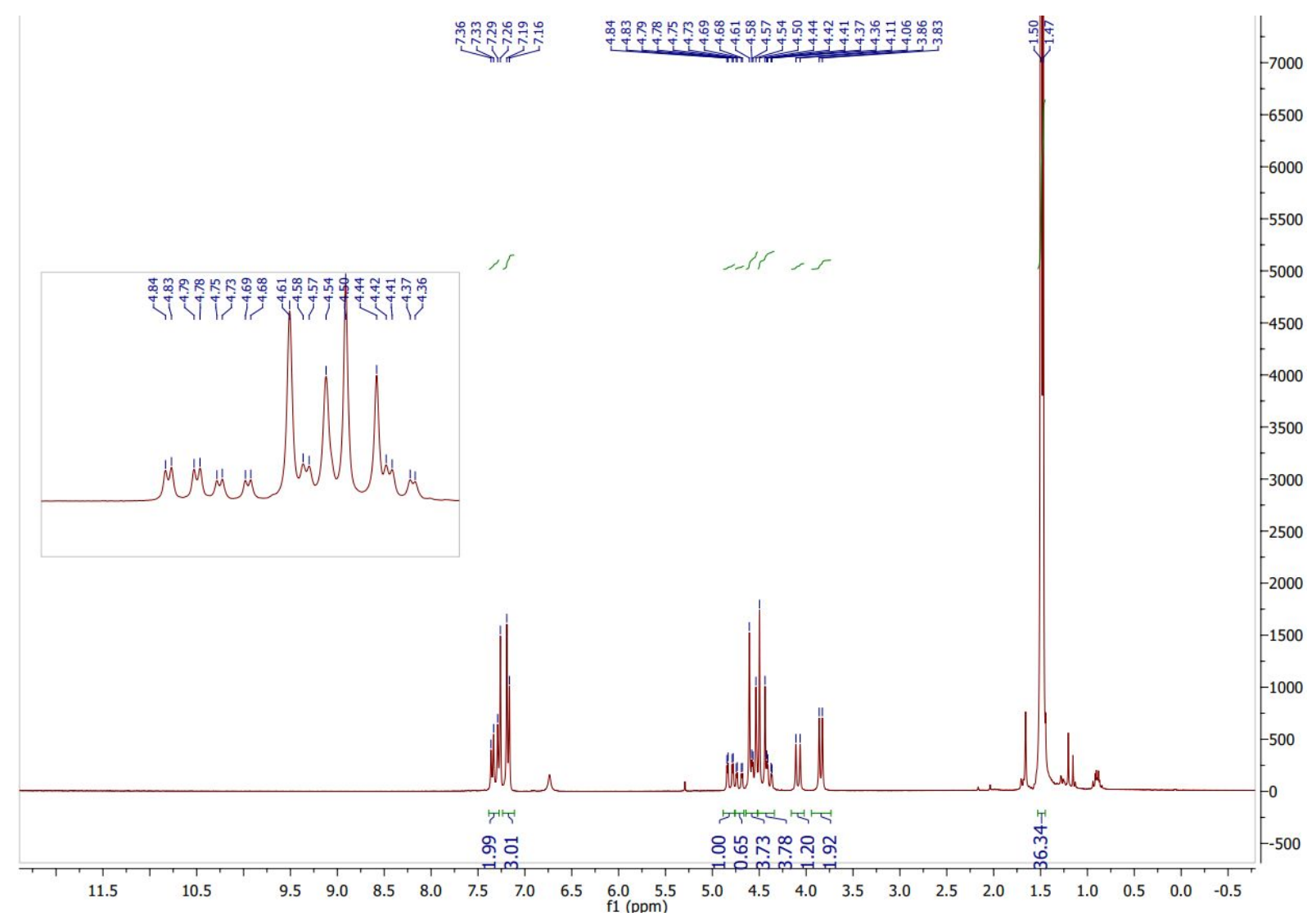


Figure S20. ${ }^{13} \mathrm{C} \mathrm{NMR}\{1 \mathrm{H}\}$ spectrum of $\mathbf{3 d}\left(75 \mathrm{MHz}, \mathrm{CDCl}_{3}\right)$.

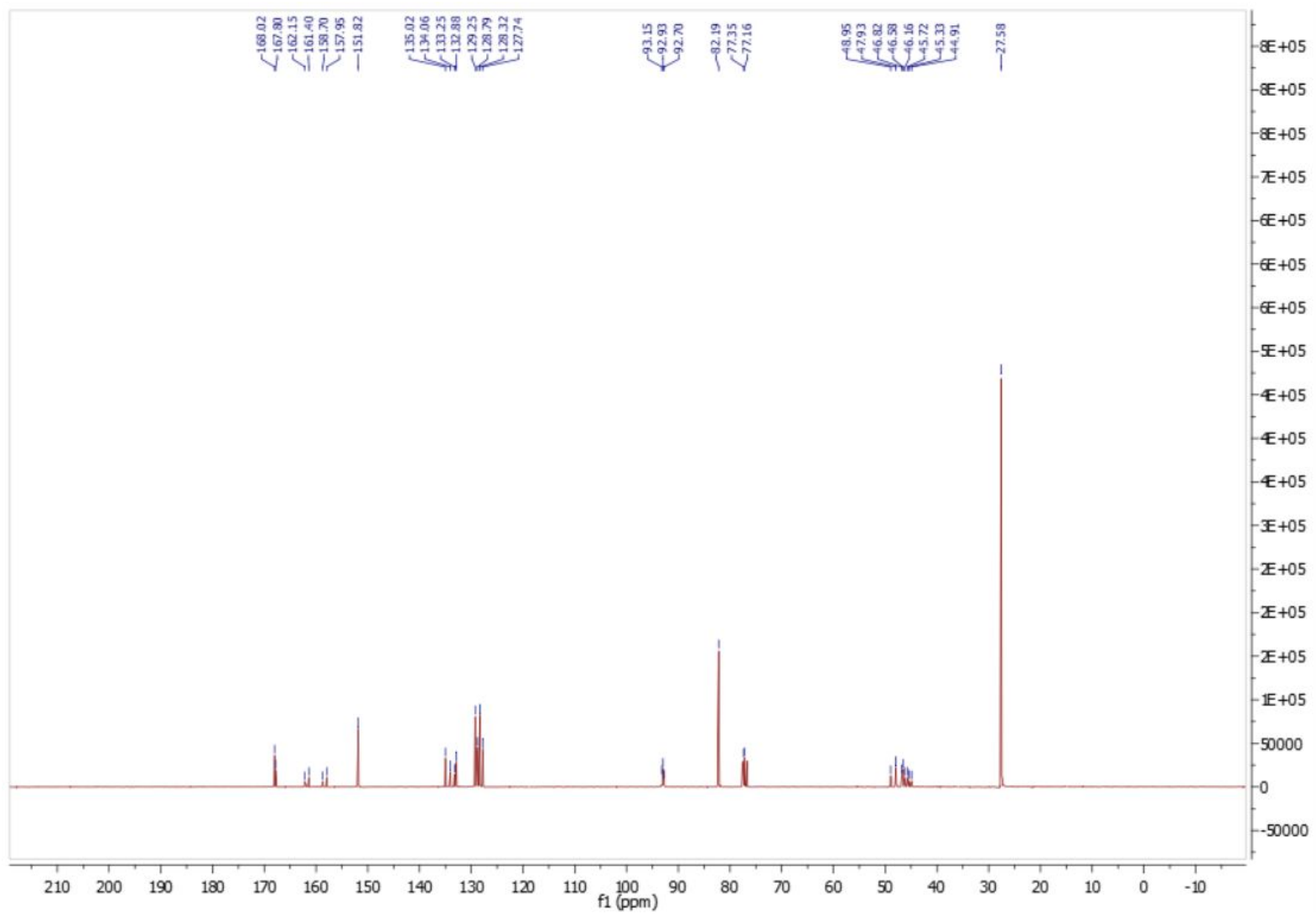

Figure S21. ${ }^{19} \mathrm{~F}$ NMR spectrum of $\mathbf{3 d}\left(282 \mathrm{MHz}, \mathrm{CDCl}_{3}\right)$.

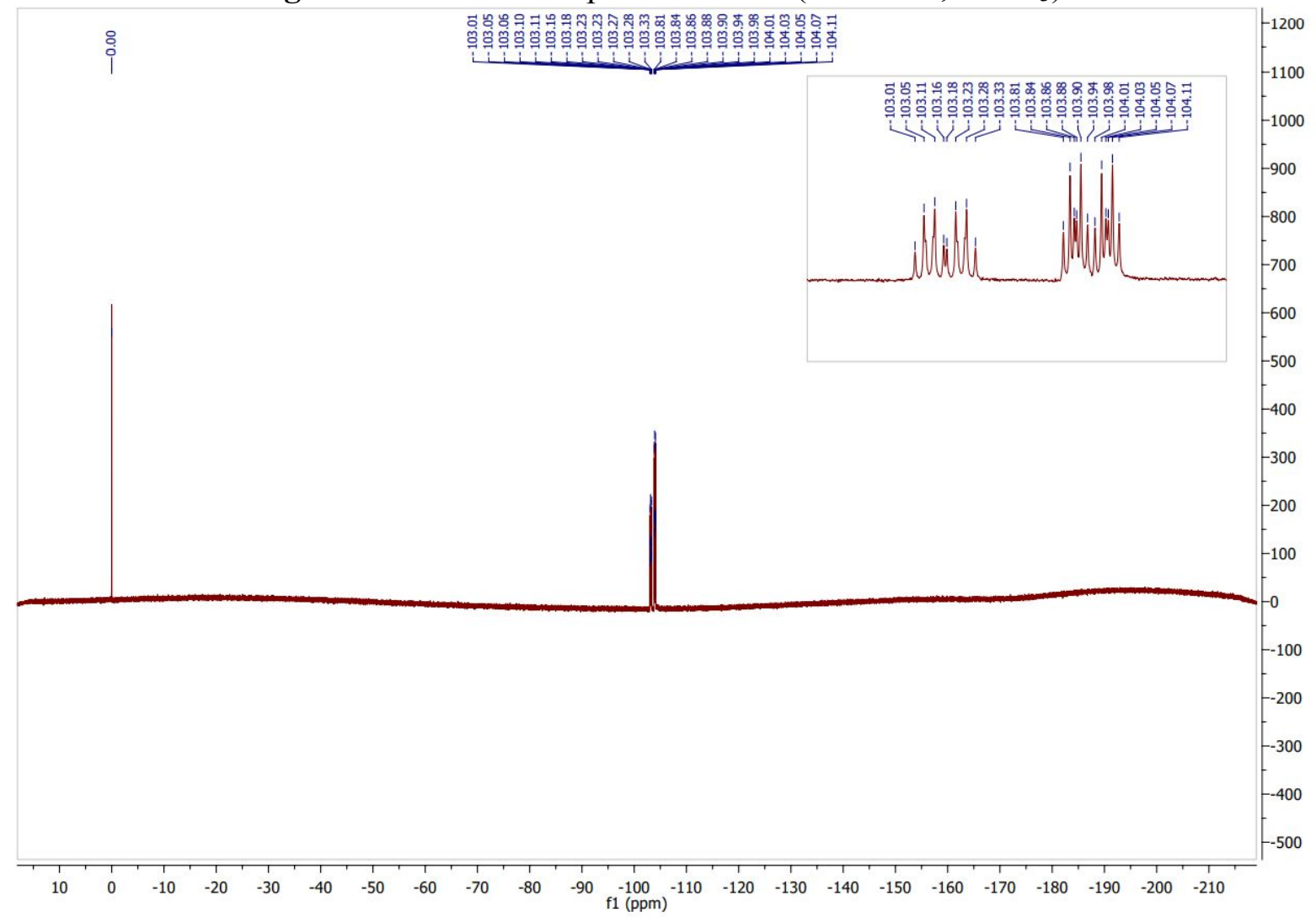


<smiles>C=C(F)CN(Cc1ccccc1)C(=O)CN</smiles>

(4b) 2-amino-N-benzyl-N-(2-fluoroallyl)acetamide

Figure S22. ${ }^{1} \mathrm{H}$ NMR spectrum of $\mathbf{4 b}\left(300 \mathrm{MHz}, \mathrm{CDCl}_{3}\right)$.

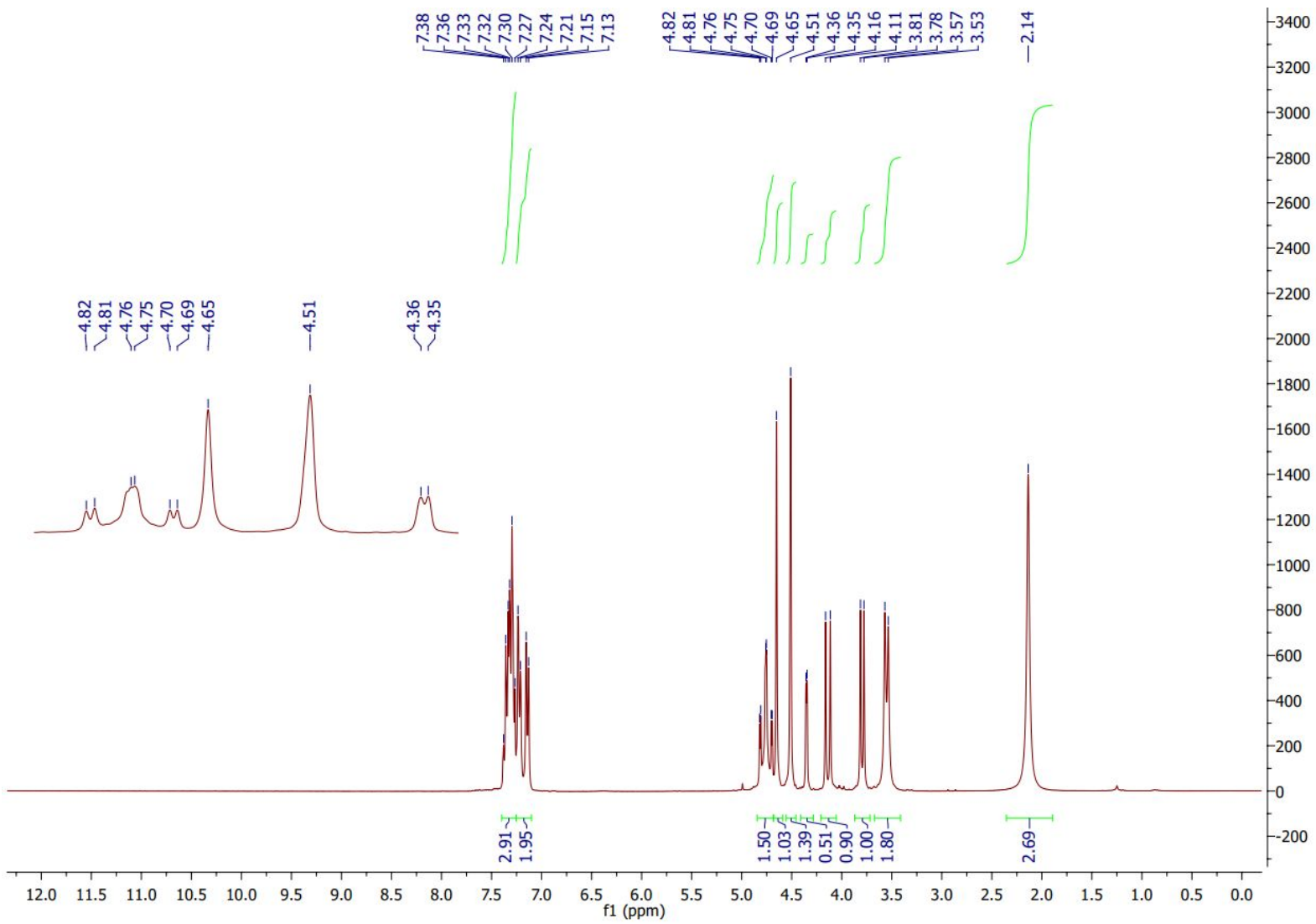


Figure S23. ${ }^{13} \mathrm{C} \mathrm{NMR}\{1 \mathrm{H}\}$ spectrum of $\mathbf{4 b}\left(75 \mathrm{MHz}, \mathrm{CDCl}_{3}\right)$.

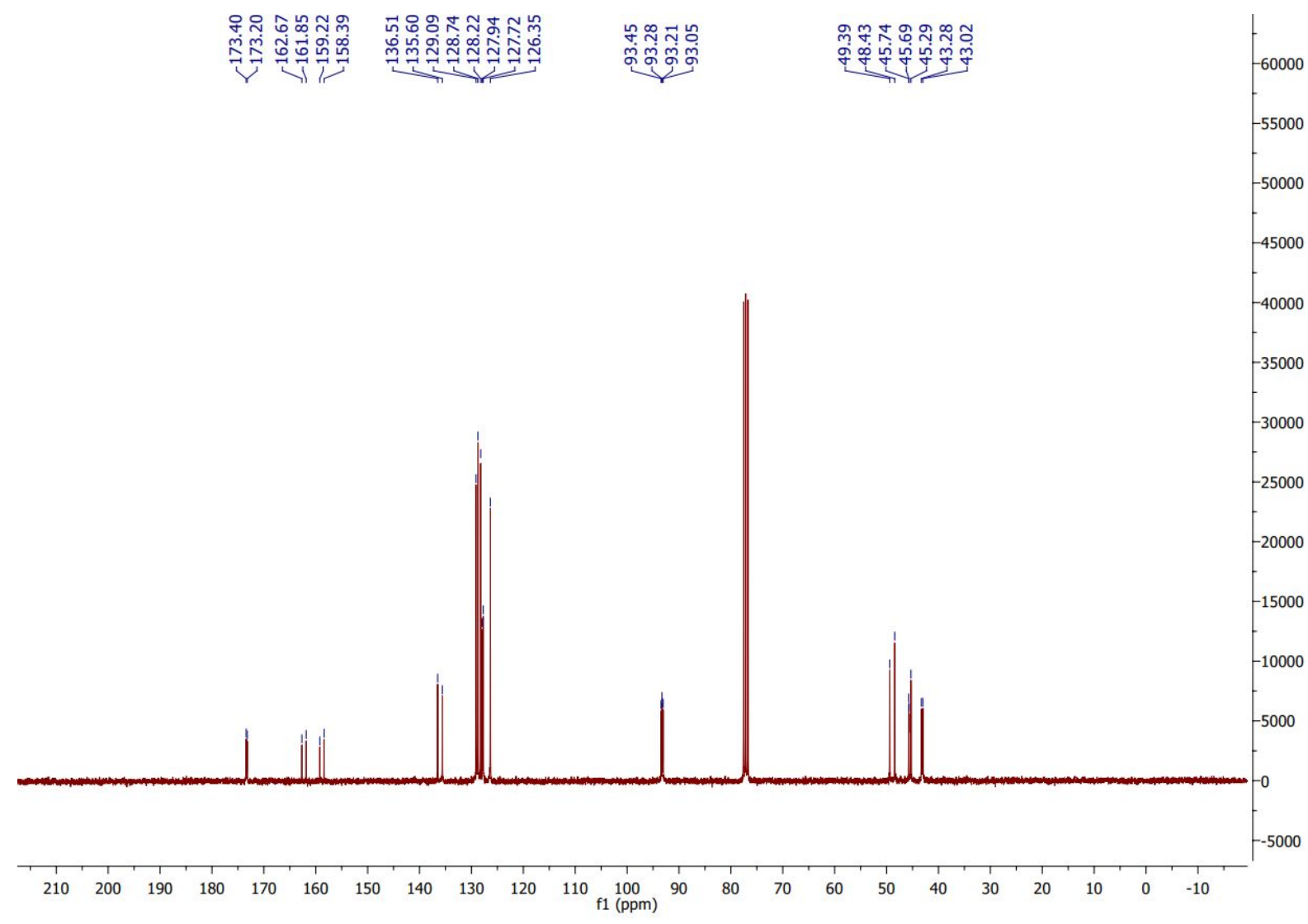

Figure S24. ${ }^{19} \mathrm{~F}$ NMR spectrum of $\mathbf{4 b}\left(282 \mathrm{MHz}, \mathrm{CDCl}_{3}\right)$.

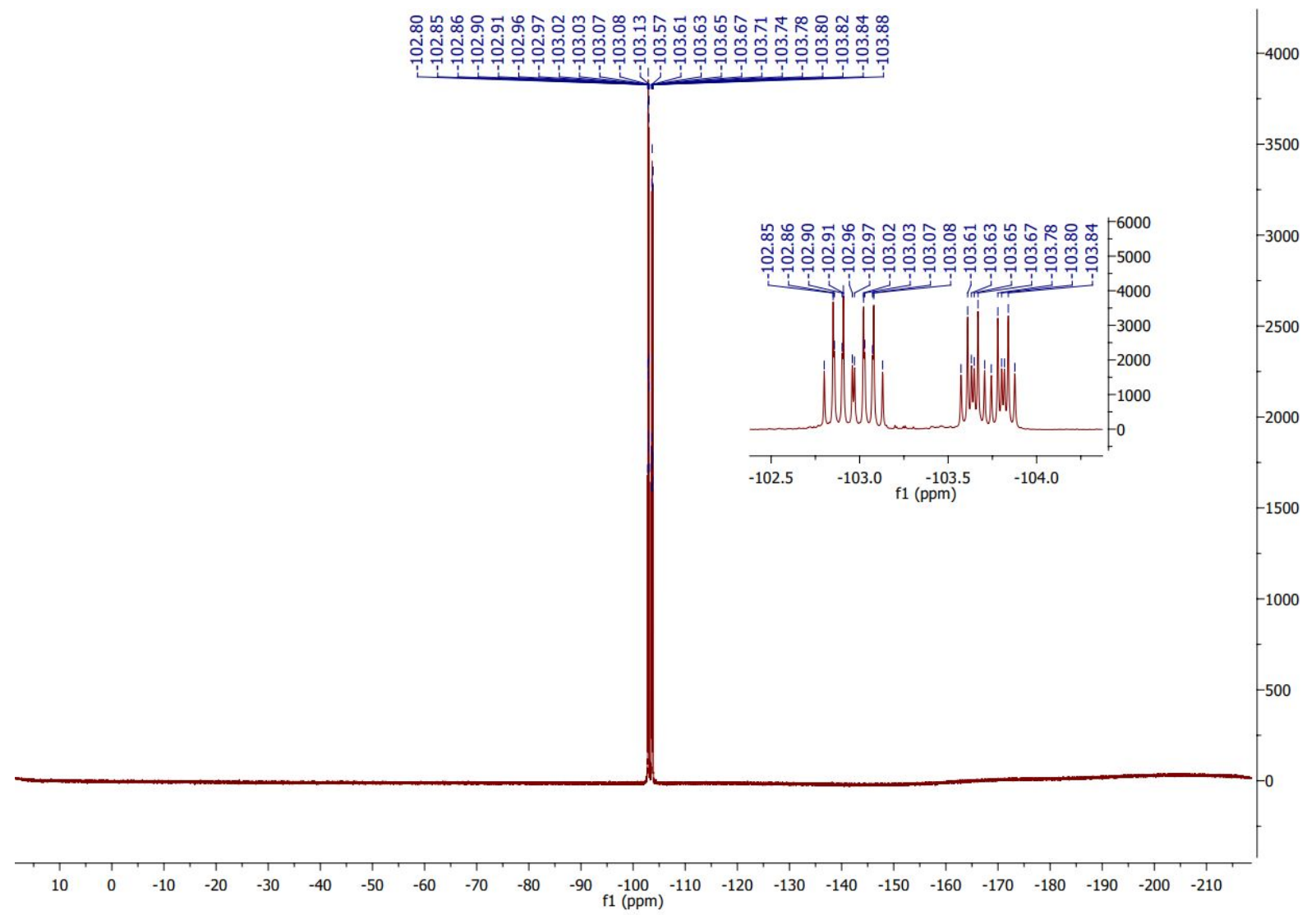


<smiles>C=C(F)CN(Cc1ccc([N+](=O)[O-])cc1)C(=O)CNC(=O)[O-]</smiles>

(3c) di-tert-butyl (2-((2-fluoroallyl)(4-nitrobenzyl)amino)-2-oxoethyl)carbamate

Figure S25. ${ }^{1} \mathrm{H}$ NMR spectrum of $\mathbf{3 c}\left(300 \mathrm{MHz}, \mathrm{CDCl}_{3}\right)$.

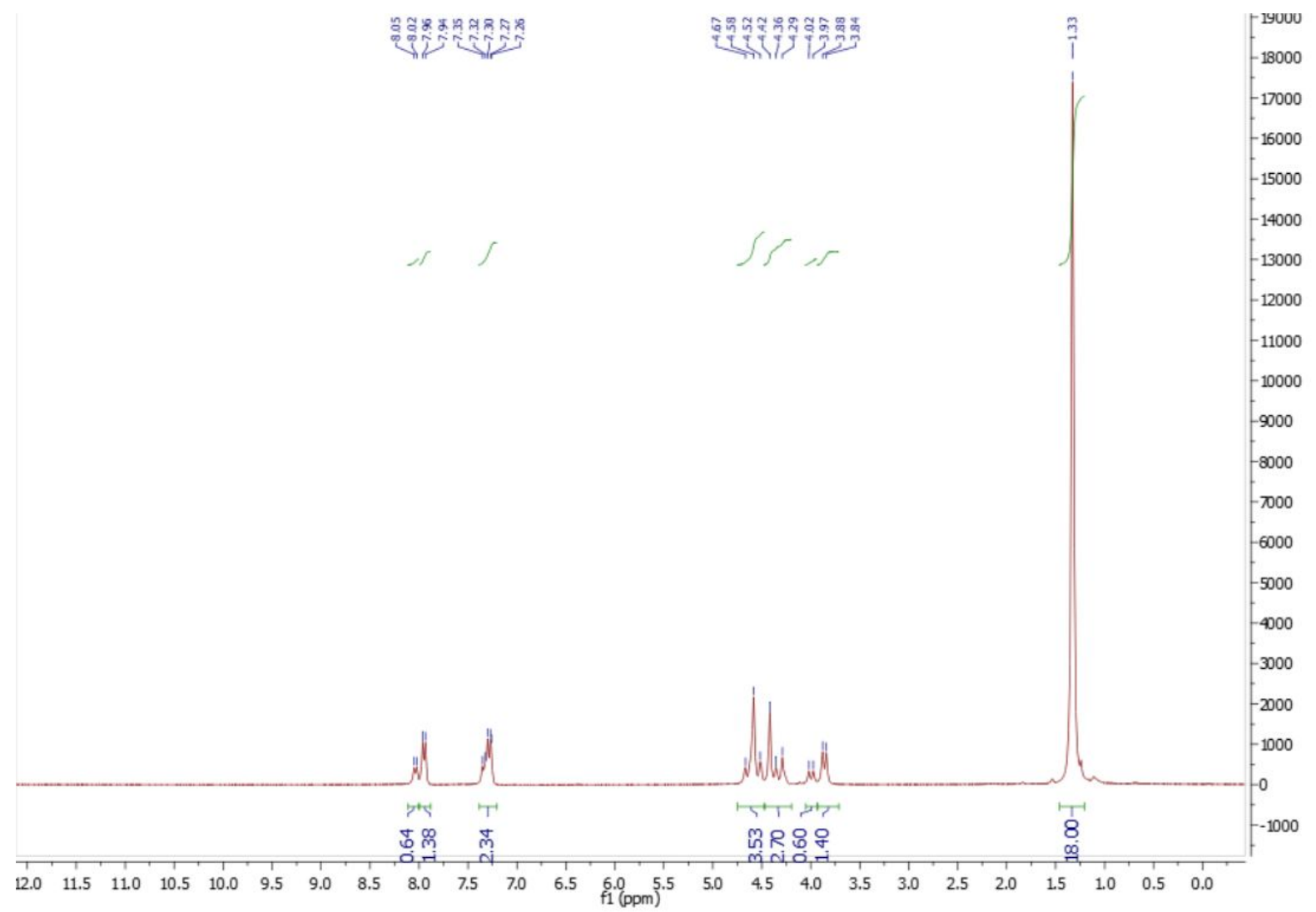


Figure S26. ${ }^{13} \mathrm{C} \mathrm{NMR}\{1 \mathrm{H}\}$ spectrum of $\mathbf{3 c}\left(75 \mathrm{MHz}, \mathrm{CDCl}_{3}\right)$.

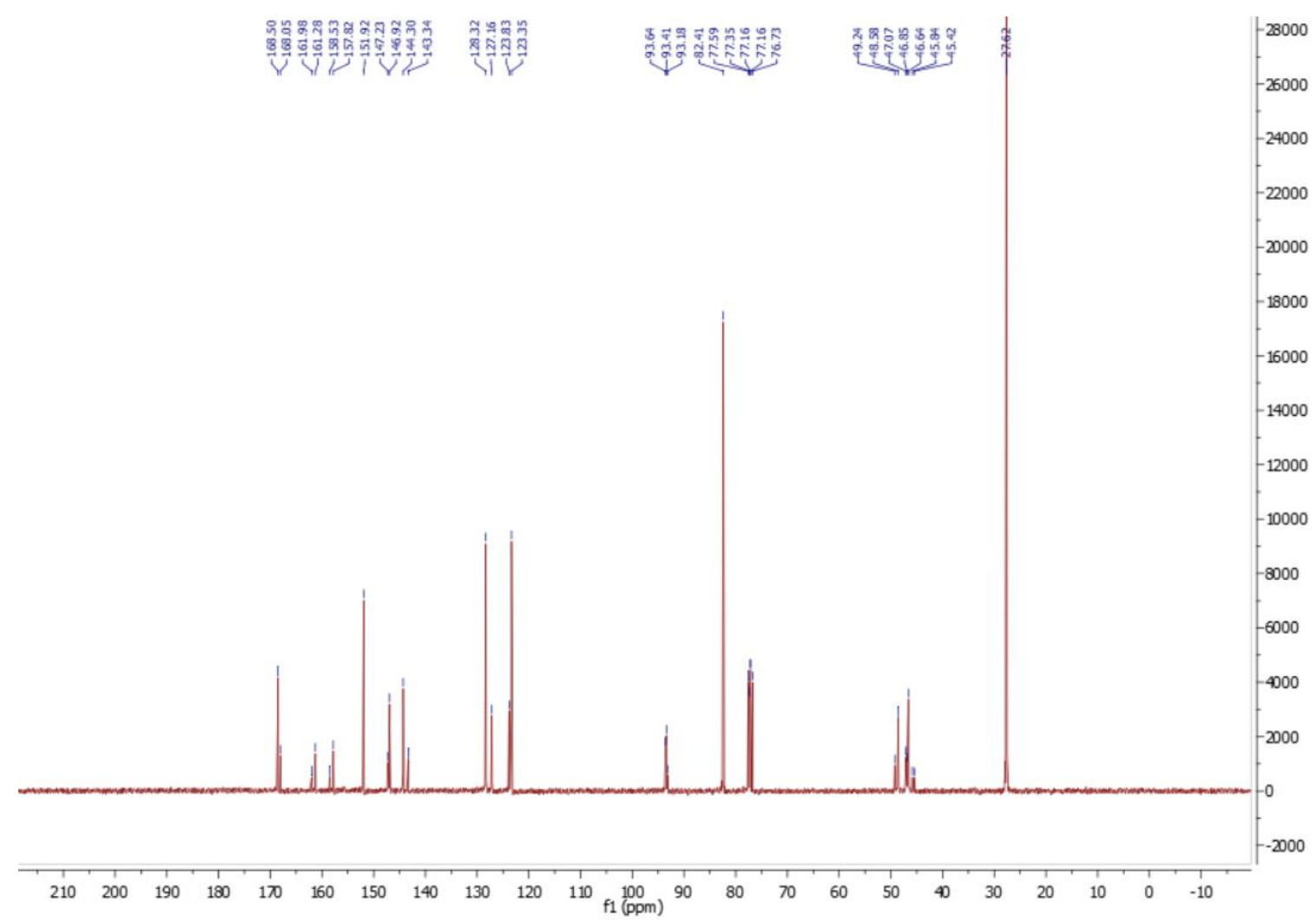

Figure S27. ${ }^{19} \mathrm{~F}$ NMR spectrum of $\mathbf{3 c}\left(282 \mathrm{MHz}, \mathrm{CDCl}_{3}\right)$.

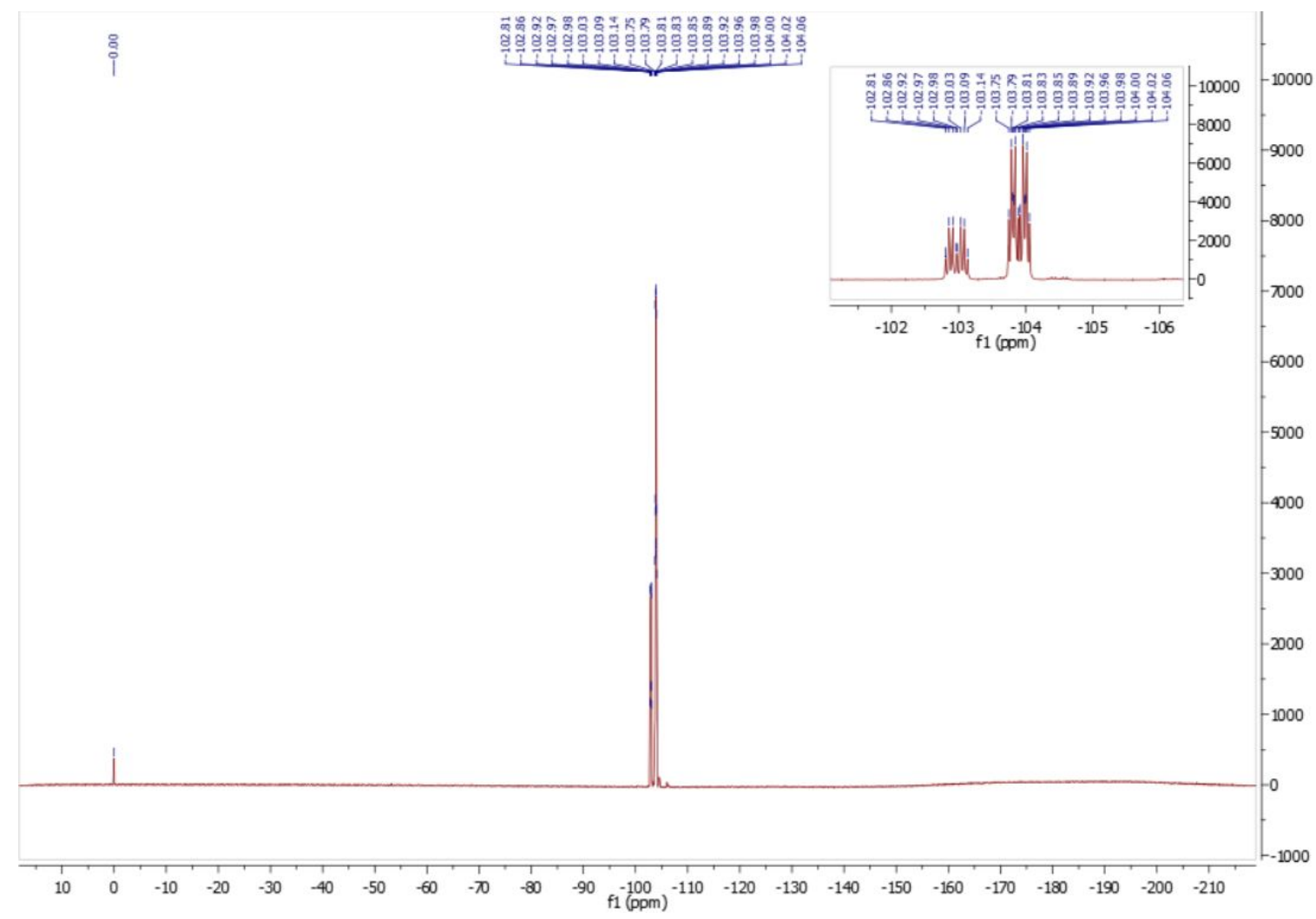


<smiles>C=C(F)CN(Cc1ccccc1)C(=O)C=N</smiles>

(5b) N-benzyl-N-(2-fluoroallyl)diazoacetamide

Figure S28. ${ }^{1} \mathrm{H}$ NMR spectrum of $\mathbf{5 b}\left(300 \mathrm{MHz}, \mathrm{CDCl}_{3}\right)$.

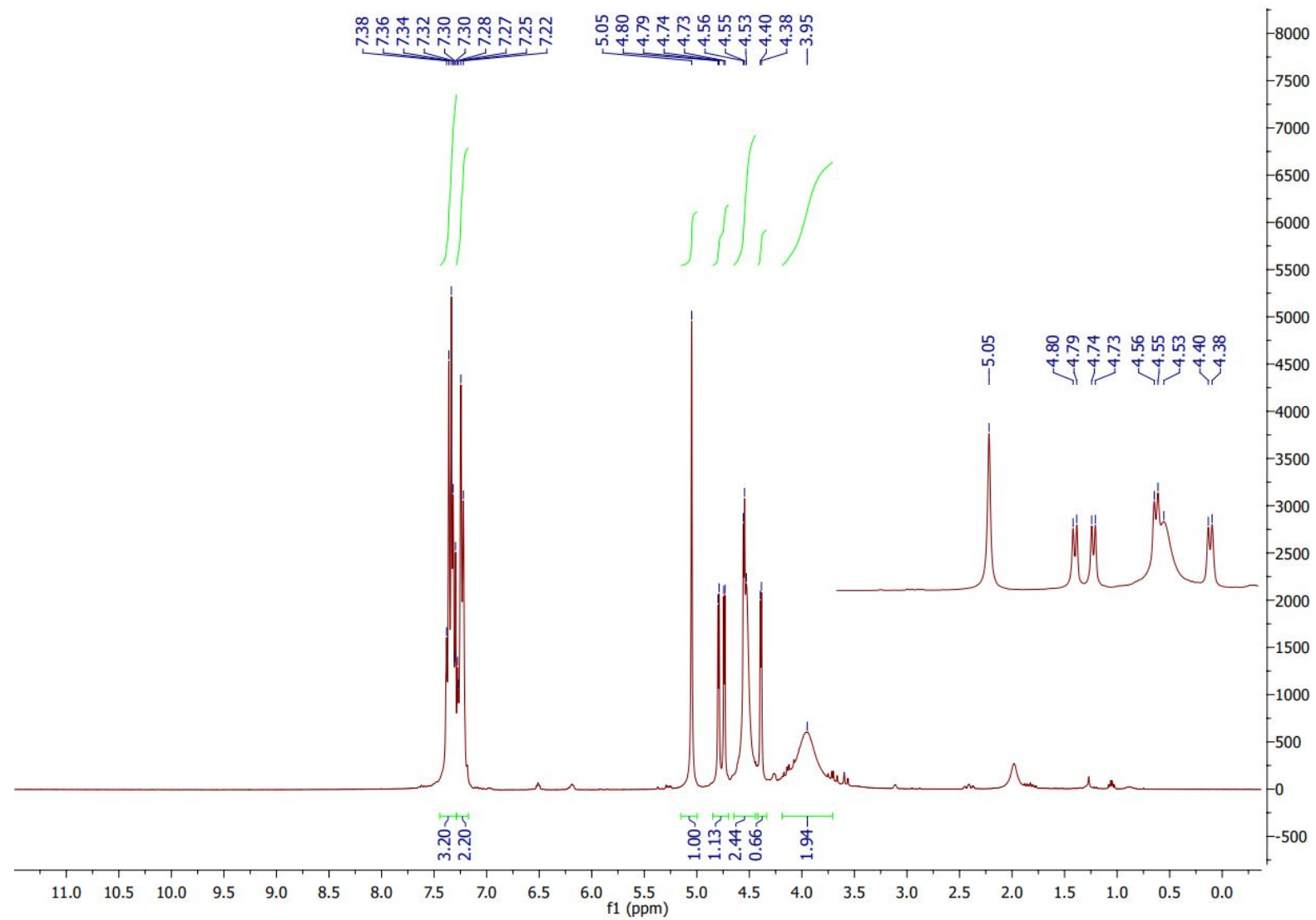


Figure S29. ${ }^{13} \mathrm{C} \mathrm{NMR}\{1 \mathrm{H}\}$ spectrum of $\mathbf{5 b}\left(75 \mathrm{MHz}, \mathrm{CDCl}_{3}\right)$.

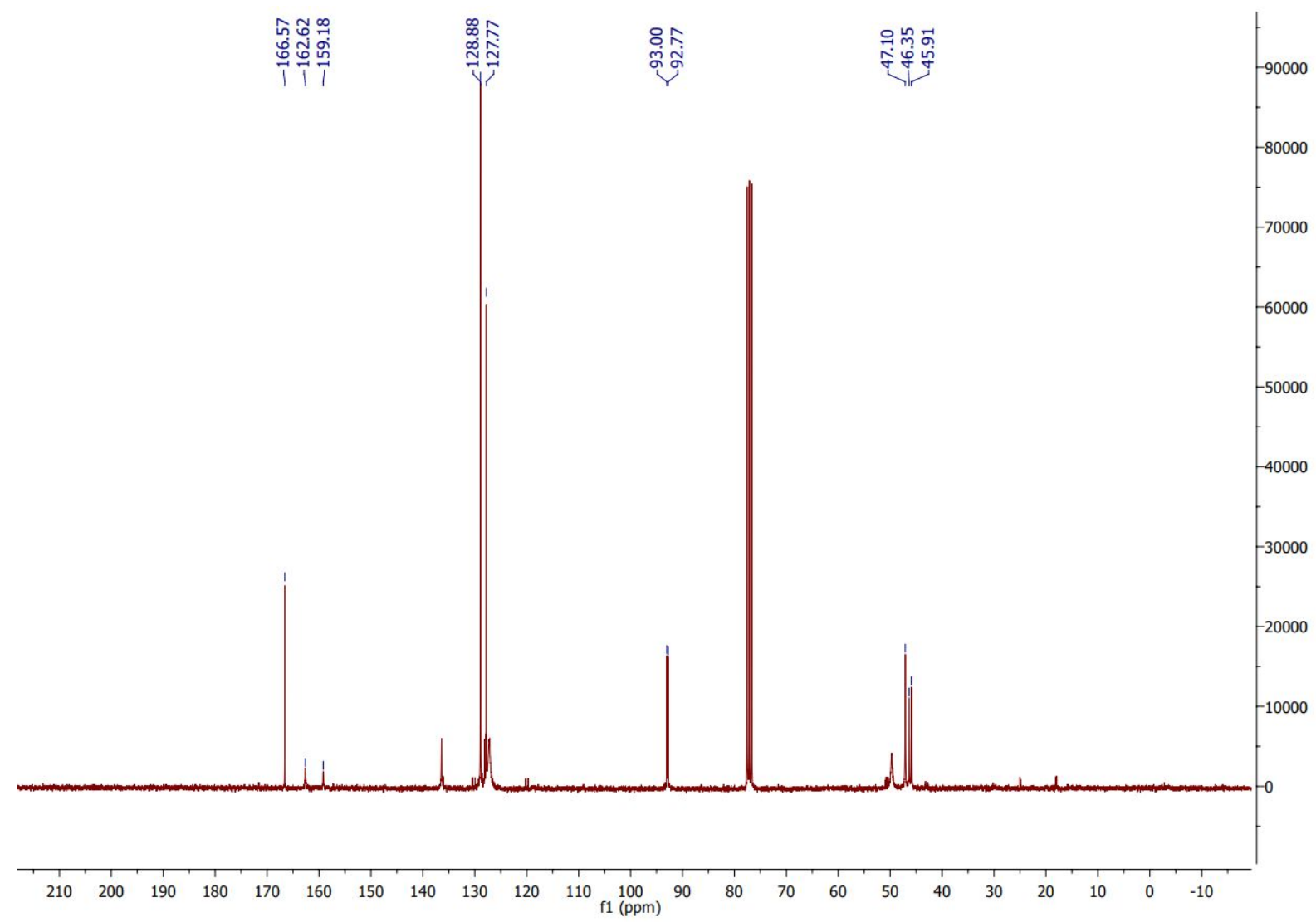

Figure S30. ${ }^{19} \mathrm{~F}$ NMR spectrum of $\mathbf{5 b}\left(282 \mathrm{MHz}, \mathrm{CDCl}_{3}\right)$.

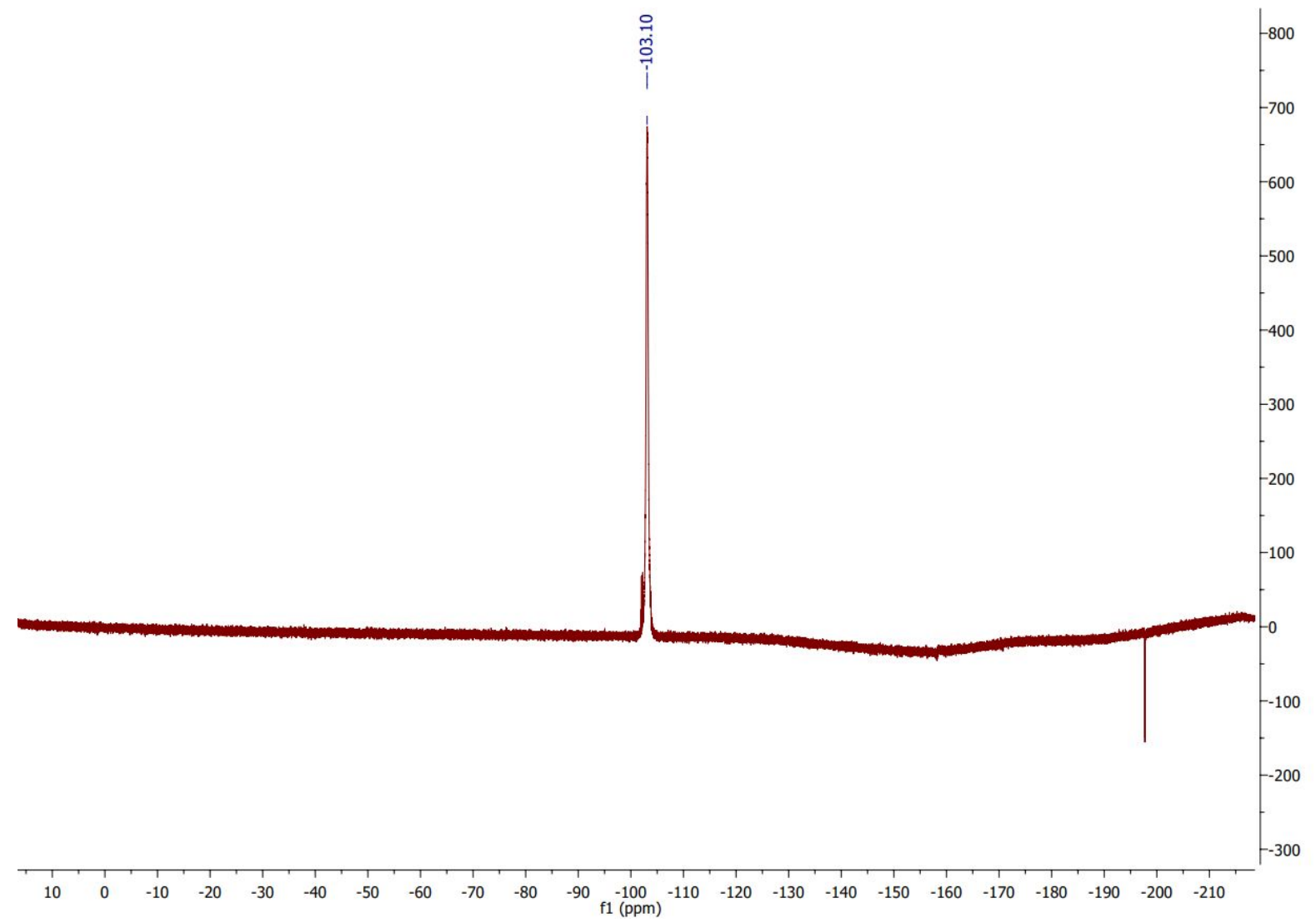


<smiles>C=C(F)CN(Cc1ccc([N+](=O)[O-])cc1)C(=O)C#N</smiles>

(5c) $N$-(2-fluoroallyl)-N-(4-nitrobenzyl)diazoacetamide

Figure S31. ${ }^{1} \mathrm{H}$ NMR spectrum of $5 \mathbf{c}\left(300 \mathrm{MHz}, \mathrm{CDCl}_{3}\right)$.

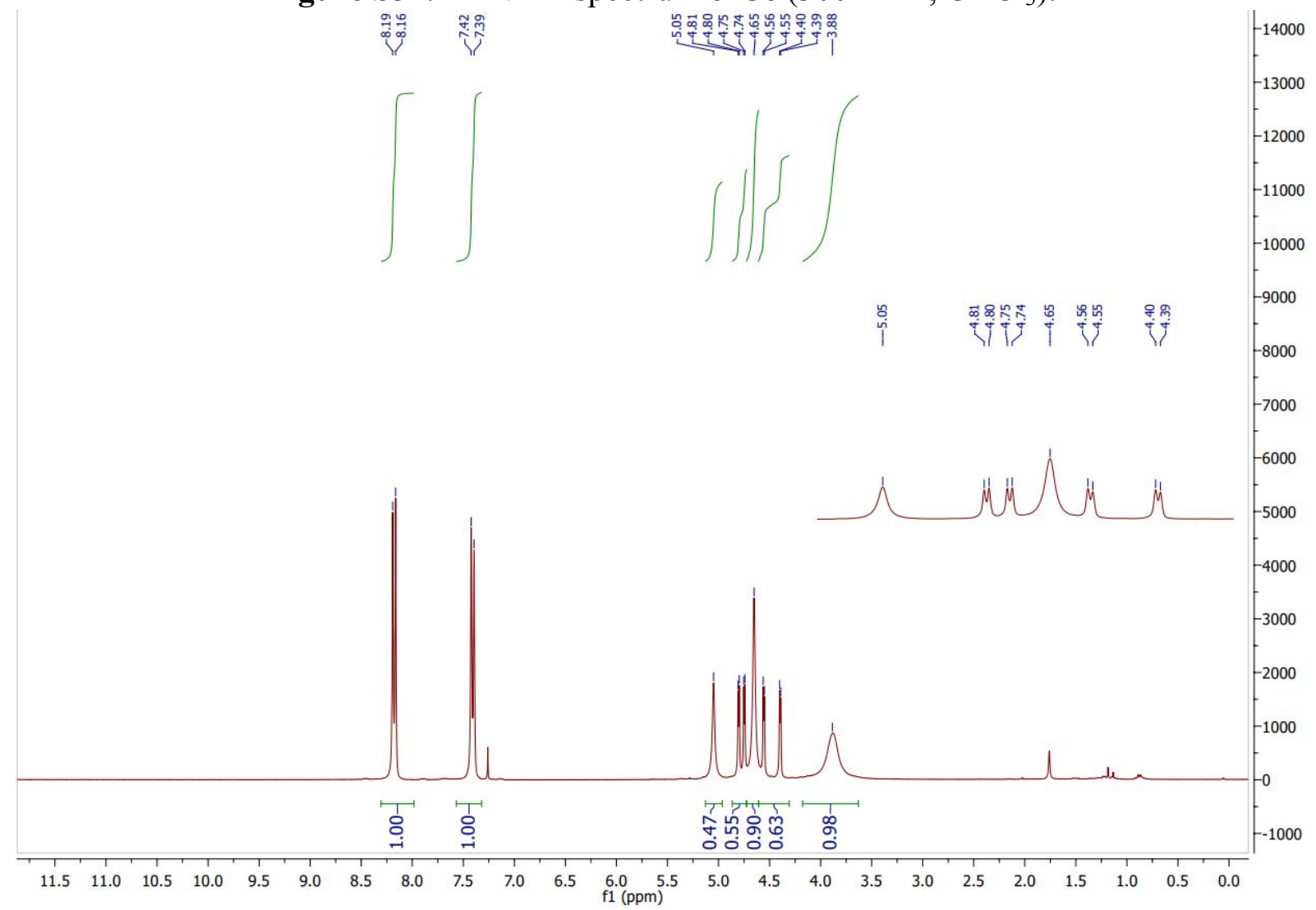


Figure S32. ${ }^{13} \mathrm{C} \mathrm{NMR}\{1 \mathrm{H}\}$ spectrum of $\mathbf{5 c}\left(75 \mathrm{MHz}, \mathrm{CDCl}_{3}\right)$.

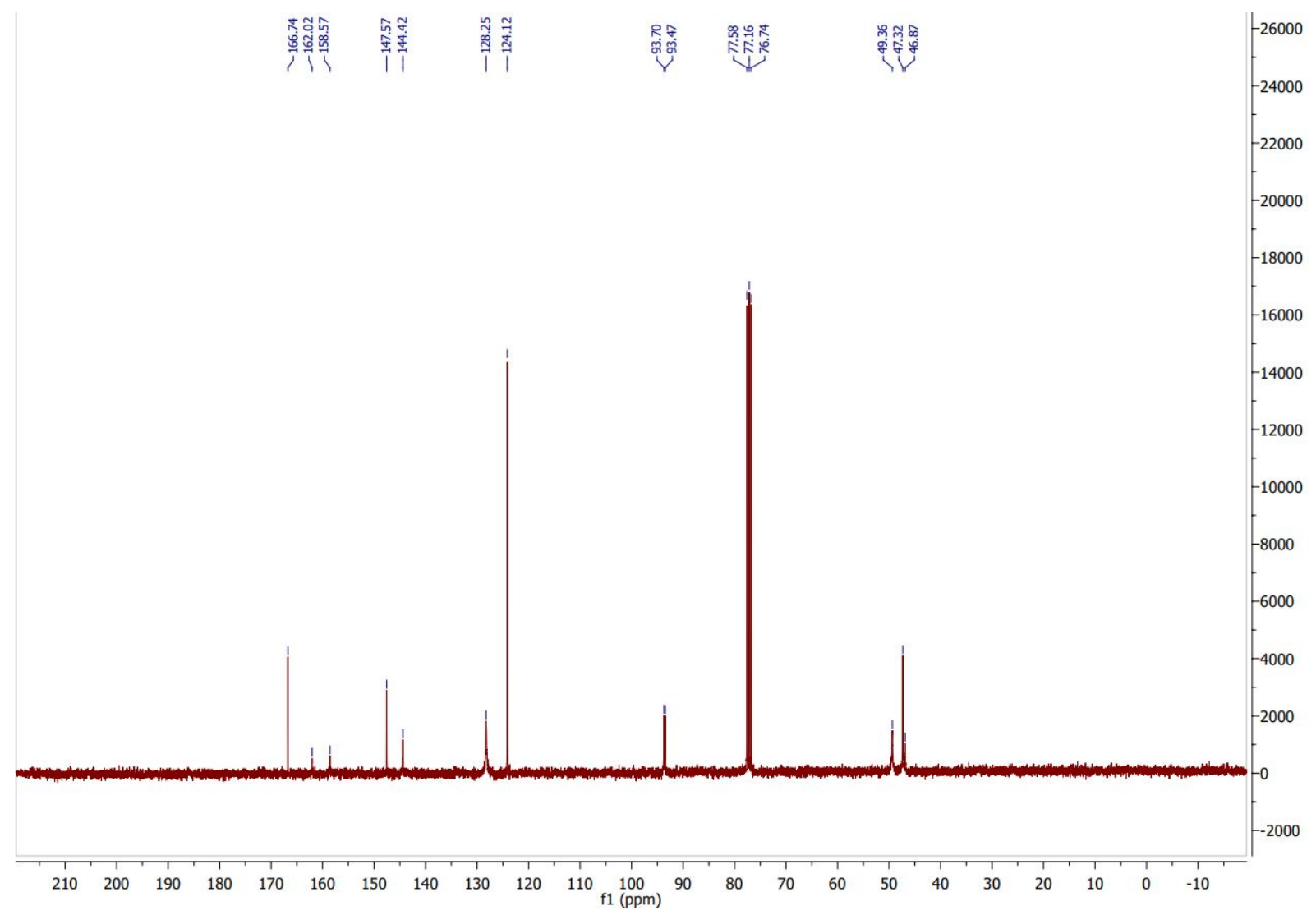

Figure S33. ${ }^{19} \mathrm{~F}$ NMR spectrum of $\mathbf{5 c}\left(282 \mathrm{MHz}, \mathrm{CDCl}_{3}\right)$.

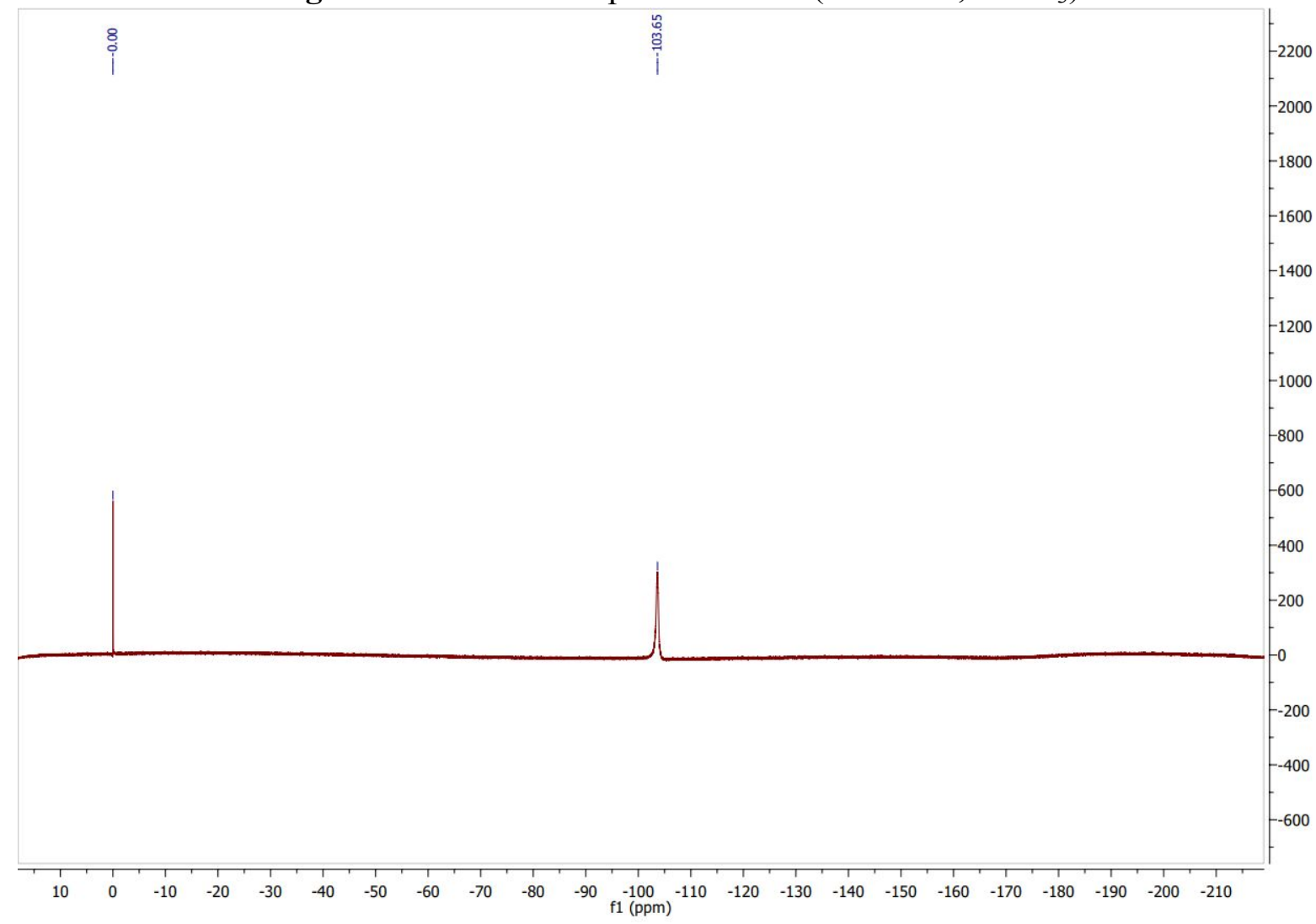


<smiles>C=C(F)CN(Cc1ccc(Cl)cc1)C(=O)C=N</smiles>

(5d) N-(2-fluoroallyl)-N-(4-chlorobenzyl)diazoacetamide

Figure S34. ${ }^{1} \mathrm{H}$ NMR spectrum of $\mathbf{5 d}\left(300 \mathrm{MHz}, \mathrm{CDCl}_{3}\right)$.

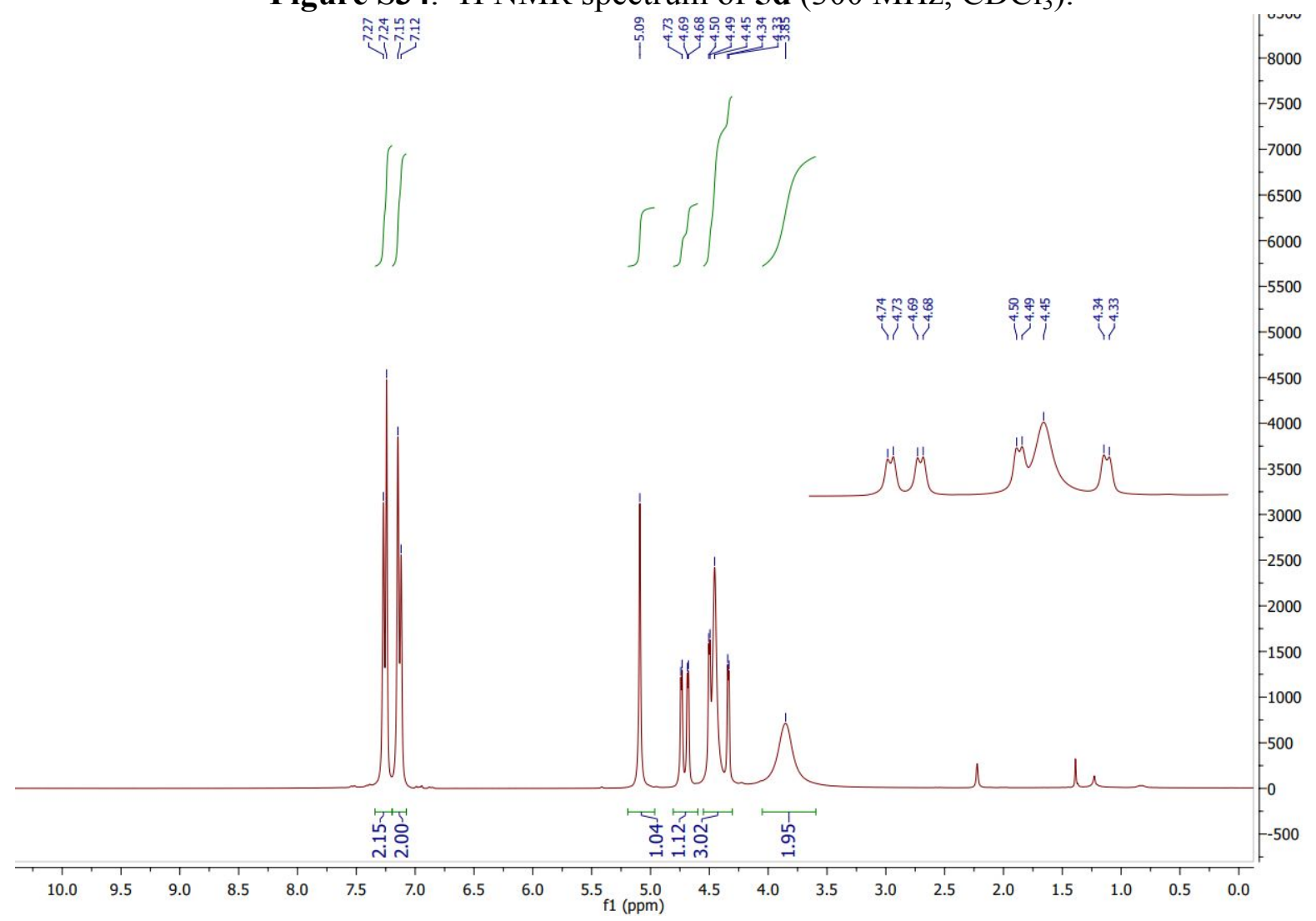


Figure S35. ${ }^{13} \mathrm{C} \mathrm{NMR}\{1 \mathrm{H}\}$ spectrum of $\mathbf{5 d}\left(75 \mathrm{MHz}, \mathrm{CDCl}_{3}\right)$.

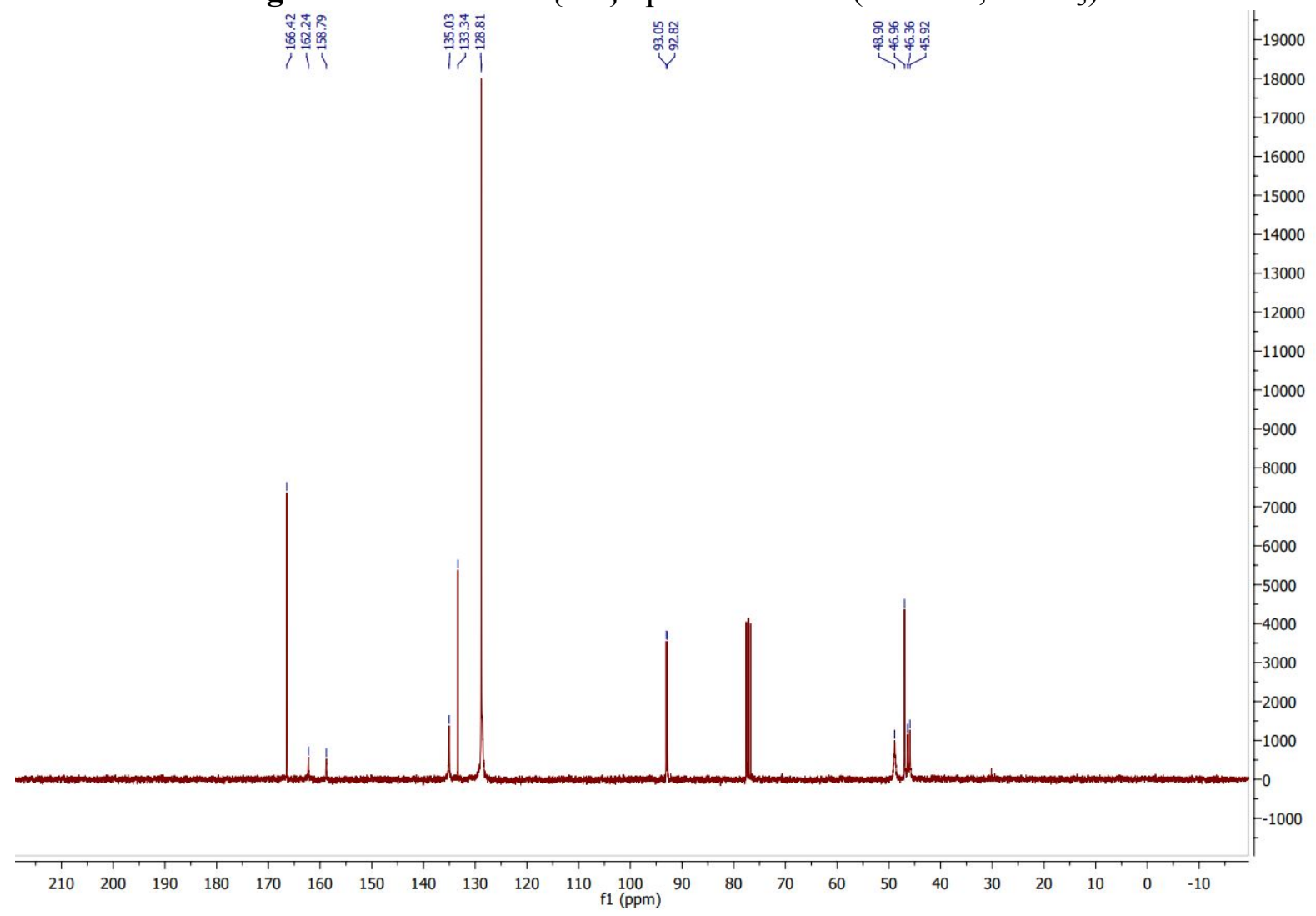

Figure S36. ${ }^{19} \mathrm{~F}$ NMR spectrum of $\mathbf{5 d}\left(282 \mathrm{MHz}, \mathrm{CDCl}_{3}\right)$.

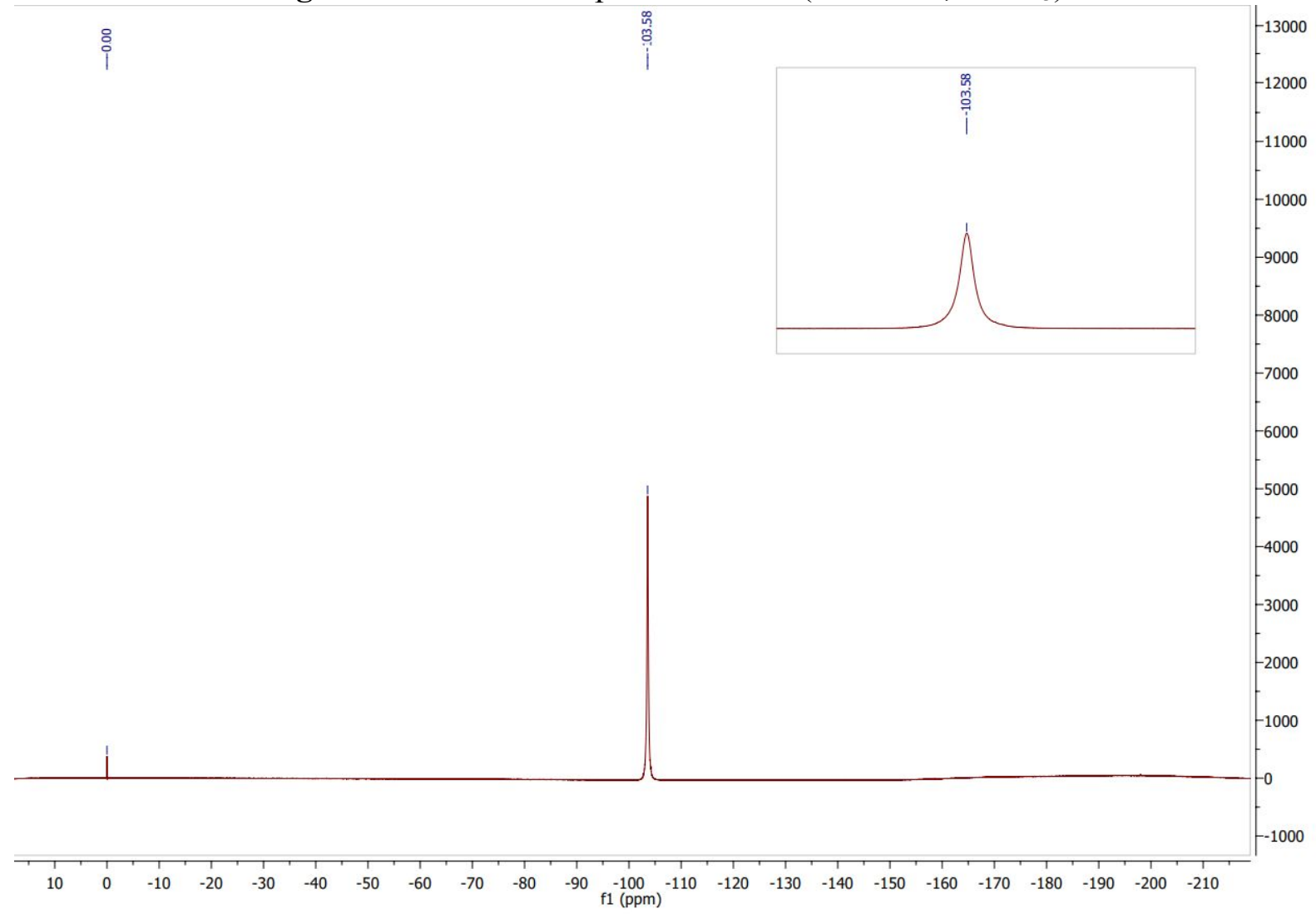


<smiles>O=C1C[C@H](F)CN1Cc1ccccc1</smiles>

((-)-6b) 3-benzyl-5-fluoro-3-azabicyclo[3.1.0] hexan-2-one

Figure S37. ${ }^{1} \mathrm{H}$ NMR spectrum of (-)-6b (300 MHz, $\left.\mathrm{CDCl}_{3}\right)$.

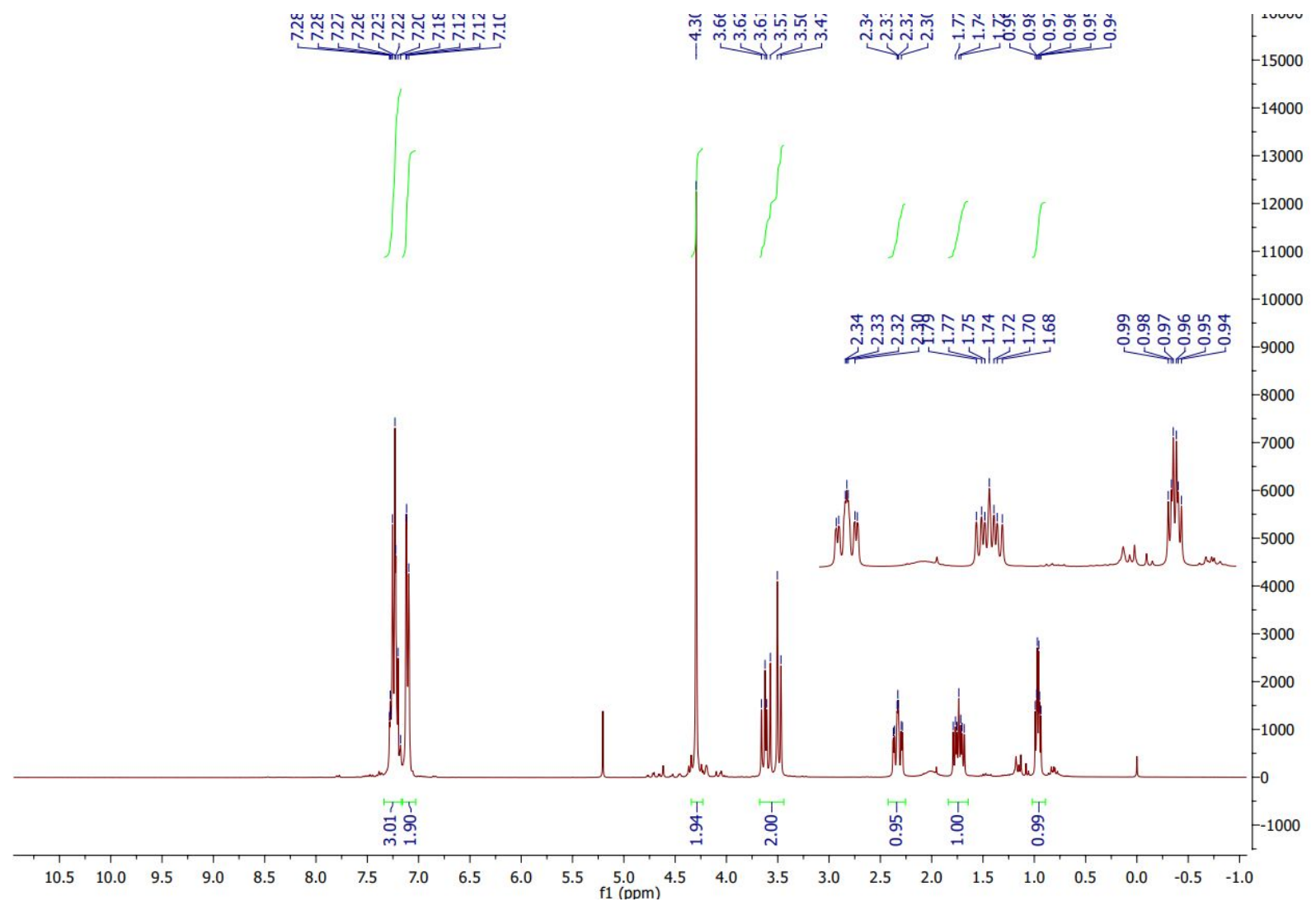


Figure S38. ${ }^{13} \mathrm{C}$ NMR $\{1 \mathrm{H}\}$ spectrum of (-)-6b (75 MHz, $\left.\mathrm{CDCl}_{3}\right)$.

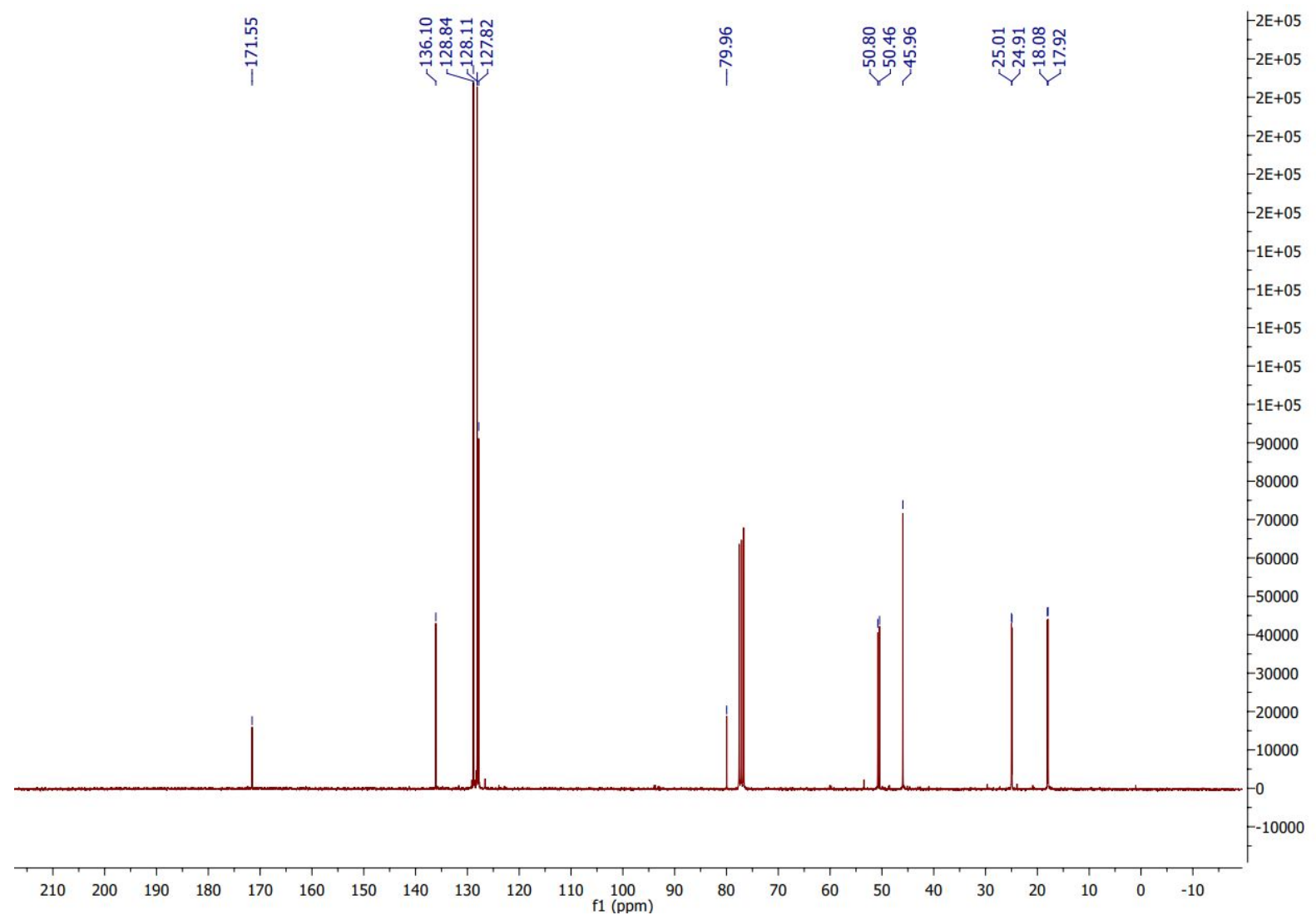


Figure S39. ${ }^{19} \mathrm{~F}$ NMR spectrum of (-)-6b $\left(282 \mathrm{MHz}, \mathrm{CDCl}_{3}\right)$.

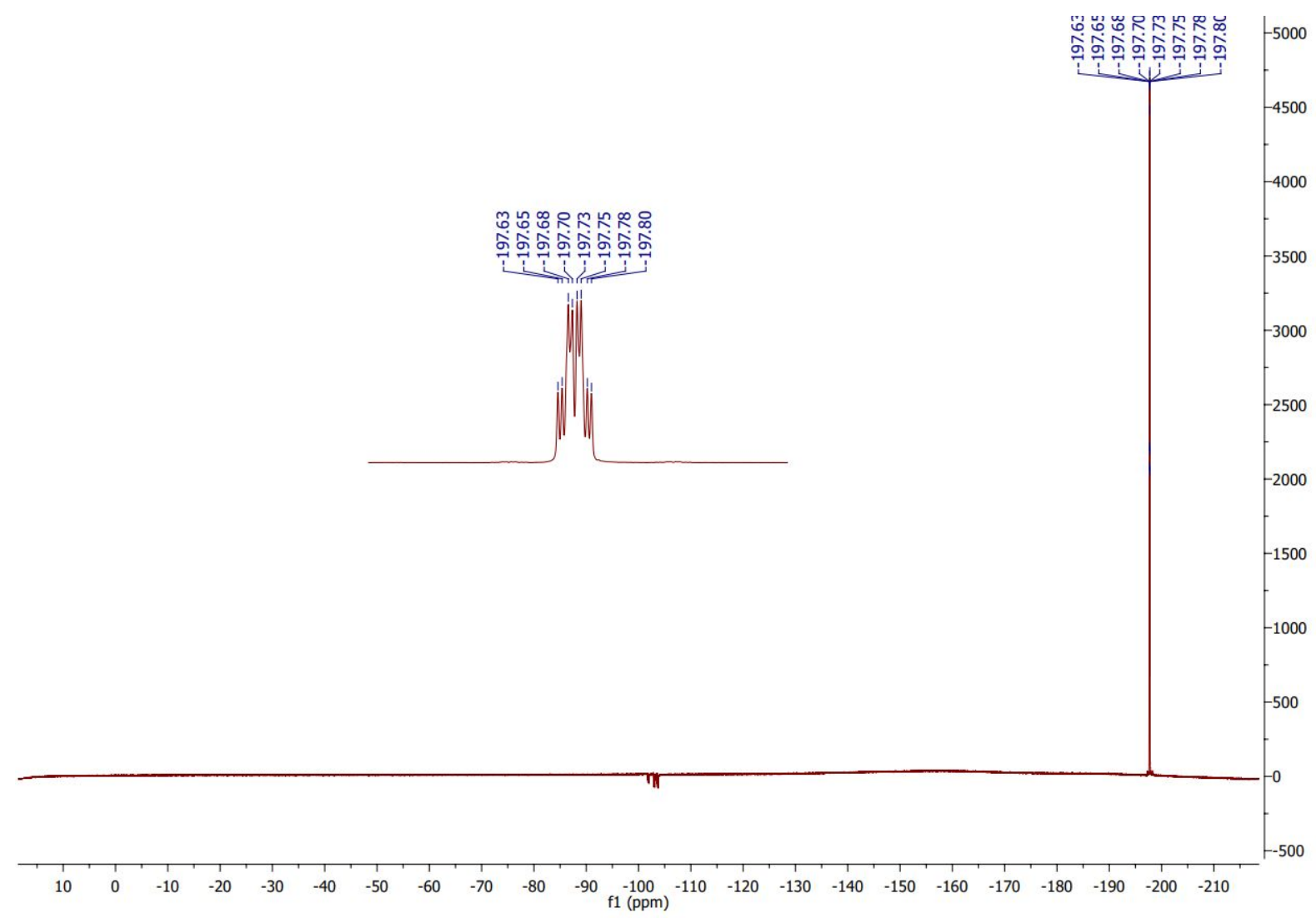

Figure S40: Analytical SFC after preparative chiral SFC separation. The second enantiomer ((-)-6b, $t_{R}$ $=1.12 \mathrm{~min}$ ) was used to continue the synthesis.

\section{Report SFC-MS}

NotebookID: CS048B_ISO1

Remark: Check EE purity, (99.68\% isomer 1$),(0.32 \%$ isomer 2$)$.

Method description:

System: UPC ${ }^{2}$

Column: Chiralpak AS-3 3um 100x4.6mm

Flow: $3.5 \mathrm{ml} / \mathrm{min}$

Mobile phase: $90 \% \mathrm{CO}_{2} / 10 \%$ iPrOH hold $3 \mathrm{~min}$.

Temperature: $35^{\circ} \mathrm{C}$

Pressure outlet: 103b

Conclusion/Chromatogram/spectra

$\begin{array}{lll}\text { Rt }(0.83 \mathrm{~min}) & \text { UV: } 99.68 \% & \text { isomer } 1 \\ \text { Rt }(1.20 \mathrm{~min}) & \text { UV: } 0.32 \% & \text { isomer } 2\end{array}$




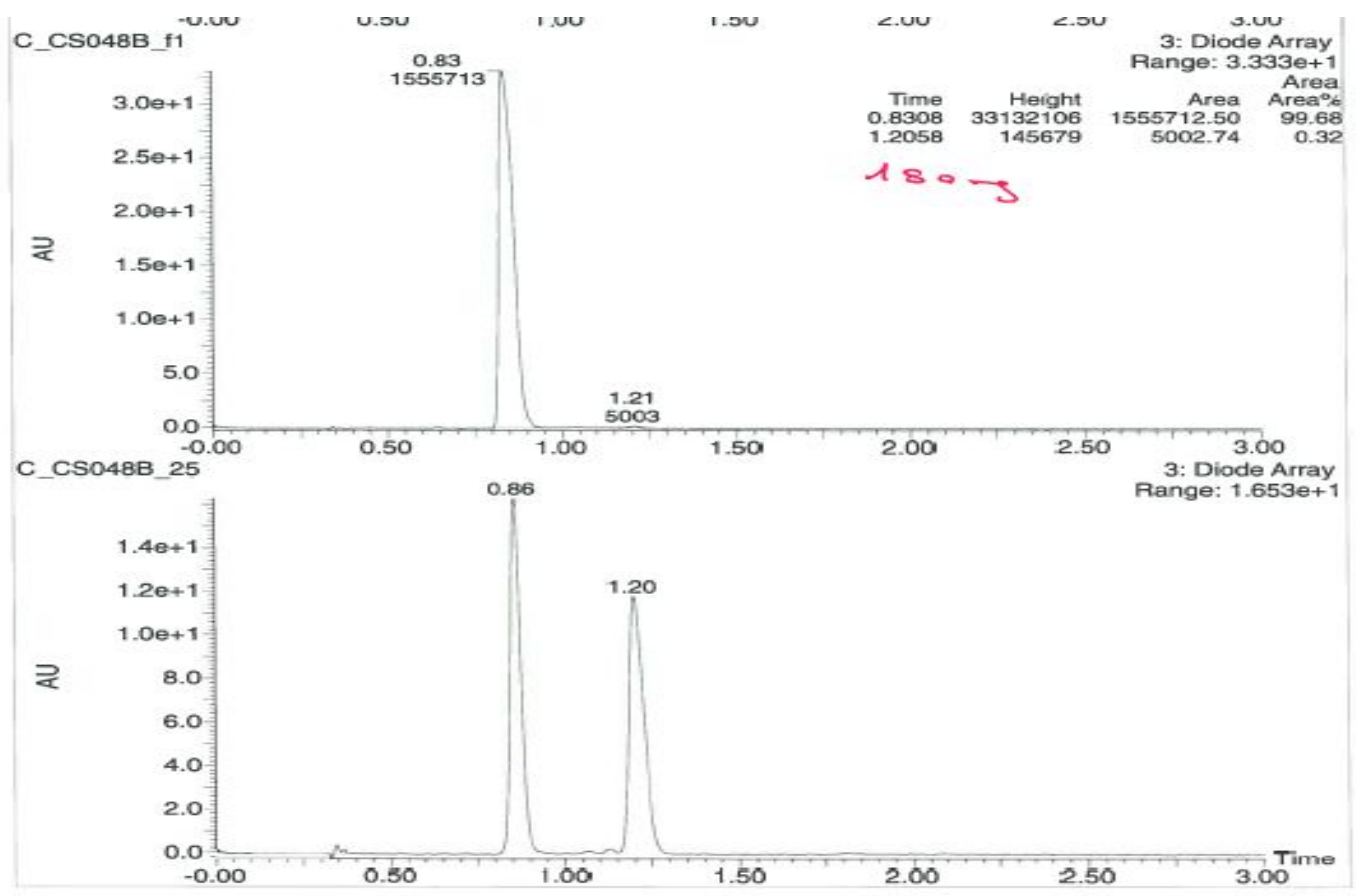

\section{Report SFC-MS}

NotebookID: CSO48B_ISO2

Remark: Check EE purity, (0.30\% isomer 1$),(99.7 \%$ isomer 2$)$.

Method description:

System: UPC ${ }^{2}$

Column: Chiralpak AS-3 3um 100x4.6mm

Flow: $3.5 \mathrm{ml} / \mathrm{min}$

Mobile phase: $90 \% \mathrm{CO}_{2} / 10 \%$ iPrOH hold $3 \mathrm{~min}$.

Temperature: $35^{\circ} \mathrm{C}$

Pressure outlet: 103b

Conclusion/Chromatogram/spectra

$\begin{array}{lll}\text { Rt }(1.12 \mathrm{~min}) & \text { UV: } 99.70 \% & \text { isomer } 2 \\ \text { Rt }(0.86 \mathrm{~min}) & \text { UV: } 0.30 \% & \text { isomer } 1\end{array}$


AS3 $100 \times 4.6 \mathrm{~mm}$, iso $10 \%$ iPrOH_NEAT, $3.5 \mathrm{mi} / \mathrm{min}$ C_CSO48B $\mathrm{f} 2$

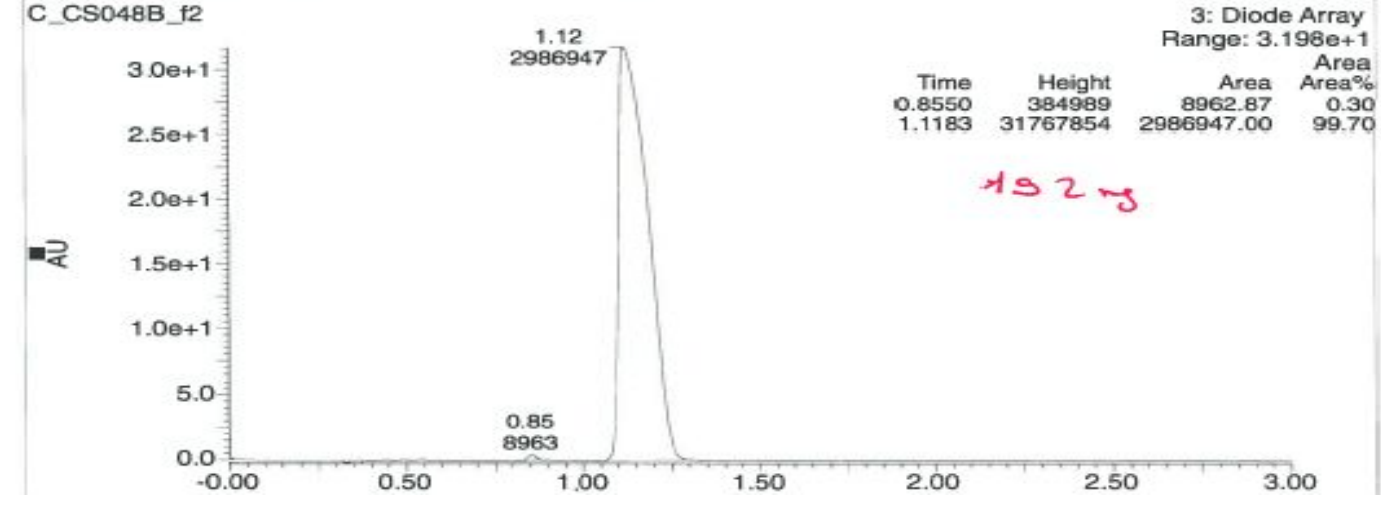

24-Mar-201711:14:00 3: Diode Array Area $\begin{array}{rrrr}\text { Time } & \text { Height } & \text { Area } & \text { Area\% } \\ 0.8550 & 384989 & 8962.87 & 0.30\end{array}$

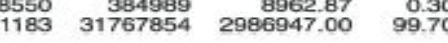
$192 \mathrm{ng}$

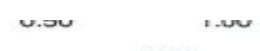

s.ou

c.ur

$1.4 e+1$
$1.2 e+1$
$1.0 e+1$
8.0
6.0
4.0
2.0
0.0
-0.00 
Enantioselective Cyclopropanation:

Figure S41. HPLC analysis of $\mathbf{6 b}$ (racemic).

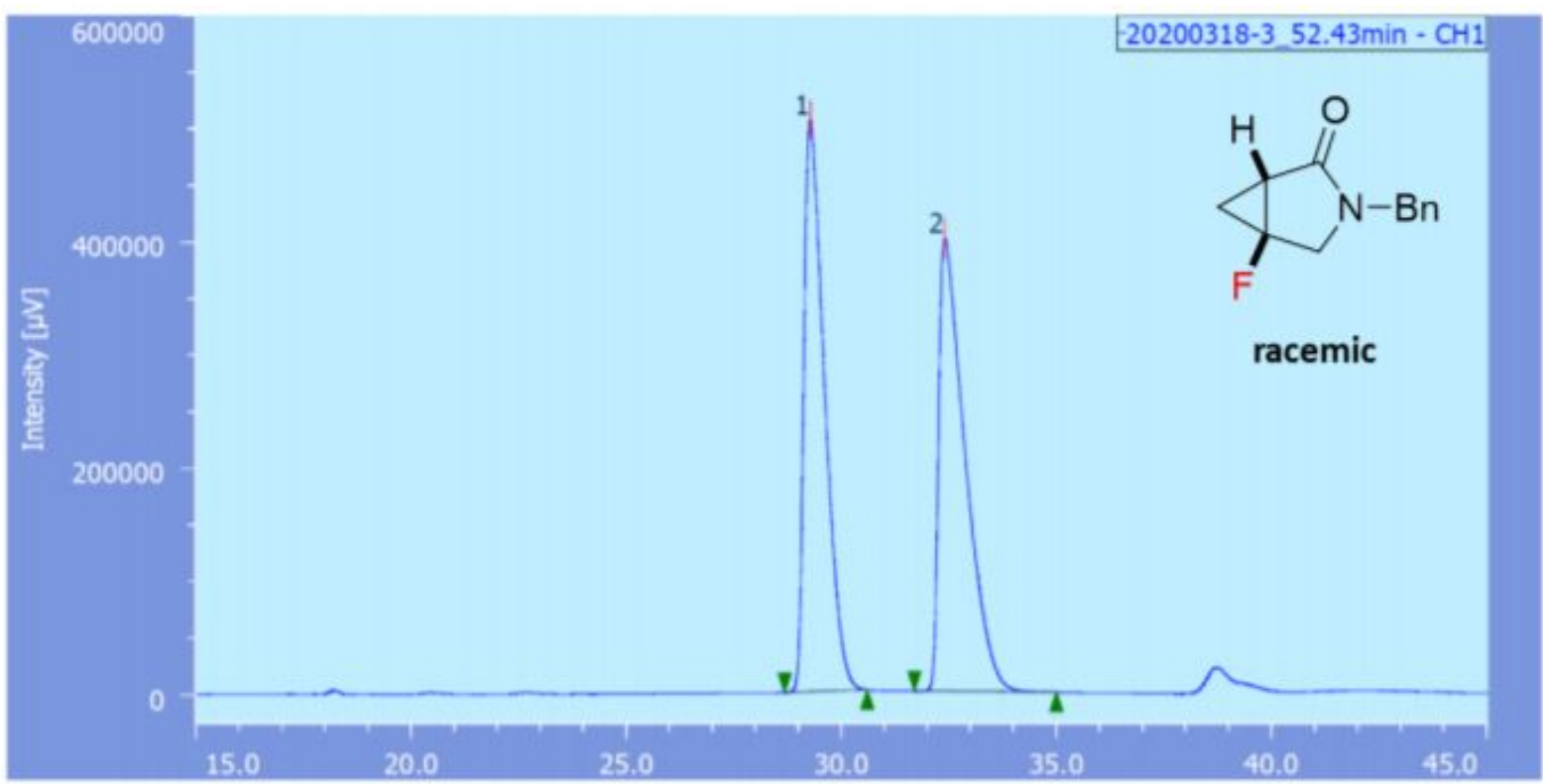

\begin{tabular}{|c|r|r|r|r|r|}
\hline PEAK & RT $[\mathrm{min}]$ & AREA $[\mu \mathrm{V} \cdot \mathrm{sec}]$ & HEIGHT $[\mu \mathrm{V}]$ & AREA\% & HEIGHT\% \\
\hline 1 & 29.275 & 16726608 & 505007 & 49.468 & 55.783 \\
\hline 2 & 32.408 & 17086152 & 400295 & 50.532 & 44.217 \\
\hline
\end{tabular}


Figure S42. HPLC analysis of $\mathbf{6 b}$ (chiral). ee $=77 \%$

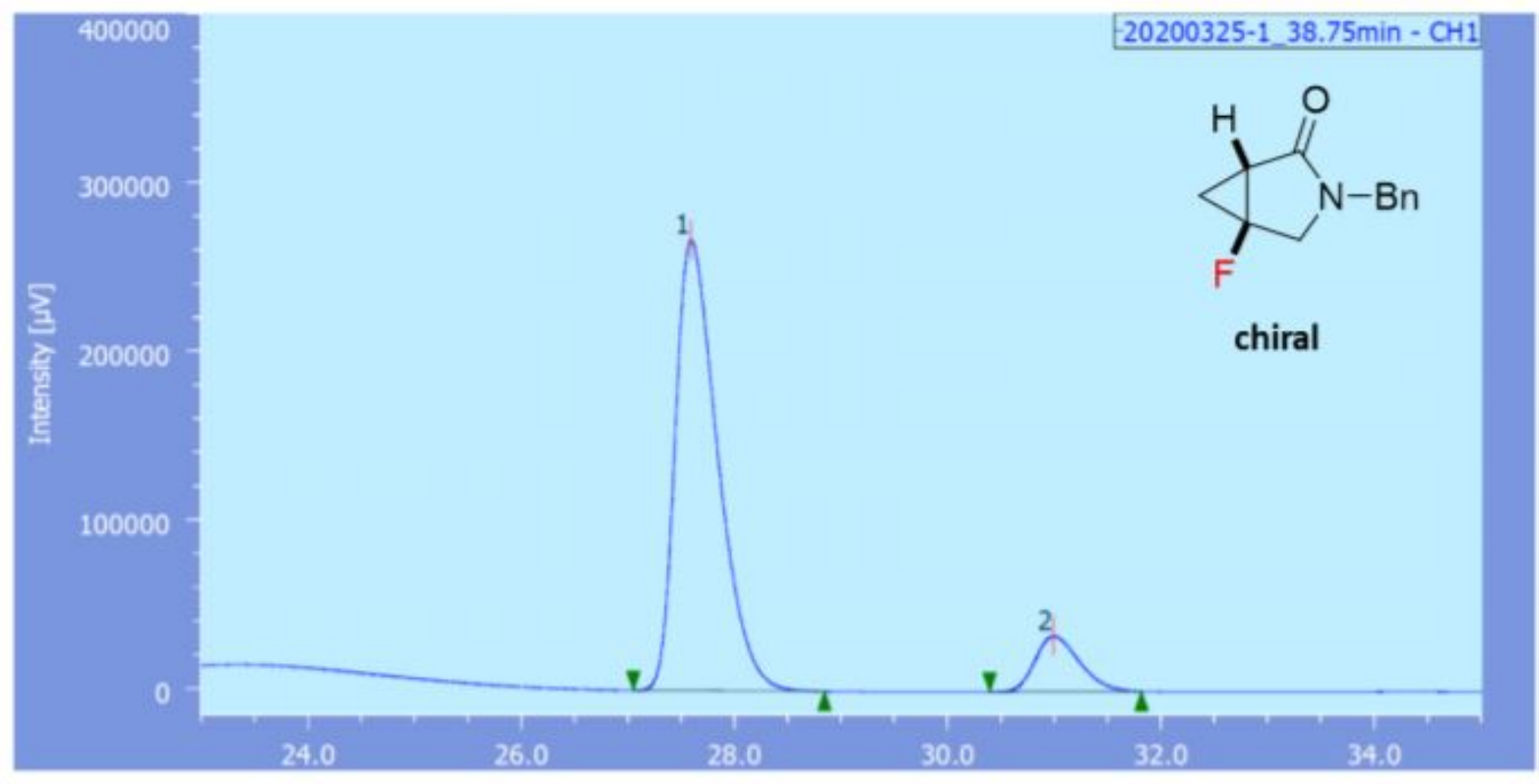

\begin{tabular}{|c|r|r|r|r|r|}
\hline PEAK & RT $[\mathrm{min}]$ & AREA $[\mu \mathrm{V}$-sec] & HEIGHT $[\mu \mathrm{V}]$ & AREA\% & HEIGHT\% \\
\hline 1 & 27.592 & 7388862 & 267208 & 88.491 & 89.015 \\
\hline 2 & 30.992 & 960953 & 32974 & 11.509 & 10.985 \\
\hline
\end{tabular}


<smiles>N#CC1CC(F)(F)CN1Cc1ccccc1</smiles>

((-)-cis-7b) 3-benzyl-5-fluoro-3-azabicyclo[3.1.0]hexane-2-carbonitrile

Figure S43. ${ }^{1} \mathrm{H}$ NMR spectrum of (-)-7b $\left(300 \mathrm{MHz}, \mathrm{CDCl}_{3}\right)$.

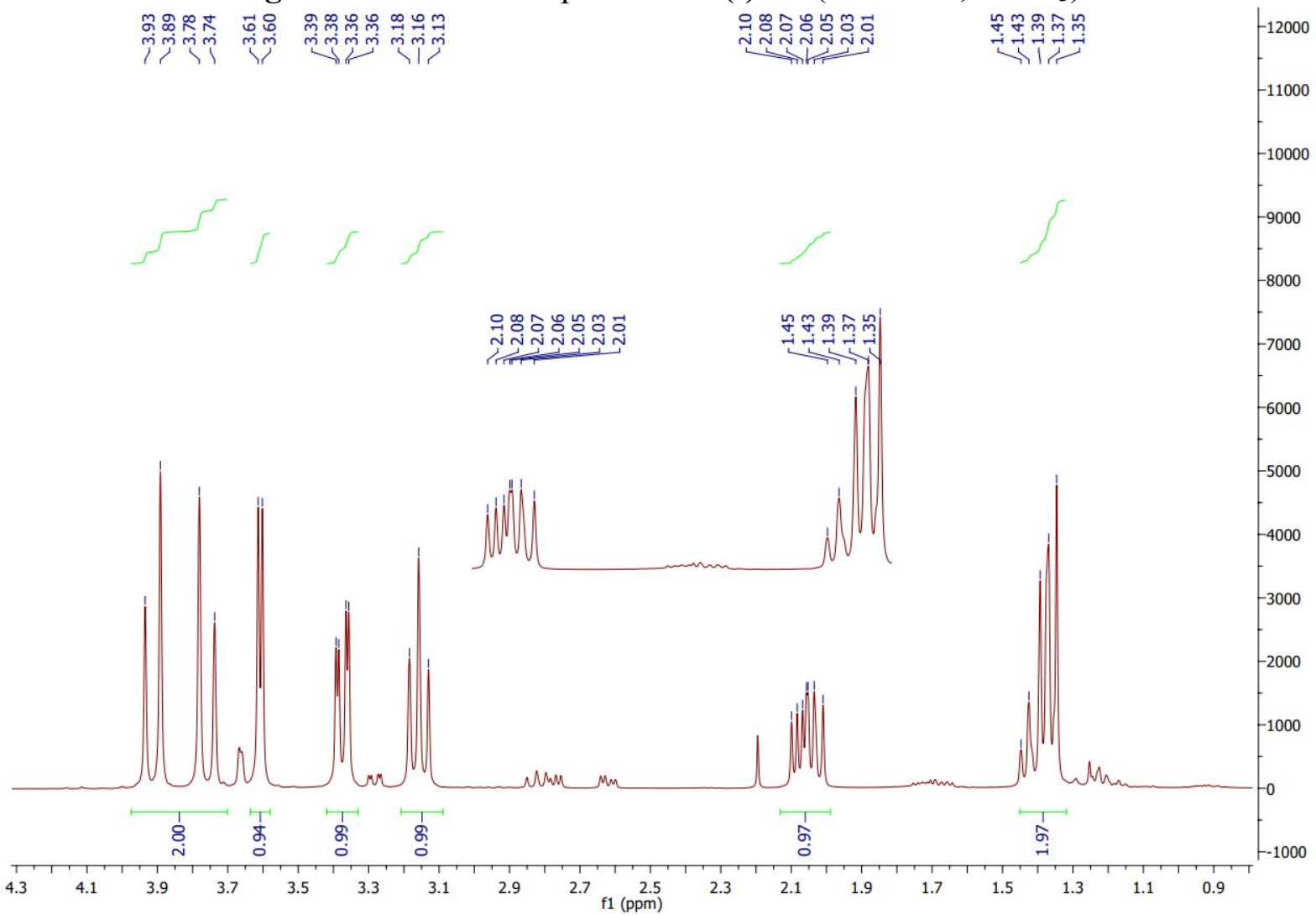


Figure S44. ${ }^{13} \mathrm{C} \mathrm{NMR}\{1 \mathrm{H}\}$ spectrum of (-)-7b (75 $\left.\mathrm{MHz}, \mathrm{CDCl}_{3}\right)$.

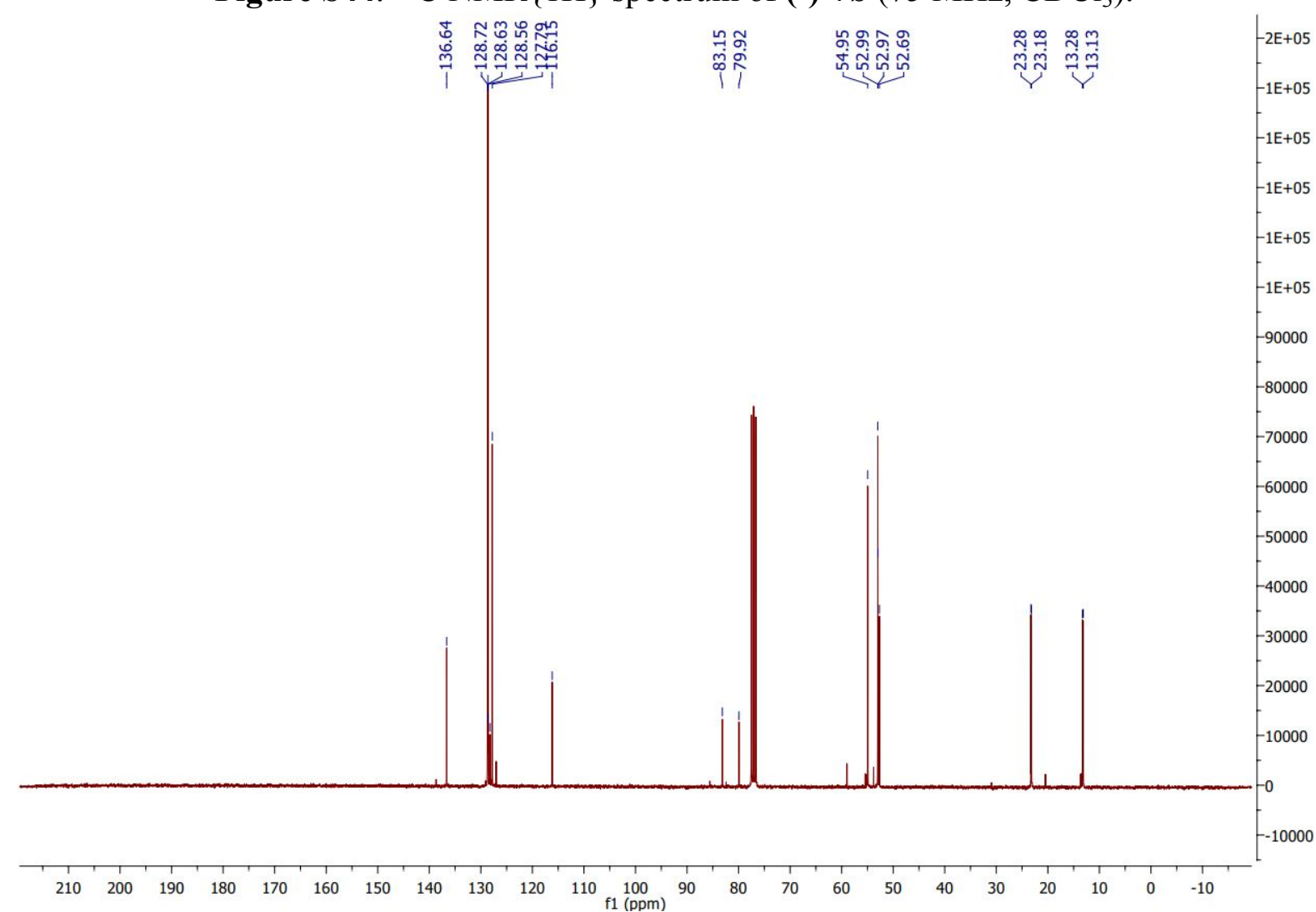

Figure S45. ${ }^{19} \mathrm{~F}$ NMR spectrum of (-)-7b (282 $\left.\mathrm{MHz}, \mathrm{CDCl}_{3}\right)$.

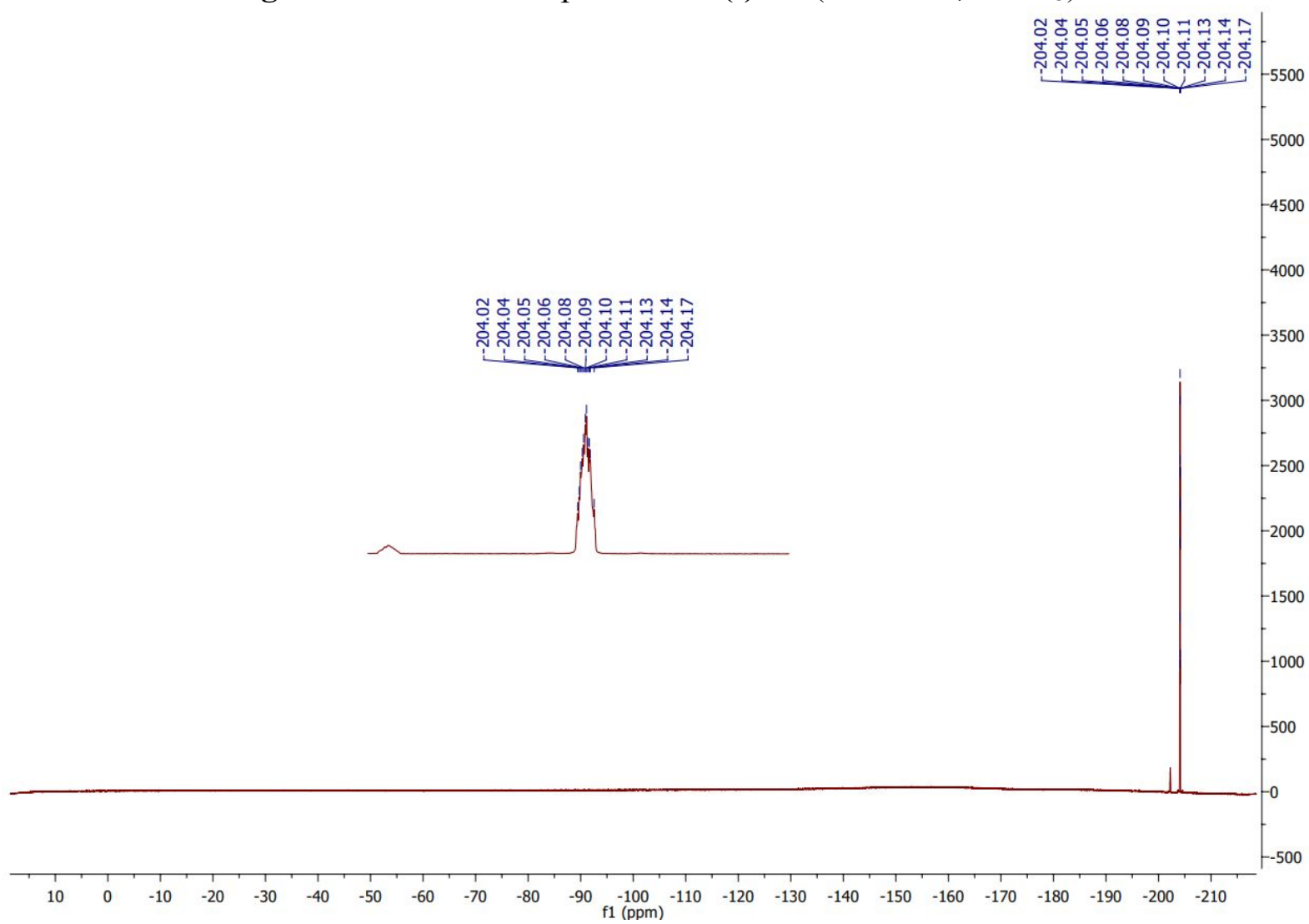


<smiles>O=C(O)C1CC(F)(F)CN1</smiles>

((-)-cis-8b) cis-5-fluoro-3-azabicyclo[3.1.0]hexane-2-carboxylic acid

Figure S46. ${ }^{1} \mathrm{H}$ NMR spectrum of (-)-8b (300 MHz, $\left.\mathrm{D}_{2} \mathrm{O}\right)$.

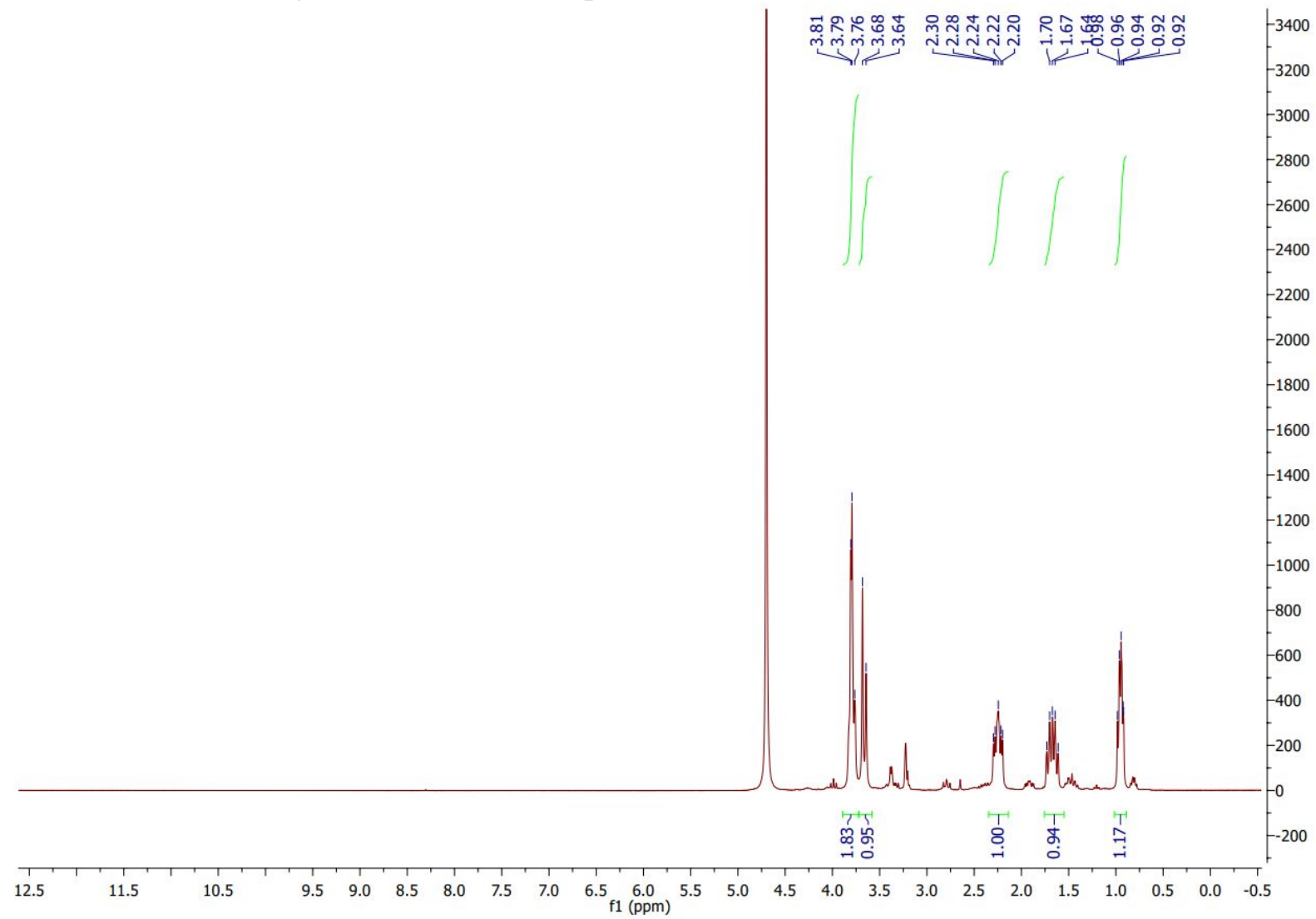


Figure S47. ${ }^{13} \mathrm{C}\{1 \mathrm{H}\}$ NMR spectrum of (-)-8b (75 MHz, $\left.\mathrm{D}_{2} \mathrm{O}\right)$.

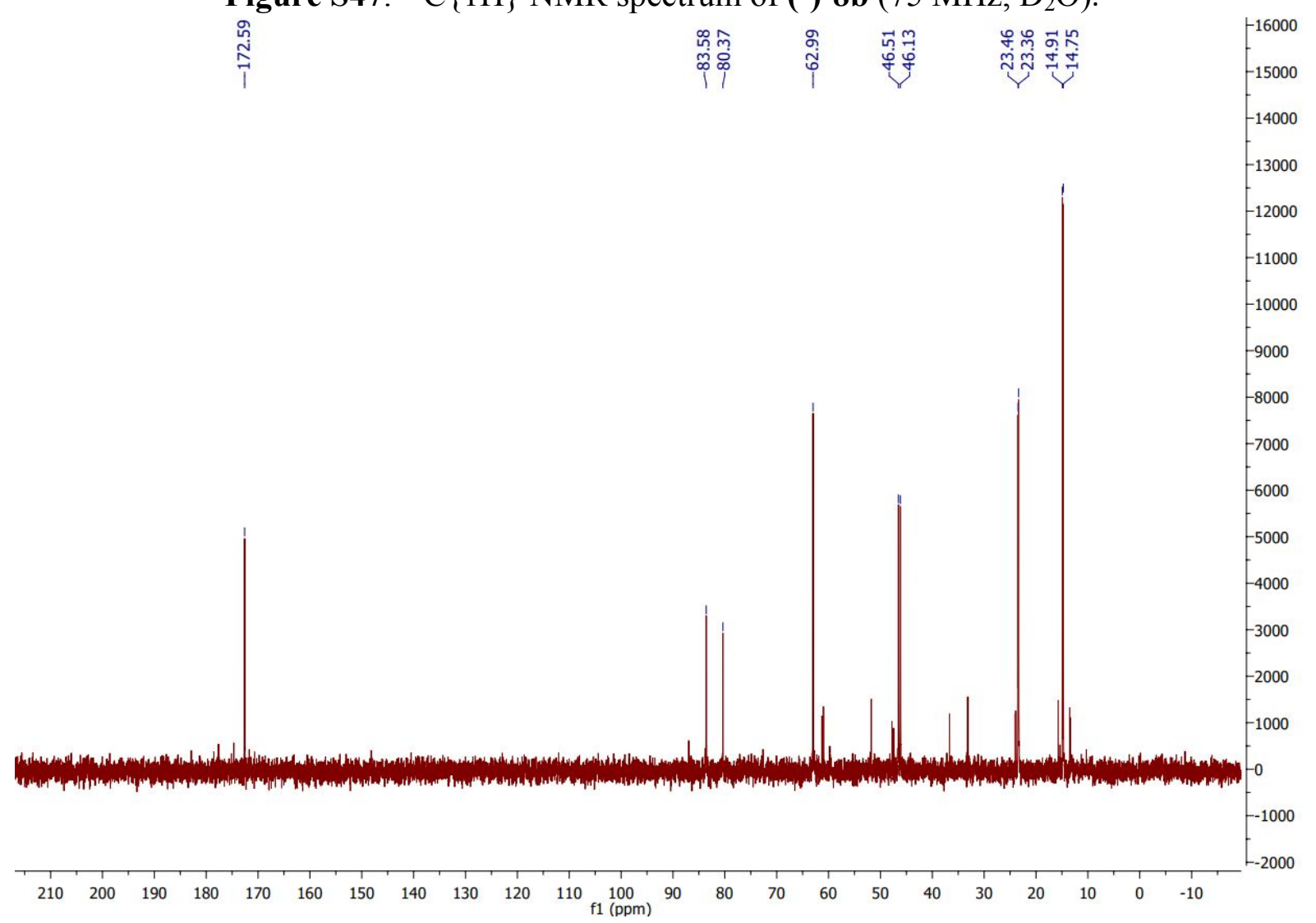

Figure S48. ${ }^{19} \mathrm{~F}$ NMR spectrum of (-)-8b (282 MHz, $\left.\mathrm{D}_{2} \mathrm{O}\right)$.

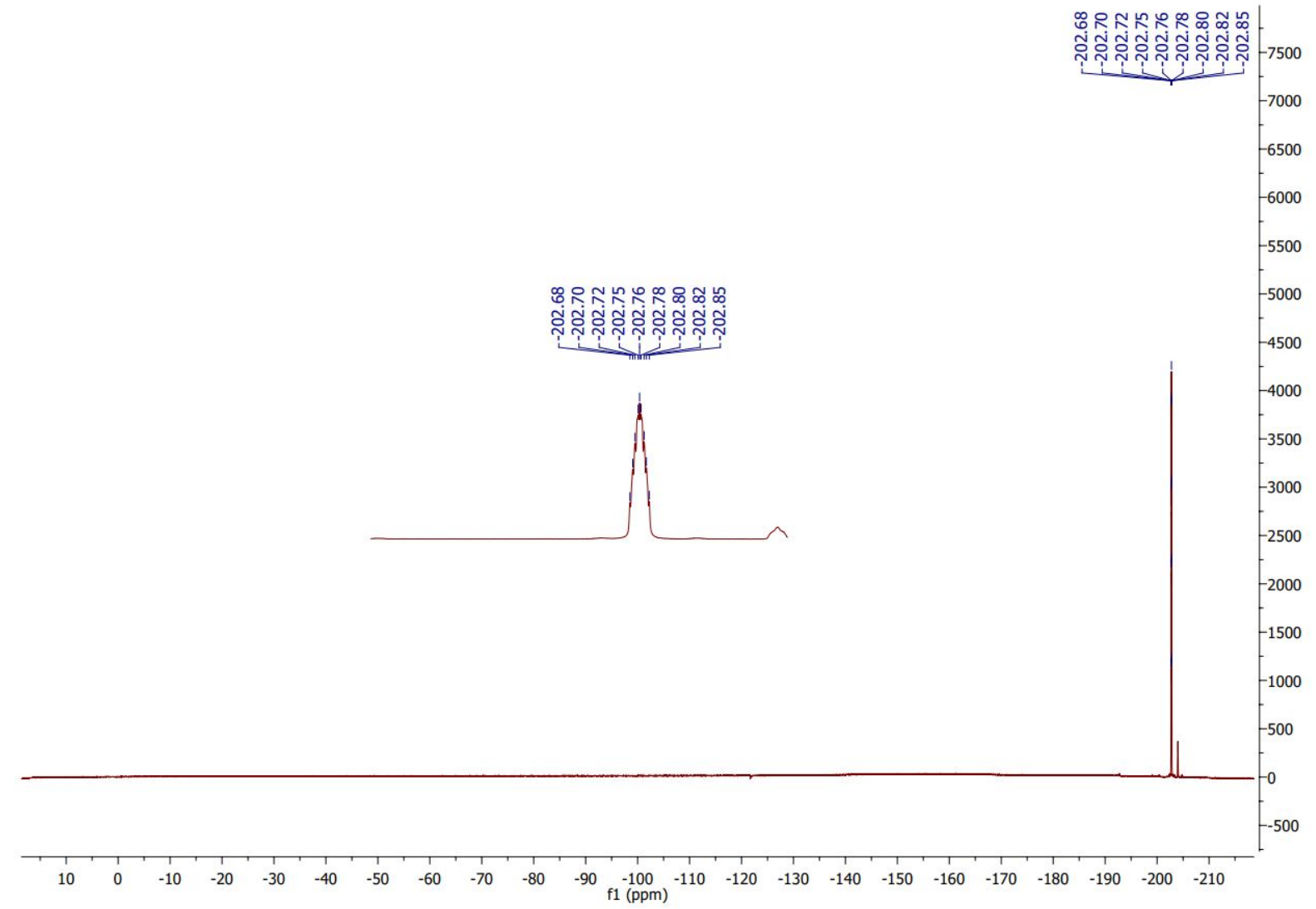


<smiles>CC(C)(C)OC(=O)N1CC(F)(F)[C@H]2CCCC21</smiles>

((-)-cis-9b) cis-3-(tert-butoxycarbonyl)-5-fluoro-3-azabicyclo[3.1.0] hexane-2-carboxylic acid

Figure S49. ${ }^{1} \mathrm{H}$ NMR spectrum of (-)-9b (300 MHz, $\left.\mathrm{CDCl}_{3}\right)$.

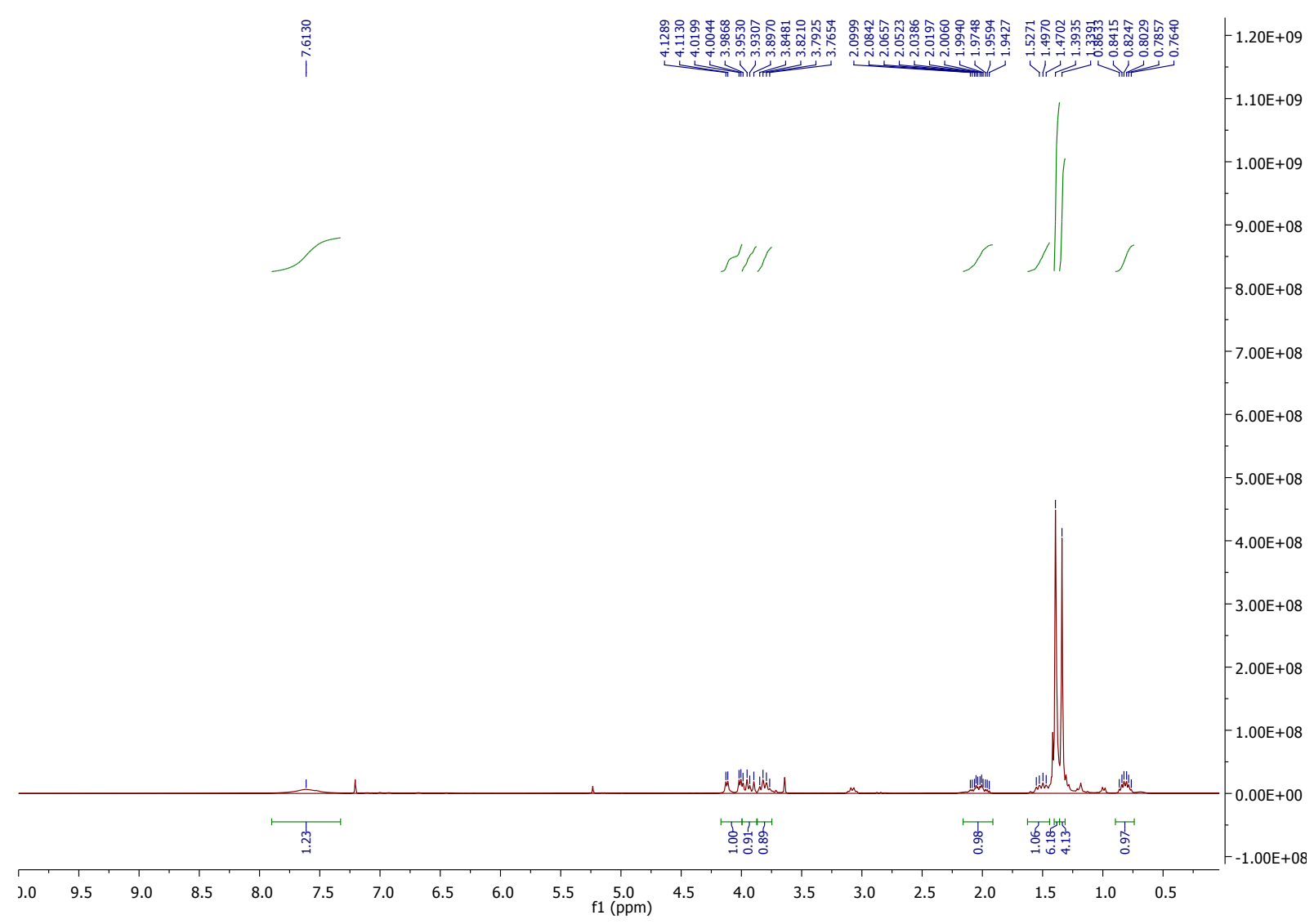


Figure S50. ${ }^{13} \mathrm{C}$ NMR $\{1 \mathrm{H}\}$ spectrum of (-)-9b $\left(75 \mathrm{MHz}, \mathrm{CDCl}_{3}\right)$.

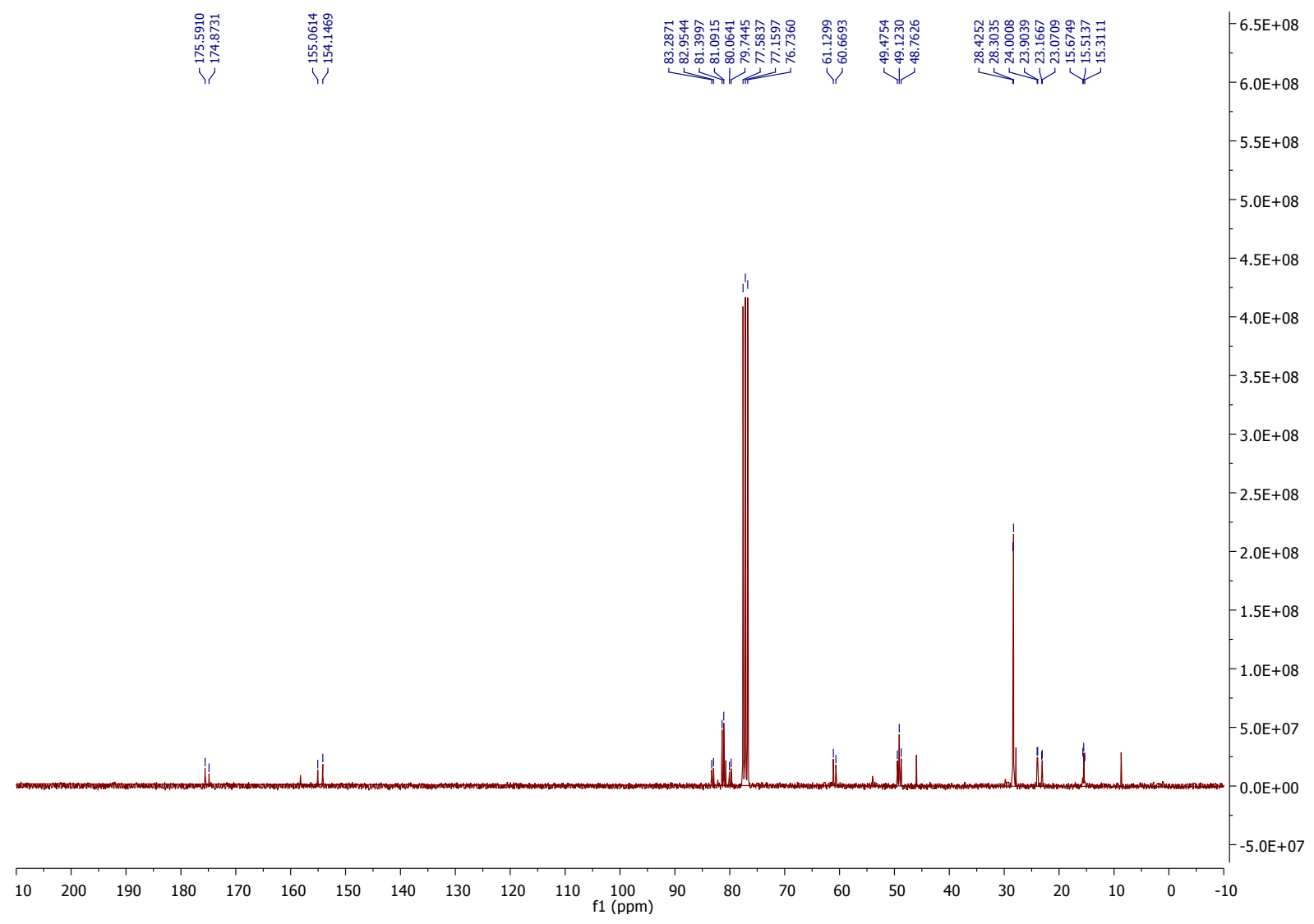

Figure S51. ${ }^{19}$ F NMR spectrum of (-)-9b (282 $\left.\mathrm{MHz}, \mathrm{CDCl}_{3}\right)$.

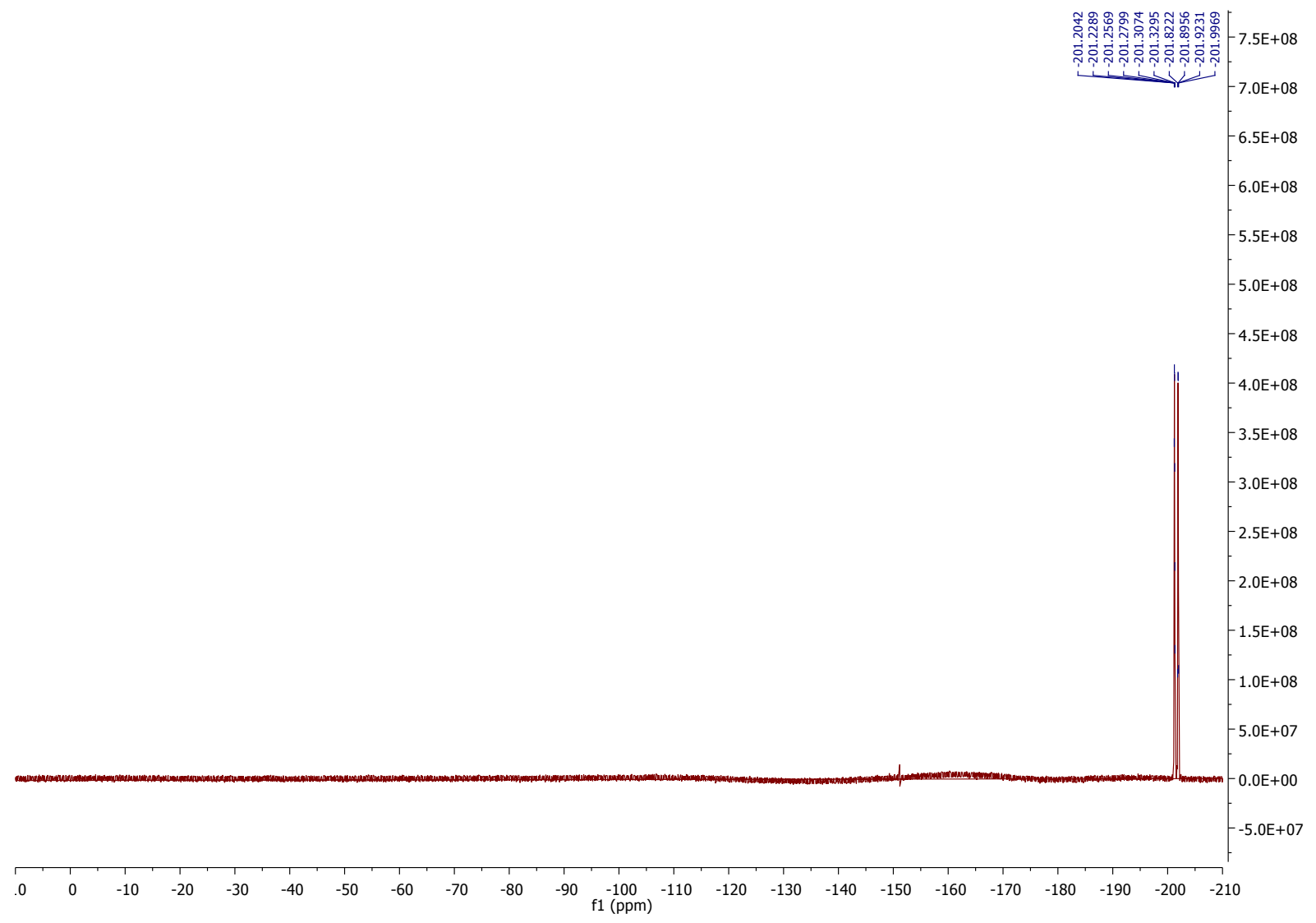




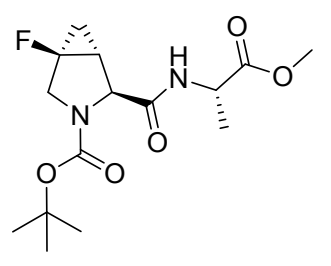

((-)-10) tert-butyl 1-fluoro-4-(((S)-1-methoxy-1-oxopropan-2-yl)carbamoyl)-3-azabicyclo[3.1.0]hexane-3-carboxylate

Figure S52. ${ }^{1} \mathrm{H}$ NMR spectrum of (-)-10 (300 MHz, $\left.\mathrm{CDCl}_{3}\right)$.

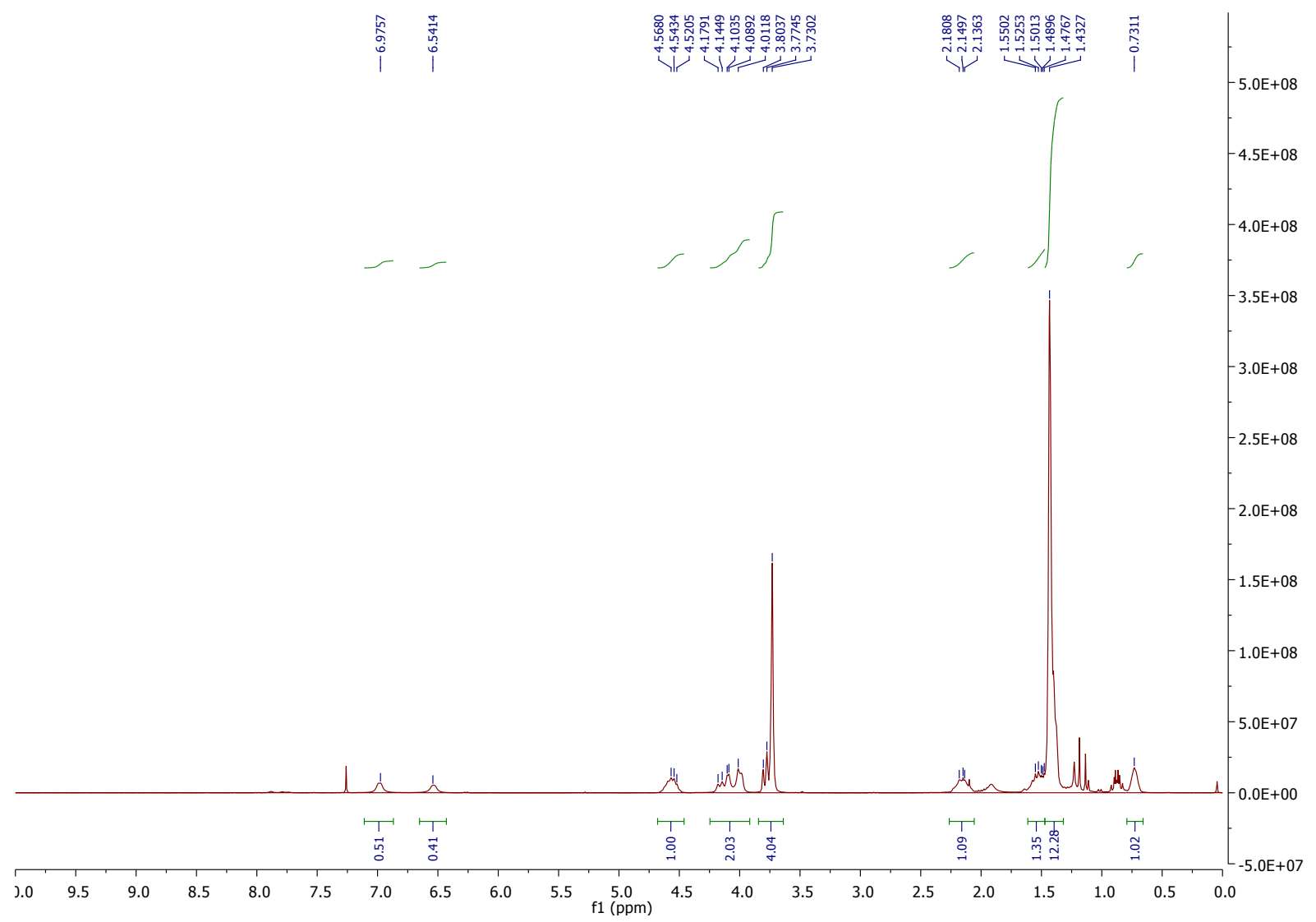


Figure S53. ${ }^{13} \mathrm{C}$ NMR $\{1 \mathrm{H}\}$ spectrum of (-)-10 (75 $\left.\mathrm{MHz}, \mathrm{CDCl}_{3}\right)$.

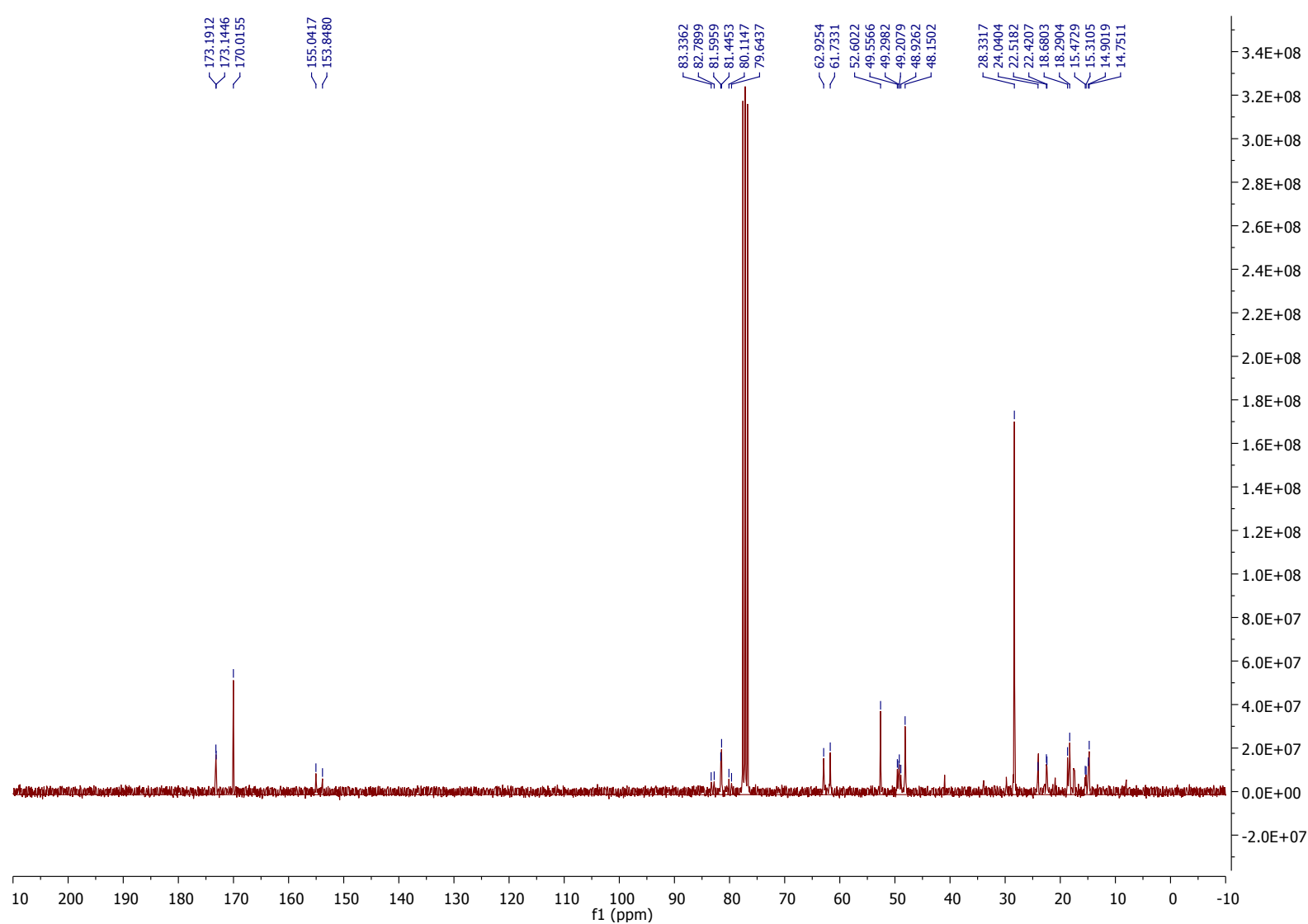

Figure S54. ${ }^{19} \mathrm{~F}$ NMR spectrum of (-)-10 (282 $\left.\mathrm{MHz}, \mathrm{CDCl}_{3}\right)$.

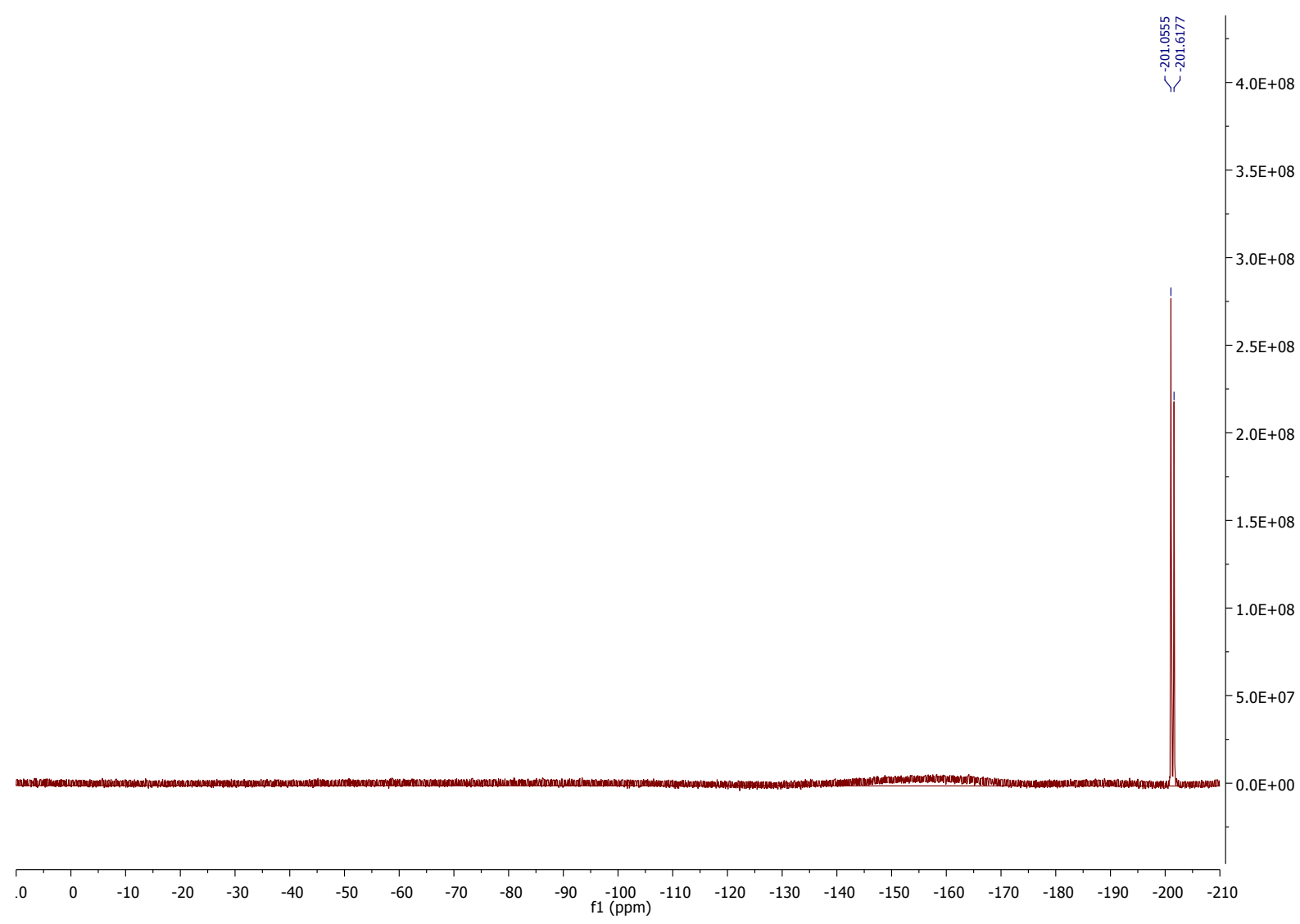


<smiles>O=C1CC(F)CN1Cc1ccc([N+](=O)[O-])cc1</smiles>

(6c) 5-fluoro-3-(4-nitrobenzyl)--3-azabicyclo[3.1.0]hexan-2-one

Figure S55. ${ }^{1} \mathrm{H}$ NMR spectrum of $6 \mathbf{c}\left(300 \mathrm{MHz}, \mathrm{CDCl}_{3}\right)$.

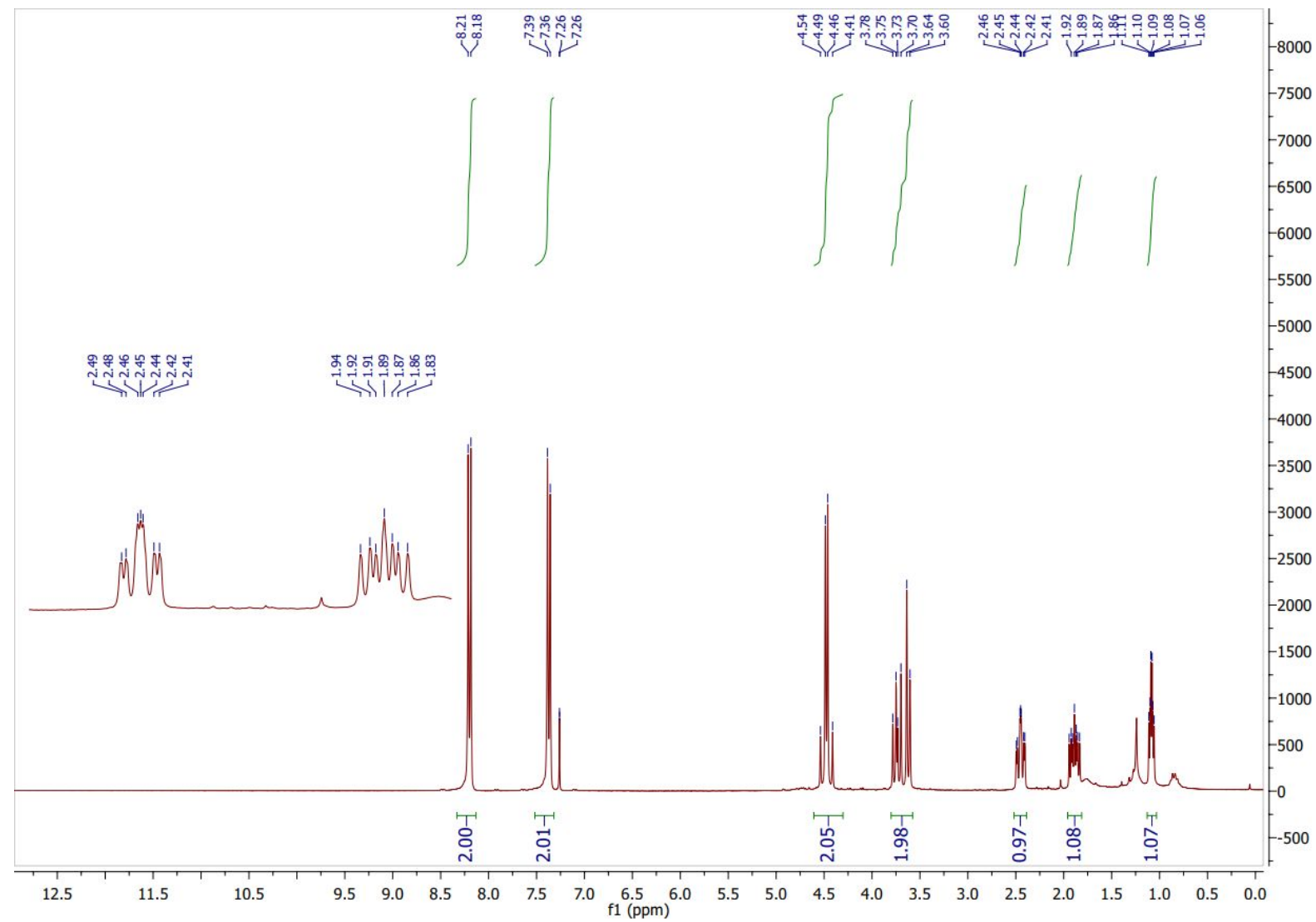


Figure S56. ${ }^{13} \mathrm{C} \mathrm{NMR}\{1 \mathrm{H}\}$ spectrum of $\mathbf{6 c}\left(75 \mathrm{MHz}, \mathrm{CDCl}_{3}\right)$.

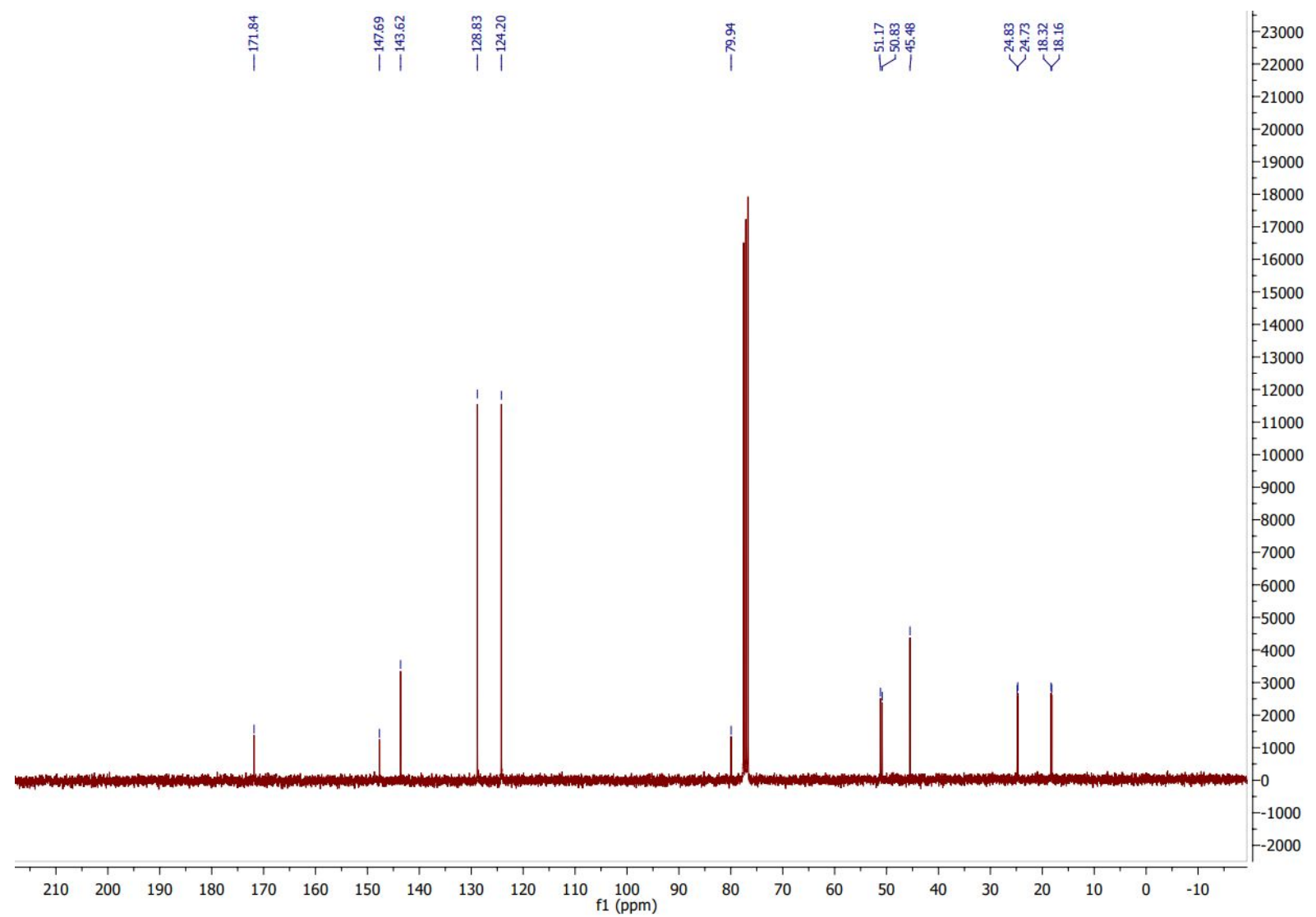


Figure S57. ${ }^{19} \mathrm{~F}$ NMR spectrum of $6 \mathbf{c}\left(282 \mathrm{MHz}, \mathrm{CDCl}_{3}\right)$.

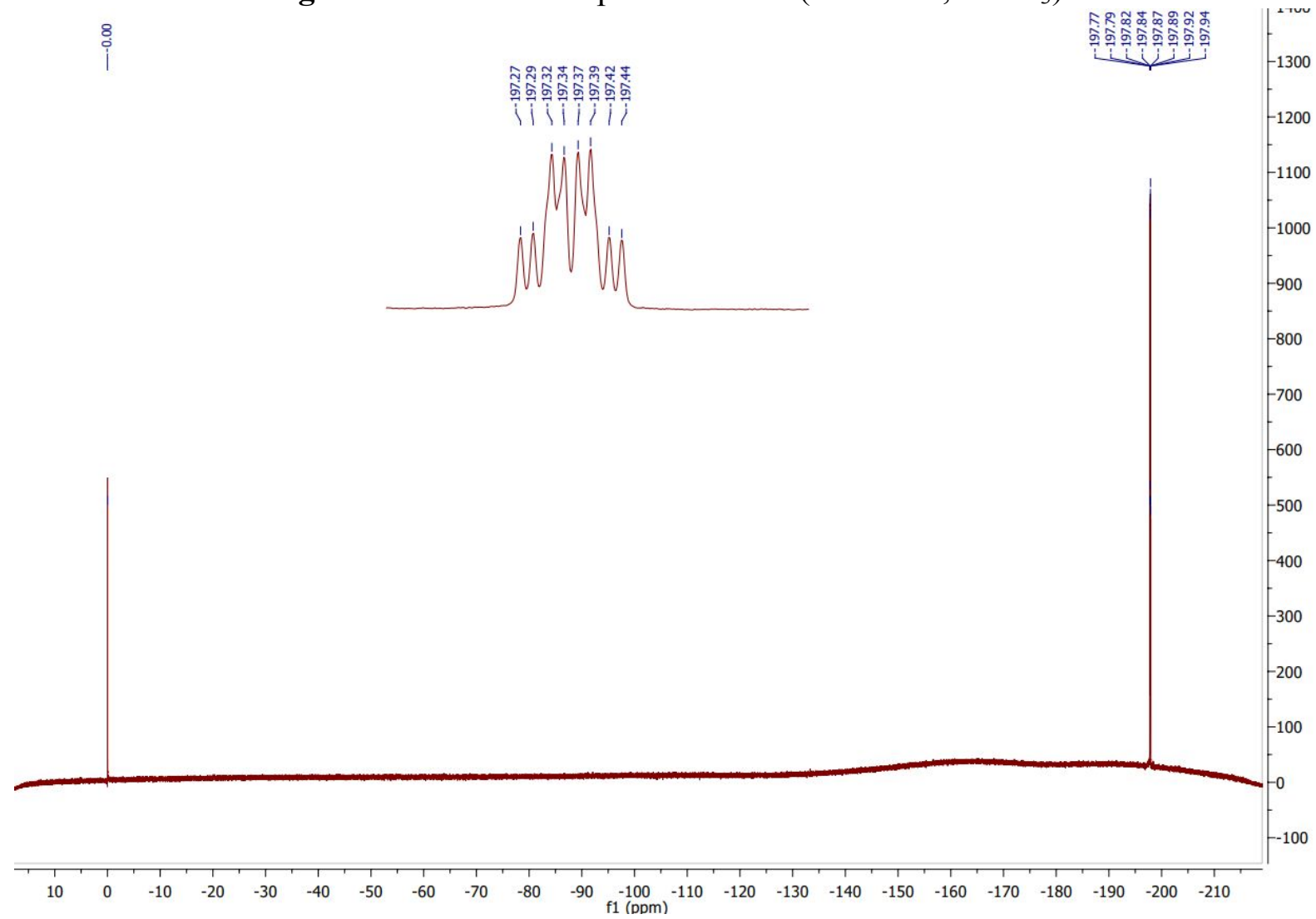

Figure S58. HPLC analysis of $\mathbf{6 c}$ (racemic).

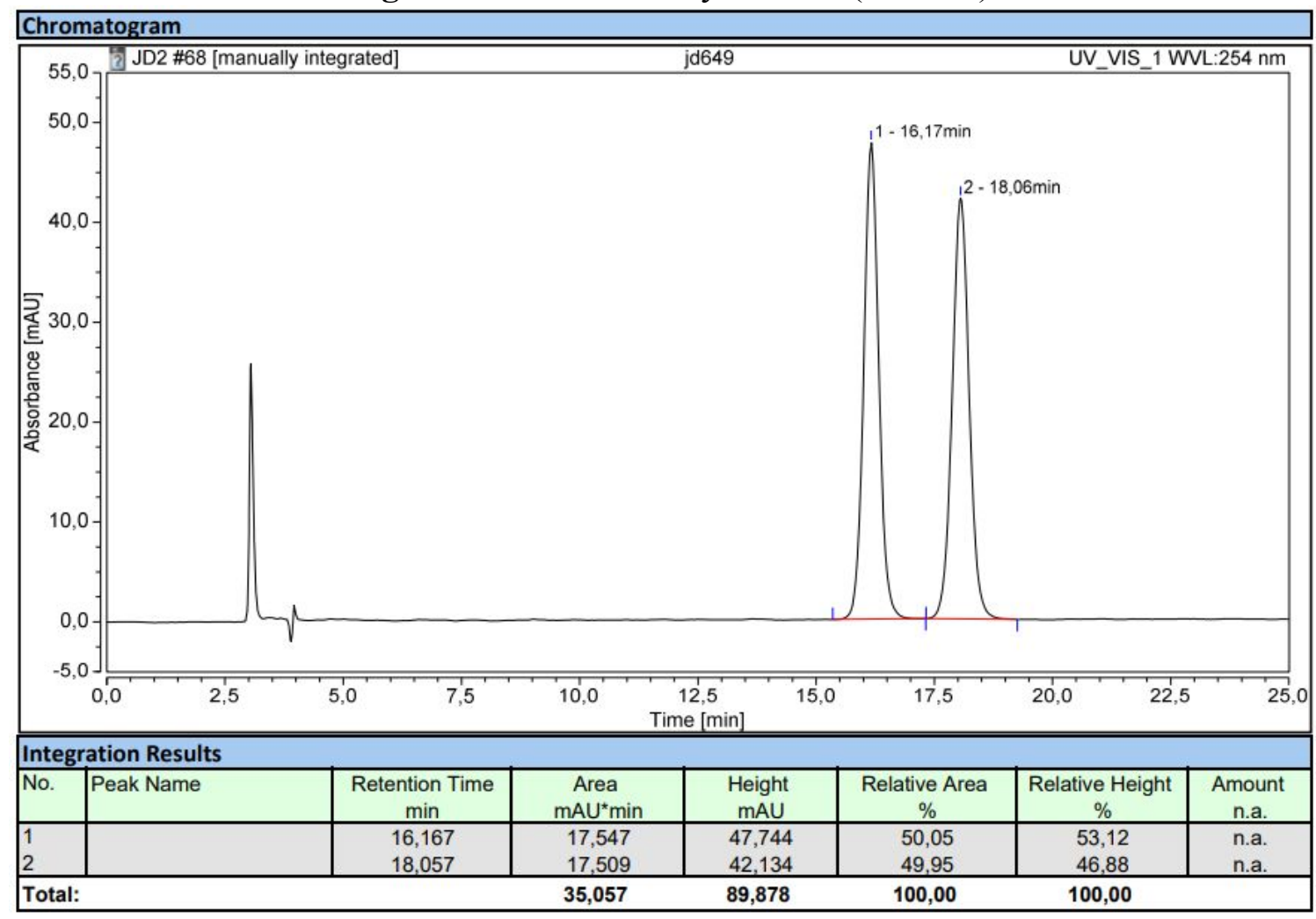


Figure S59. HPLC analysis of $6 \mathbf{c}$ (chiral). ee $=73 \%$

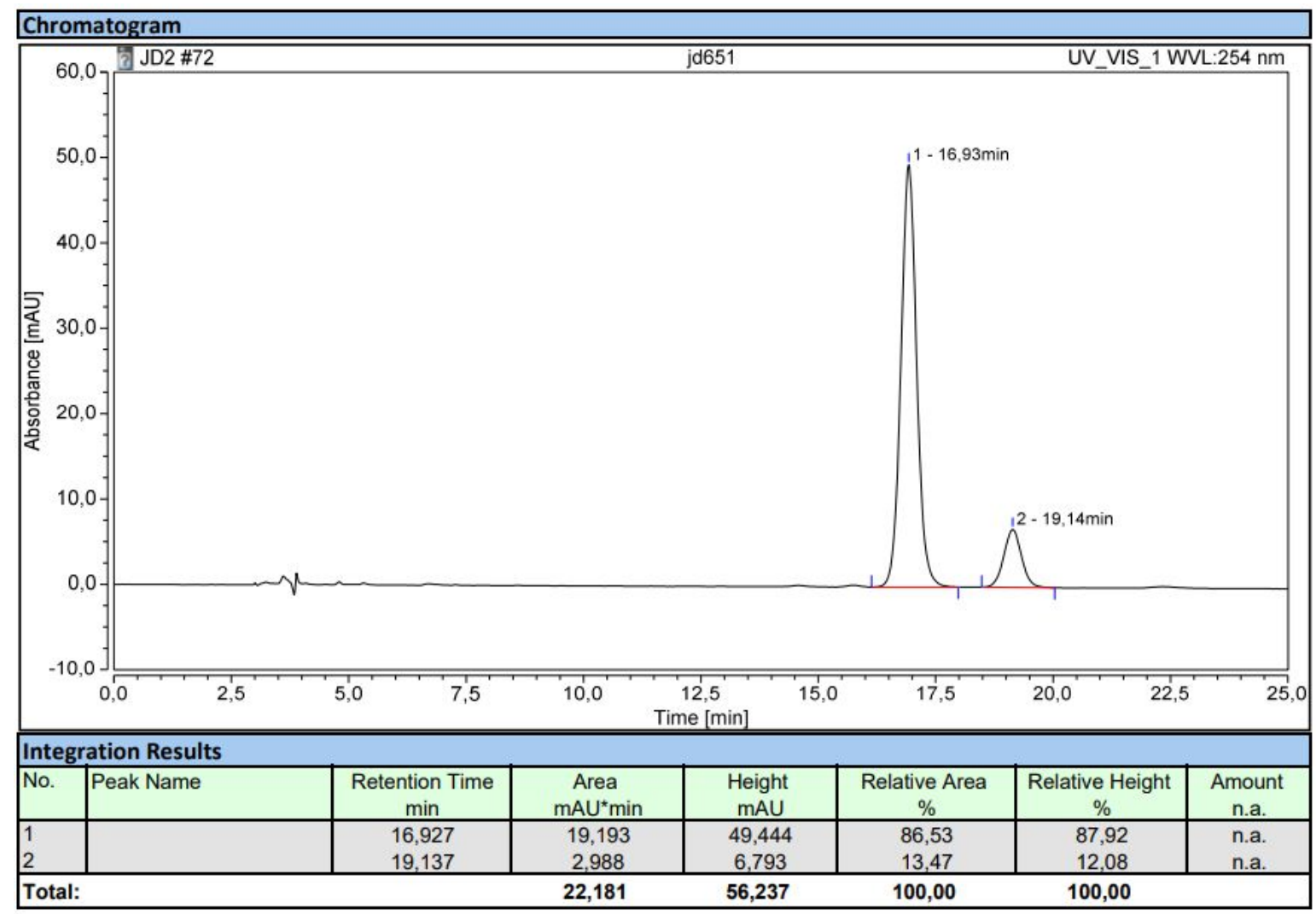


<smiles>O=C1C[C@H](F)CN1Cc1ccc(Cl)cc1</smiles>

(6d) 3-(4-chlorobenzyl)-5-fluoro-3-azabicyclo[3.1.0]hexan-2-one

Figure S60. ${ }^{1} \mathrm{H}$ NMR spectrum of $\mathbf{6 d}\left(300 \mathrm{MHz}, \mathrm{CDCl}_{3}\right)$.

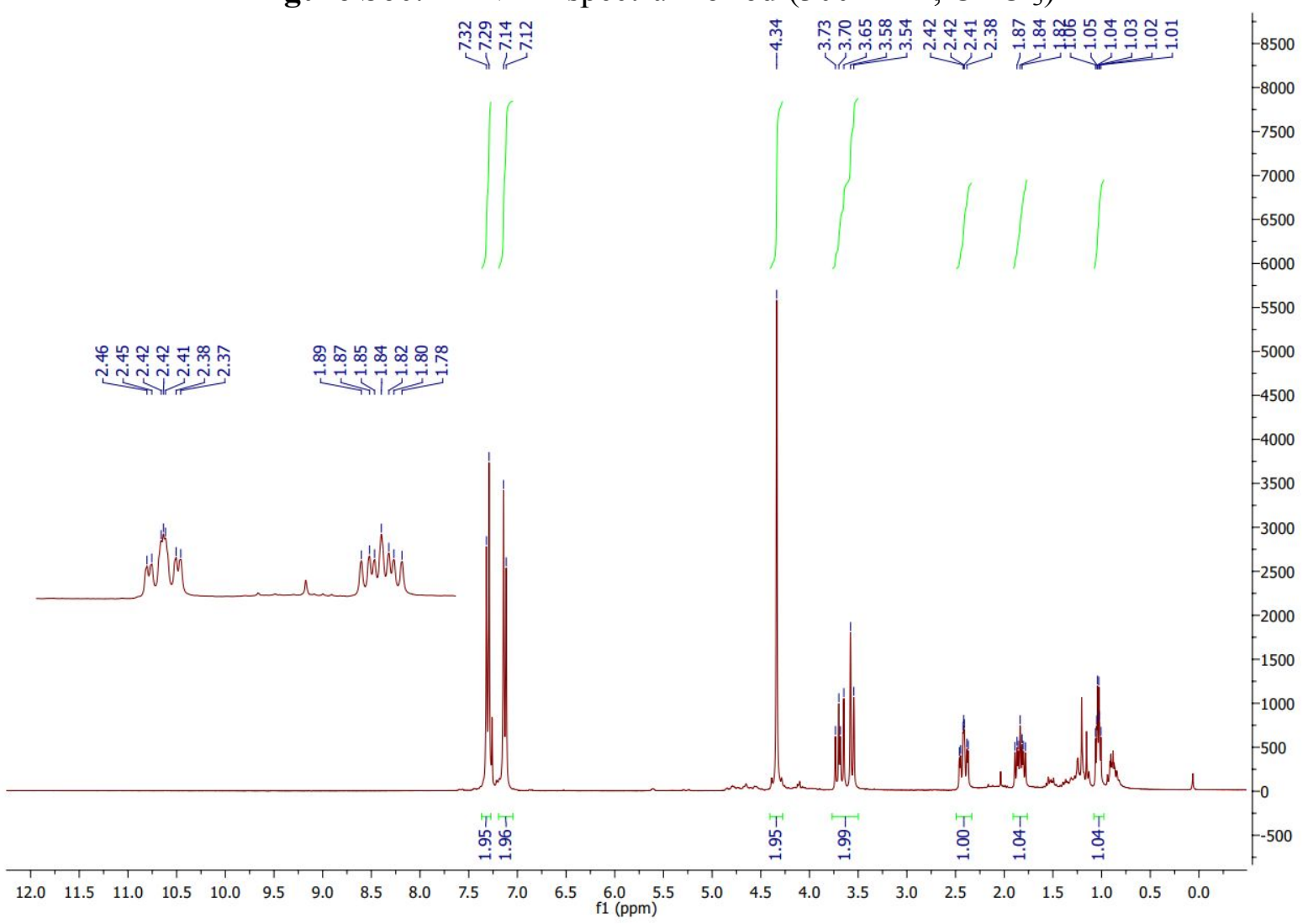


Figure S61. ${ }^{13} \mathrm{C} \mathrm{NMR}\{1 \mathrm{H}\}$ spectrum of $\mathbf{6 d}\left(75 \mathrm{MHz}, \mathrm{CDCl}_{3}\right)$.

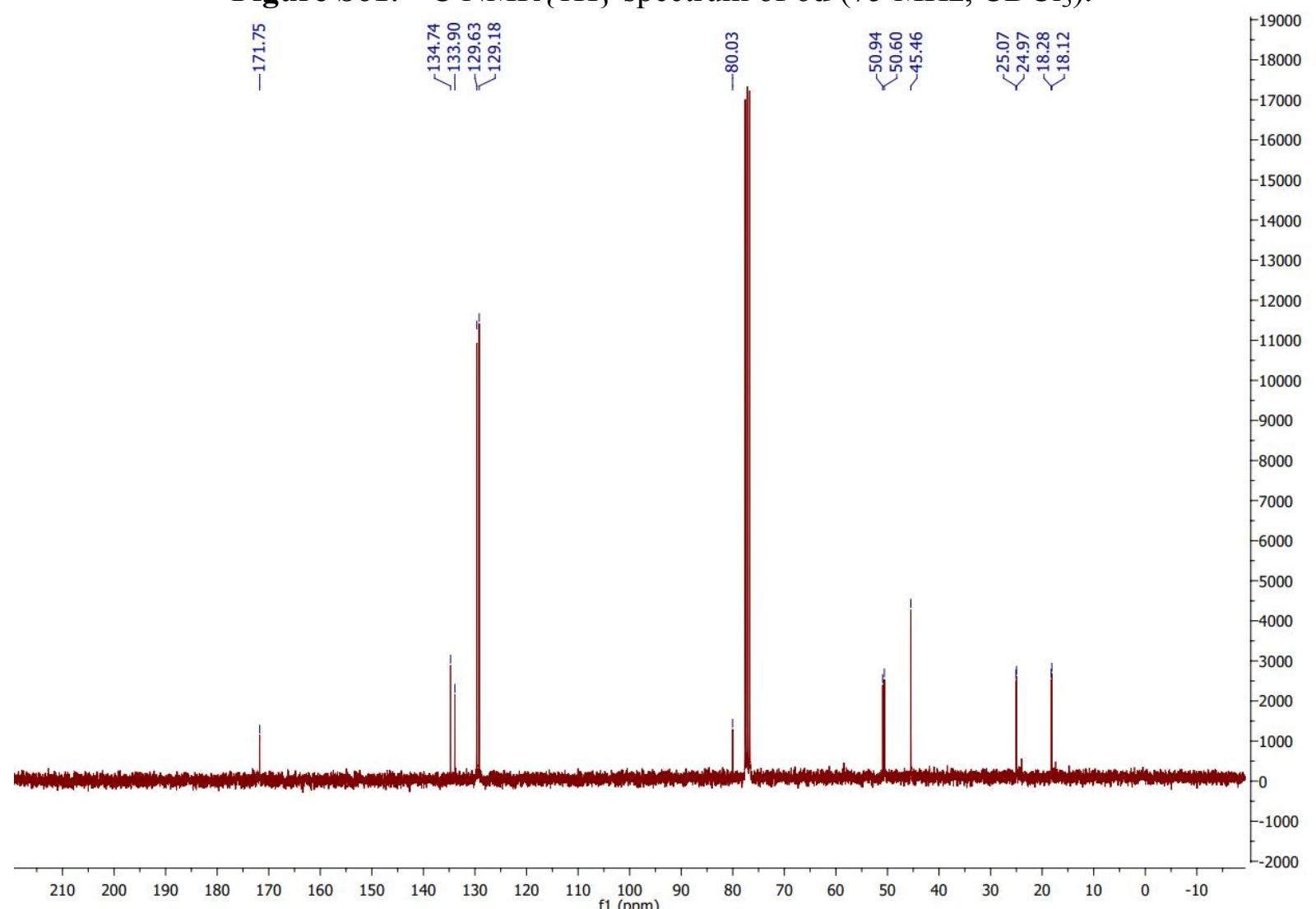

Figure S62. ${ }^{19} \mathrm{~F}$ NMR spectrum of $\mathbf{6 d}\left(282 \mathrm{MHz}, \mathrm{CDCl}_{3}\right)$.

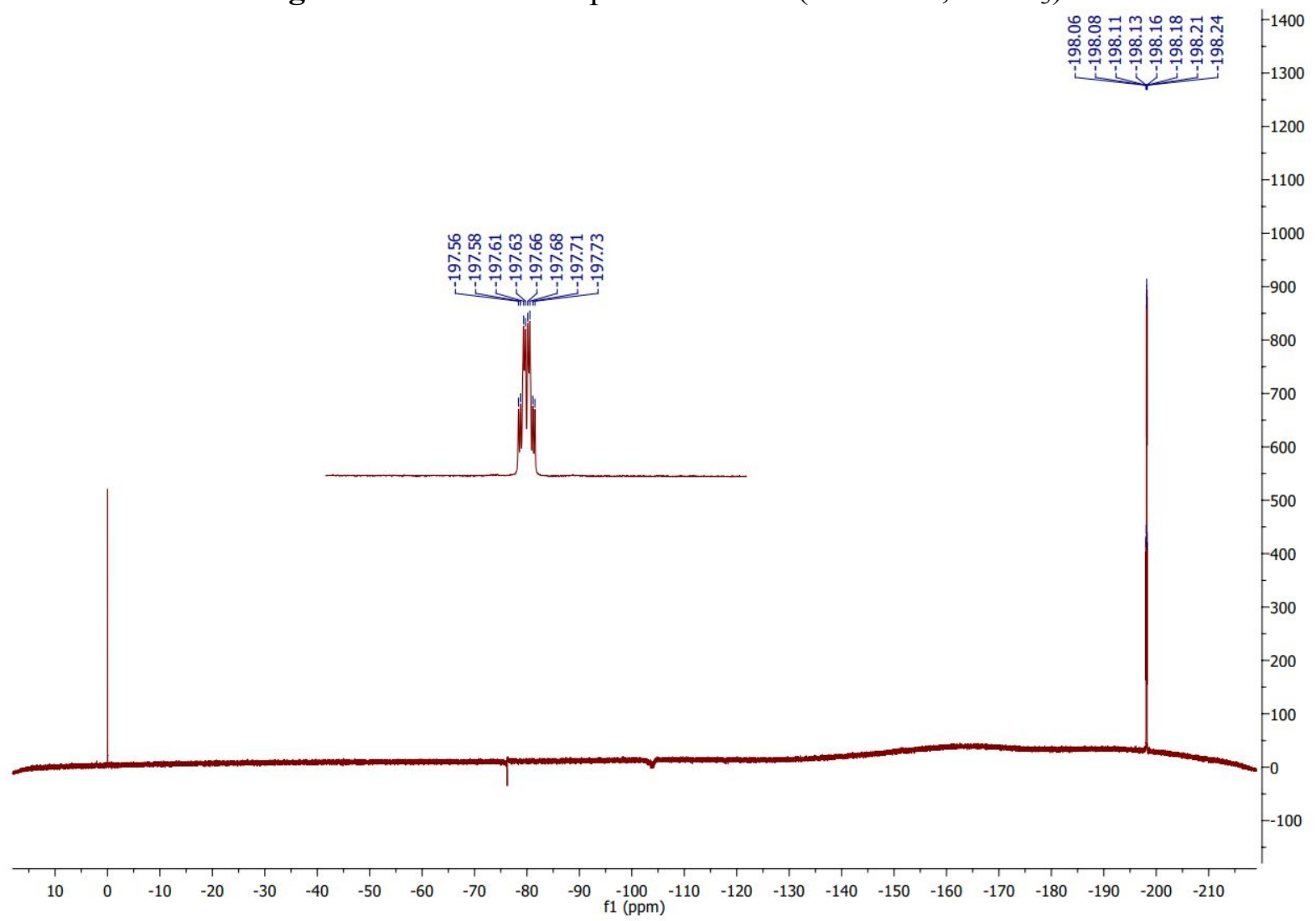


Figure S63. HPLC analysis of 6d (racémic).

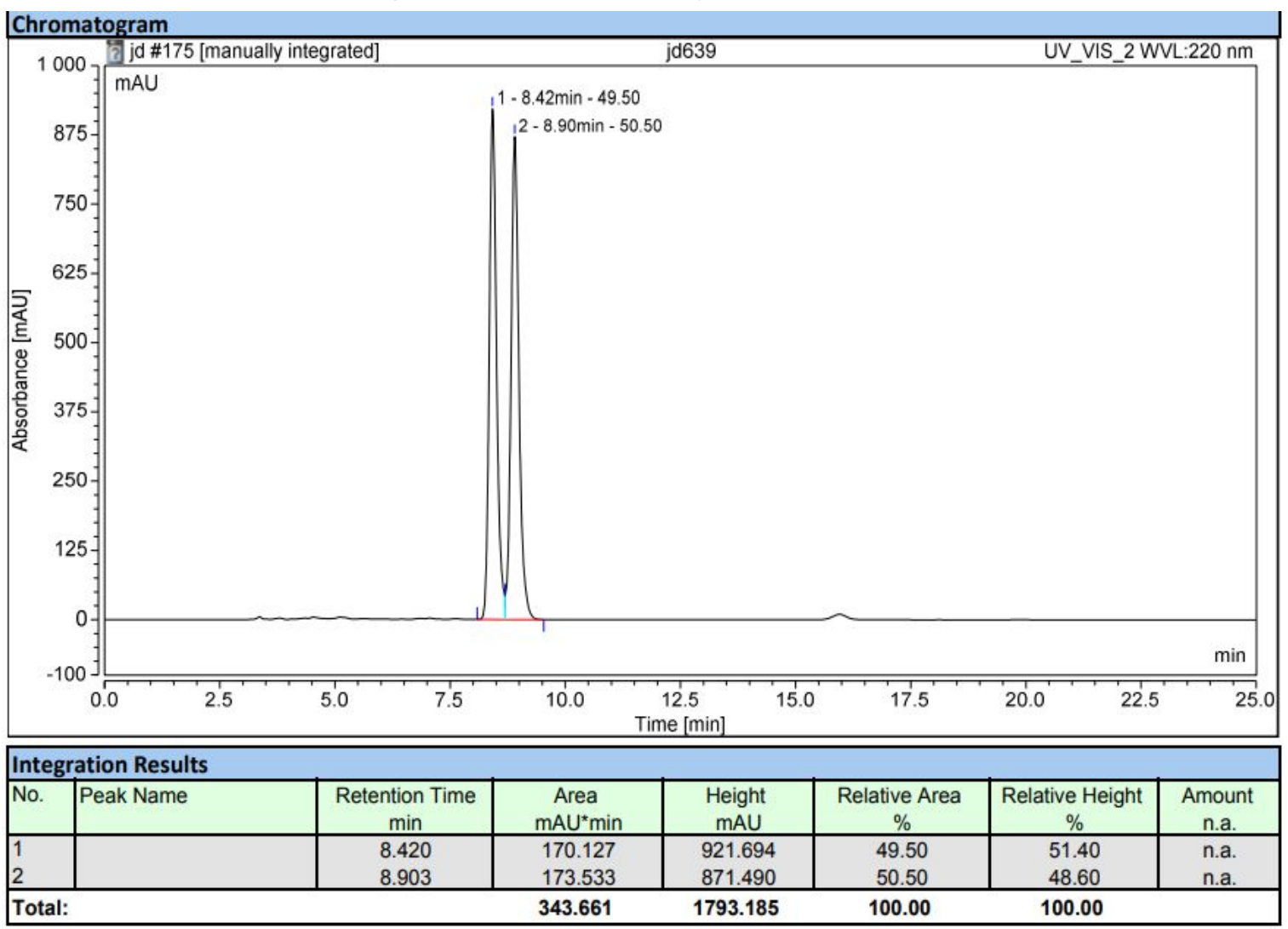

Figure S64. HPLC analysis of $\mathbf{6 d}$ (chiral). ee $=70 \%$

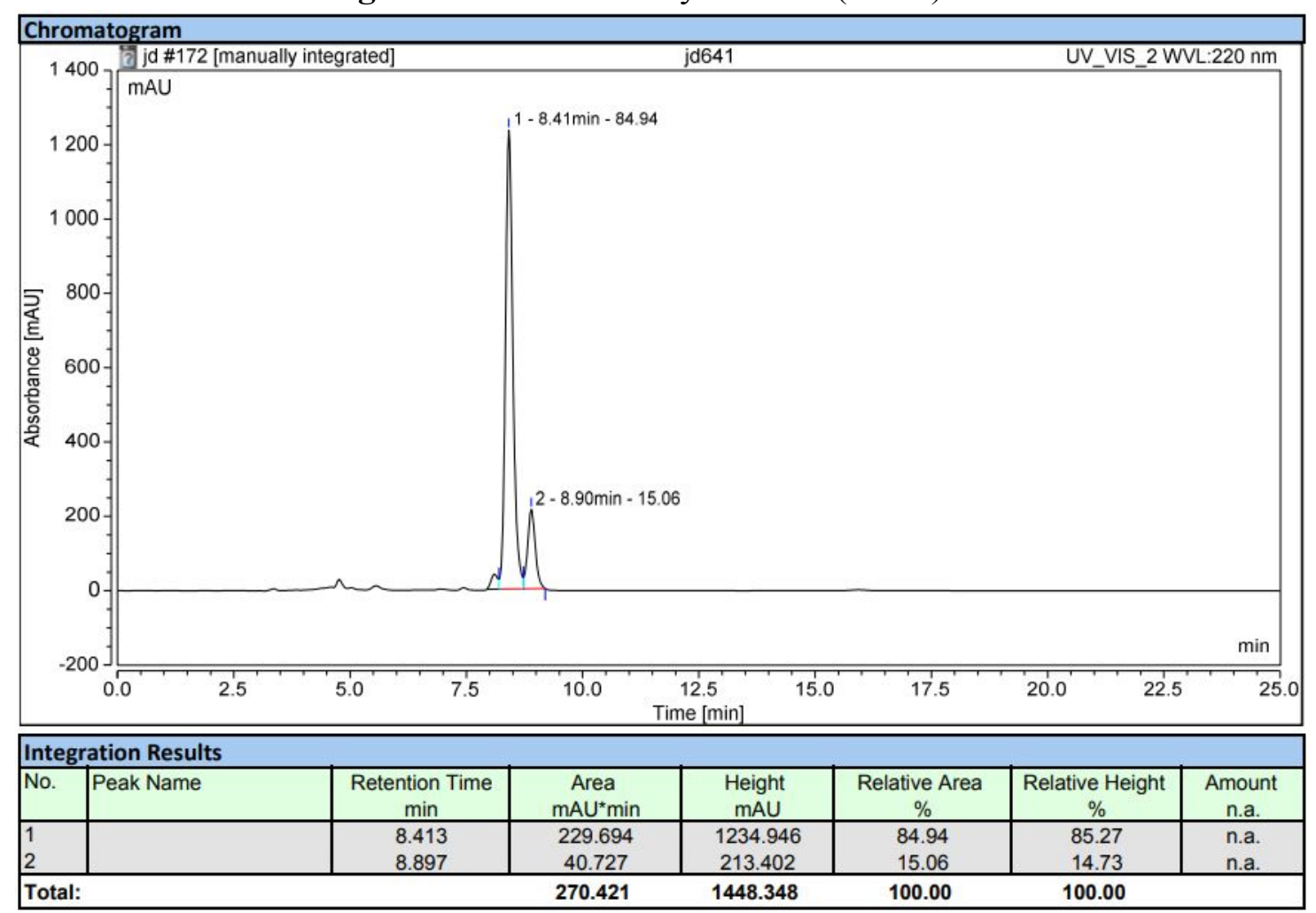


Structural determination of ((-)-11) (S)-methyl 2-((1R,2S,5S)-3-((S)-2-((tertbutoxycarbonyl)amino)propanoyl)-5-fluoro-3-azabicyclo[3.1.0]hexane-2carboxamido)propanoate

Figure S65. ${ }^{19} \mathrm{~F}$ NMR spectrum of (-)-11 obtained without (a) and with (b) proton decoupling during acquisition $\left(\mathrm{CDCl}_{3}, 470 \mathrm{MHz}, 298 \mathrm{~K}\right)$.

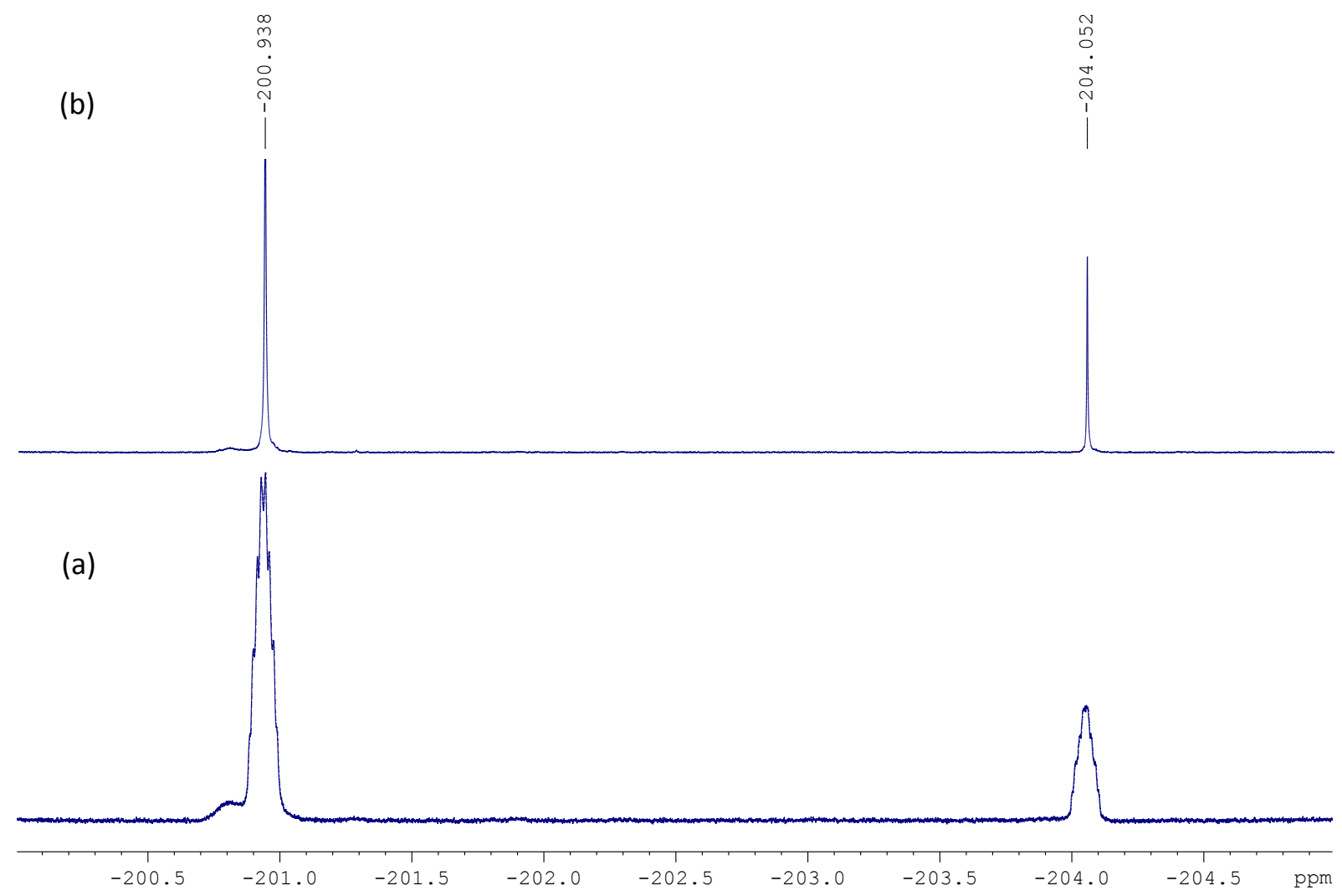


Figure S66. ${ }^{13} \mathrm{C}$ with $J$-modulation of spin-echo (a) and ${ }^{13} \mathrm{C}$ (b) NMR spectra of (-)-11 $\left(\mathrm{CDCl}_{3}\right.$, $125 \mathrm{MHz}, 298 \mathrm{~K})$.

(a)

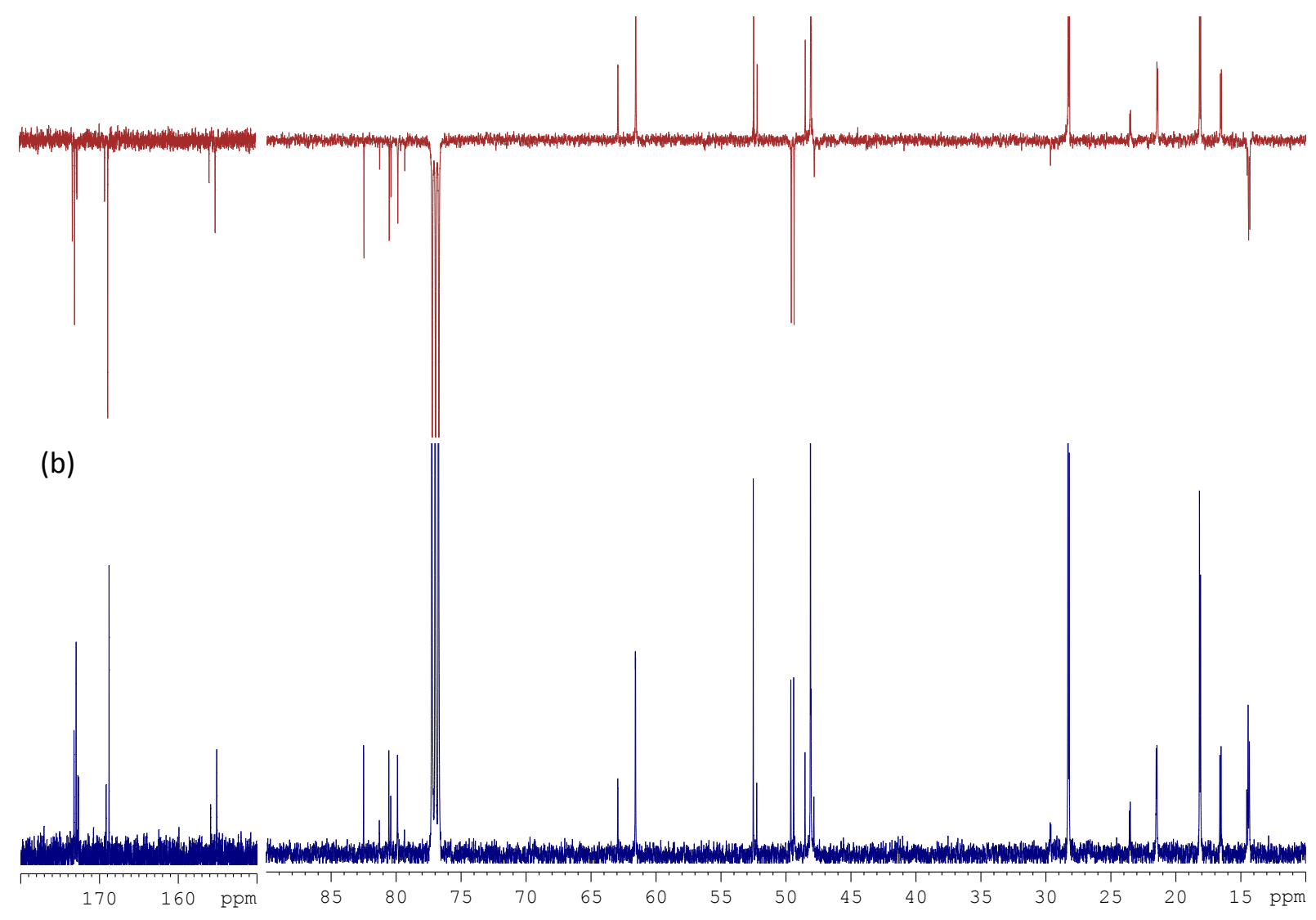


Figure S67. ${ }^{1} \mathrm{H}$ NMR spectrum of (-)-11 obtained without fluorine decoupling during acquisition $\left(\mathrm{CDCl}_{3}, 500 \mathrm{MHz}, 298 \mathrm{~K}\right)$.

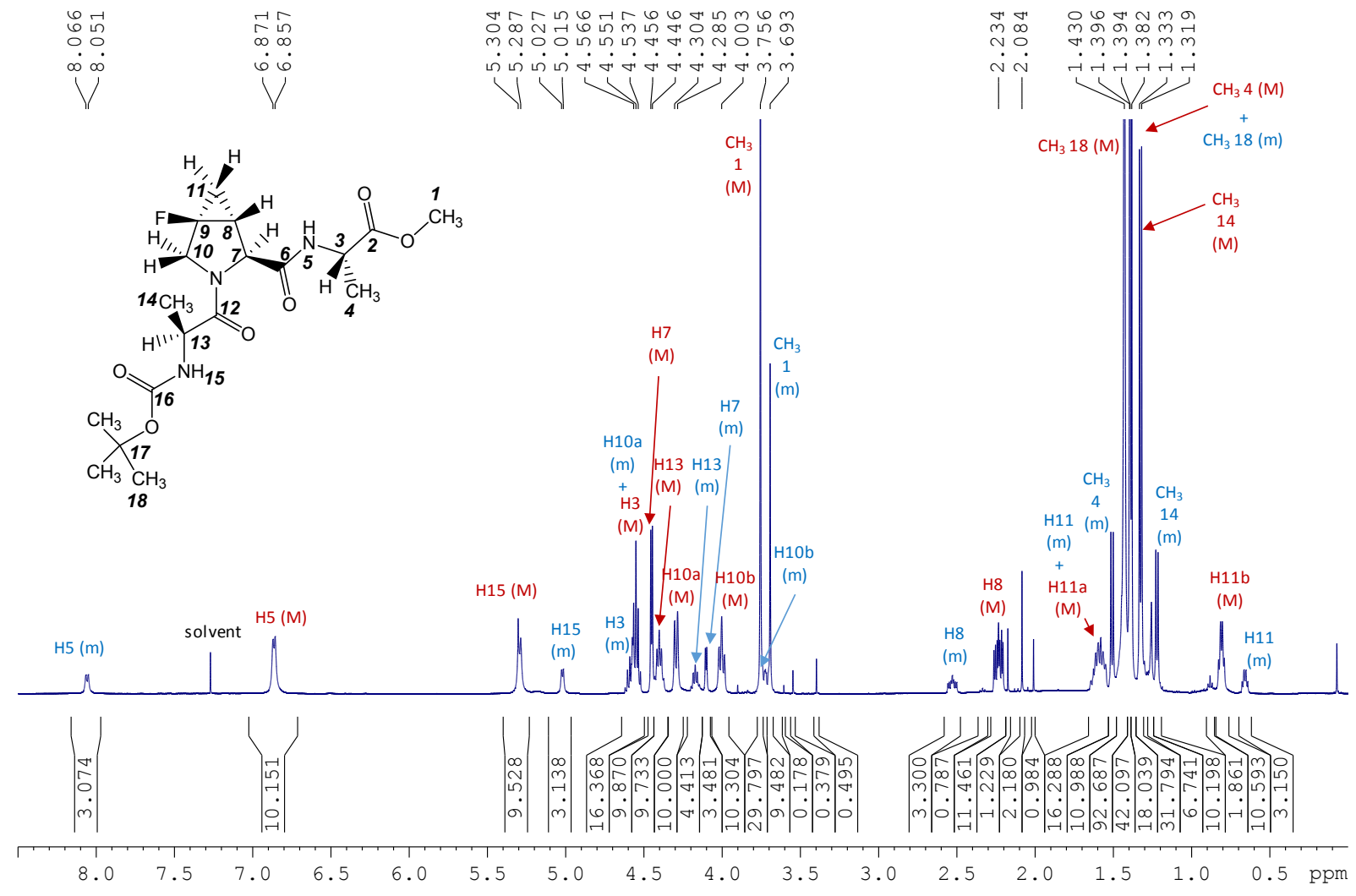


Figure S68. Zooms of ${ }^{1} \mathrm{H}$ NMR spectra (a) without and with (b) fluorine decoupling during acquisition and (c) ${ }^{1} \mathrm{H}^{-19} \mathrm{~F}$ HOE NMR spectrum of (-)-11 $\left.\mathrm{CDCl}_{3}, 500 \mathrm{MHz}, 298 \mathrm{~K}\right)$.

(c)

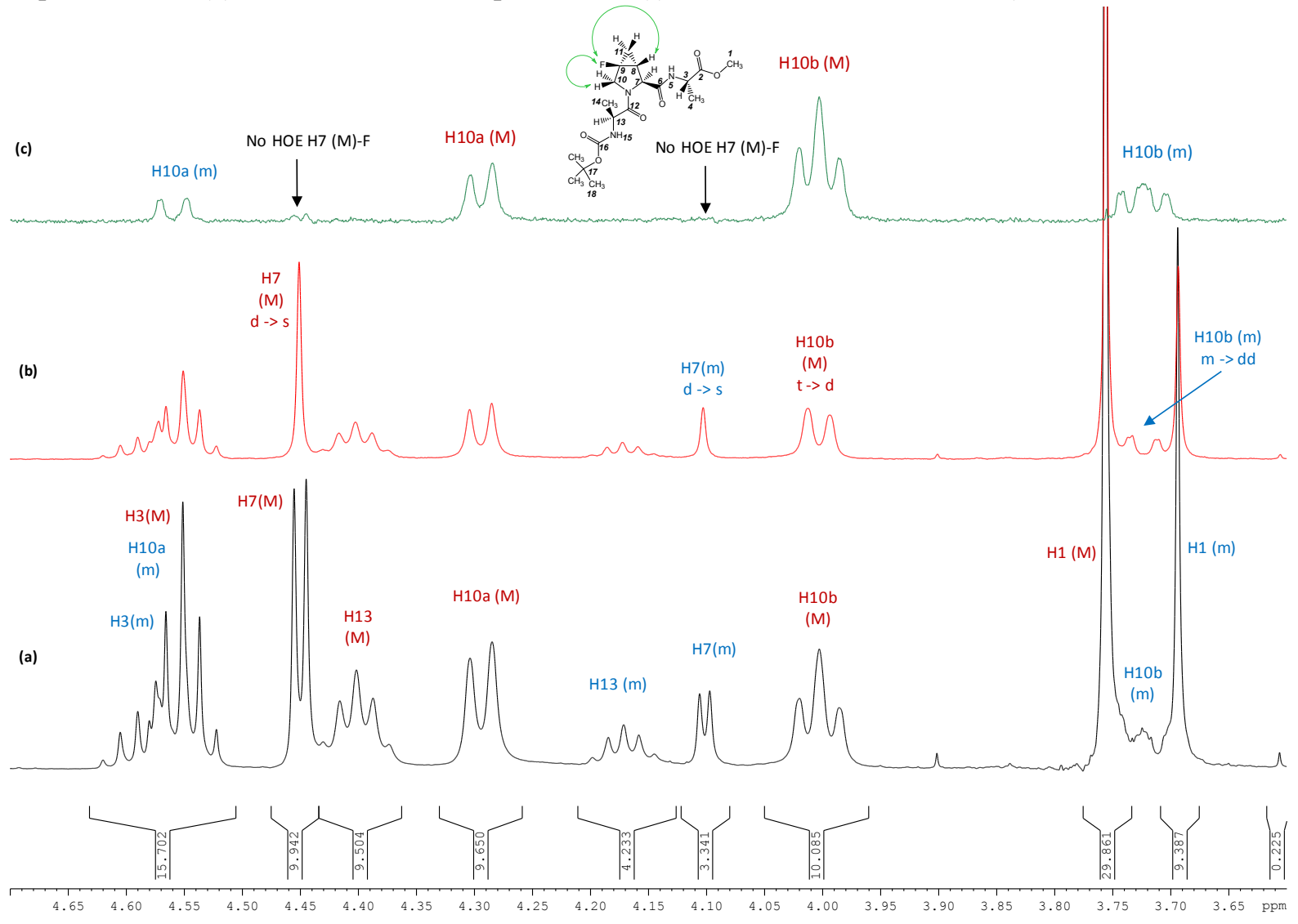

S53 
Figure S69. Zooms of ${ }^{1} \mathrm{H}$ NMR spectra (a) without and with (b) fluorine decoupling during acquisition and (c) ${ }^{1} \mathrm{H}-{ }^{19} \mathrm{~F}$ HOE NMR spectrum of (-)-11 $\left.\mathrm{CDCl}_{3}, 500 \mathrm{MHz}, 298 \mathrm{~K}\right)$.

(c)

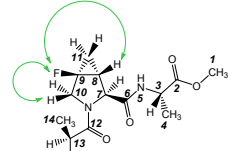

H8 (M)

${ }^{\circ} \int_{16}^{\mathrm{NH}_{15}}$

$\mathrm{H} 8(\mathrm{~m})$

(b)

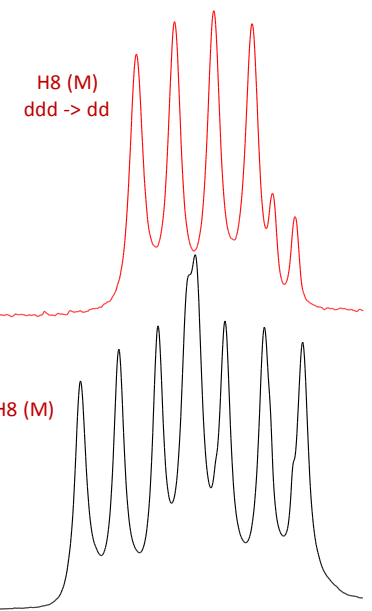

(a)

H8 (m)

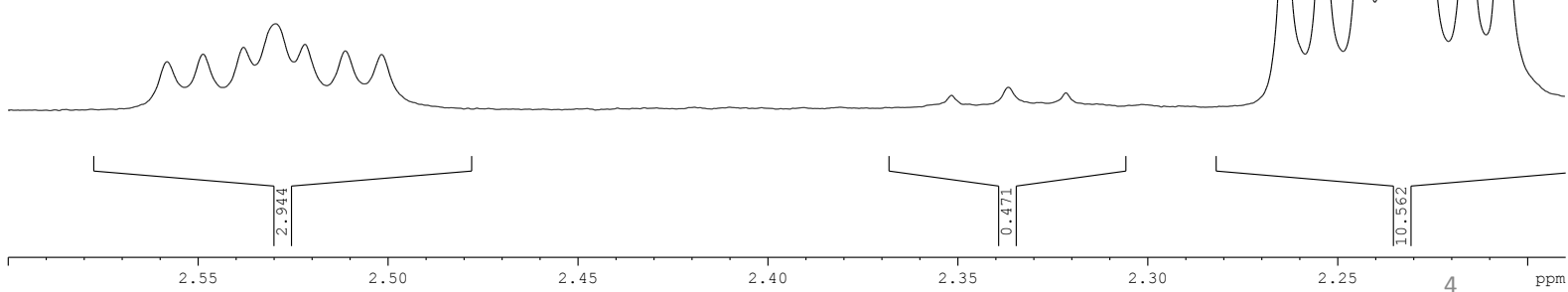


Figure S70. Zooms of ${ }^{1} \mathrm{H}$ NMR spectra (a) without and with (b) fluorine decoupling during acquisition and (c) ${ }^{1} \mathrm{H}_{-}{ }^{19} \mathrm{~F}$ HOE NMR spectrum of (-)-11 $\left.\mathrm{CDCl}_{3}, 500 \mathrm{MHz}, 298 \mathrm{~K}\right)$.

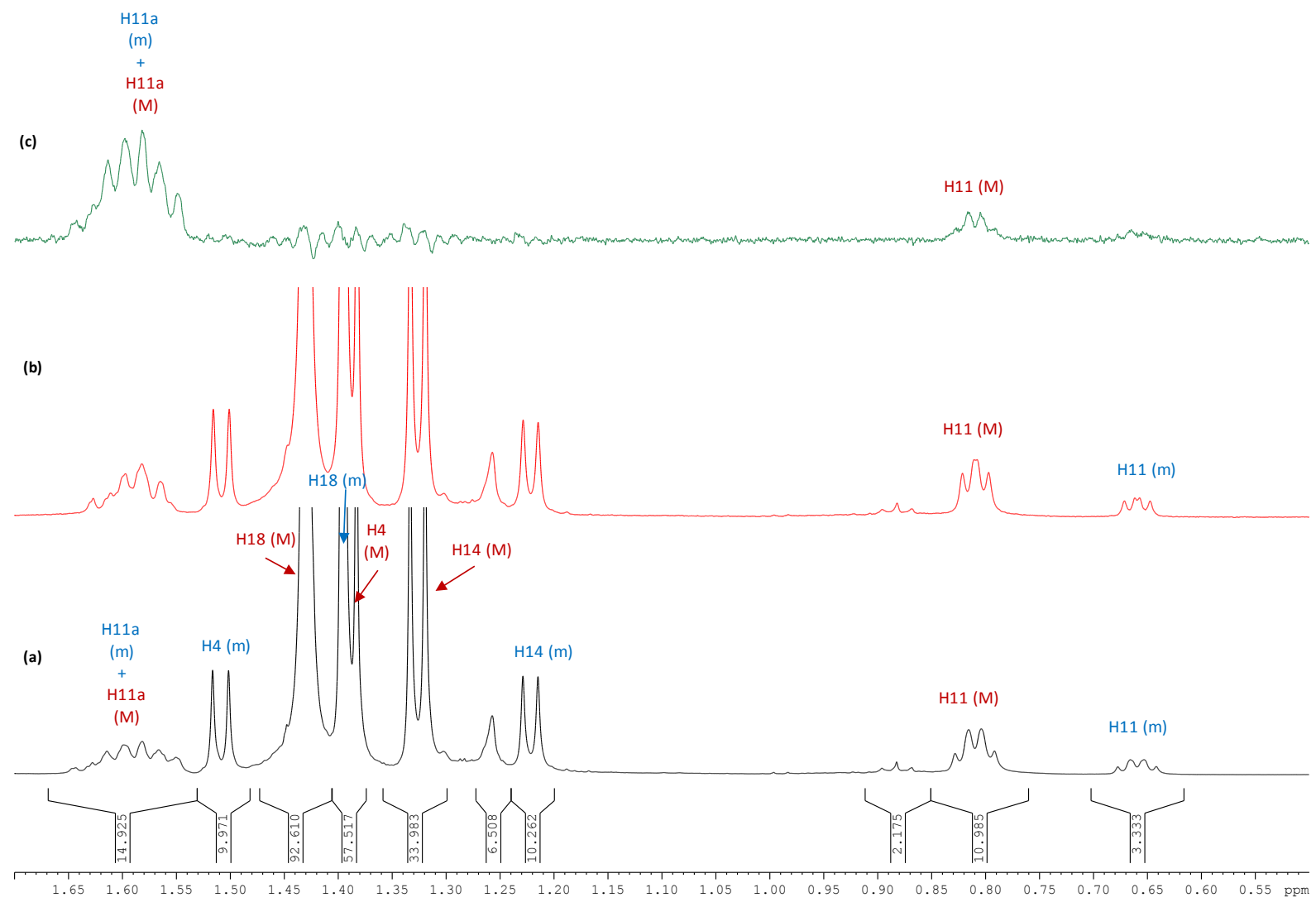


Figure S71. ${ }^{1} \mathrm{H}-{ }^{1} \mathrm{H}$ COSY NMR spectrum of (-)-11 $\left(\mathrm{CDCl}_{3}, 500 \mathrm{MHz}, 298 \mathrm{~K}\right)$.

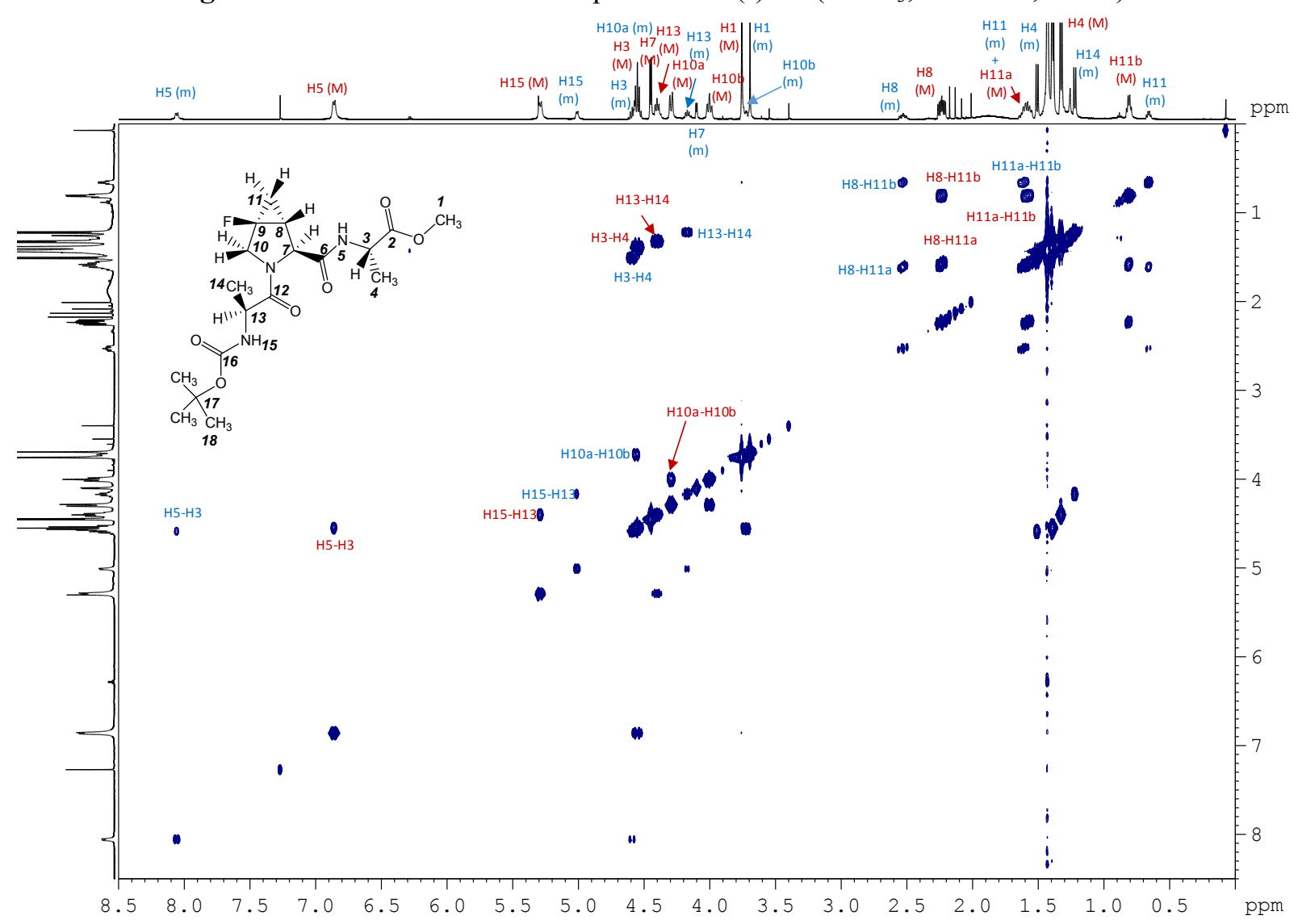


Figure S72. ${ }^{1} \mathrm{H}-{ }^{1} \mathrm{H}$ NOESY NMR spectrum of (-)-11 $\left(\mathrm{CDCl}_{3}, 500 \mathrm{MHz}, 298 \mathrm{~K}\right)$. Blue correlations are due to NOE whereas red ones are due to conformational exchange.

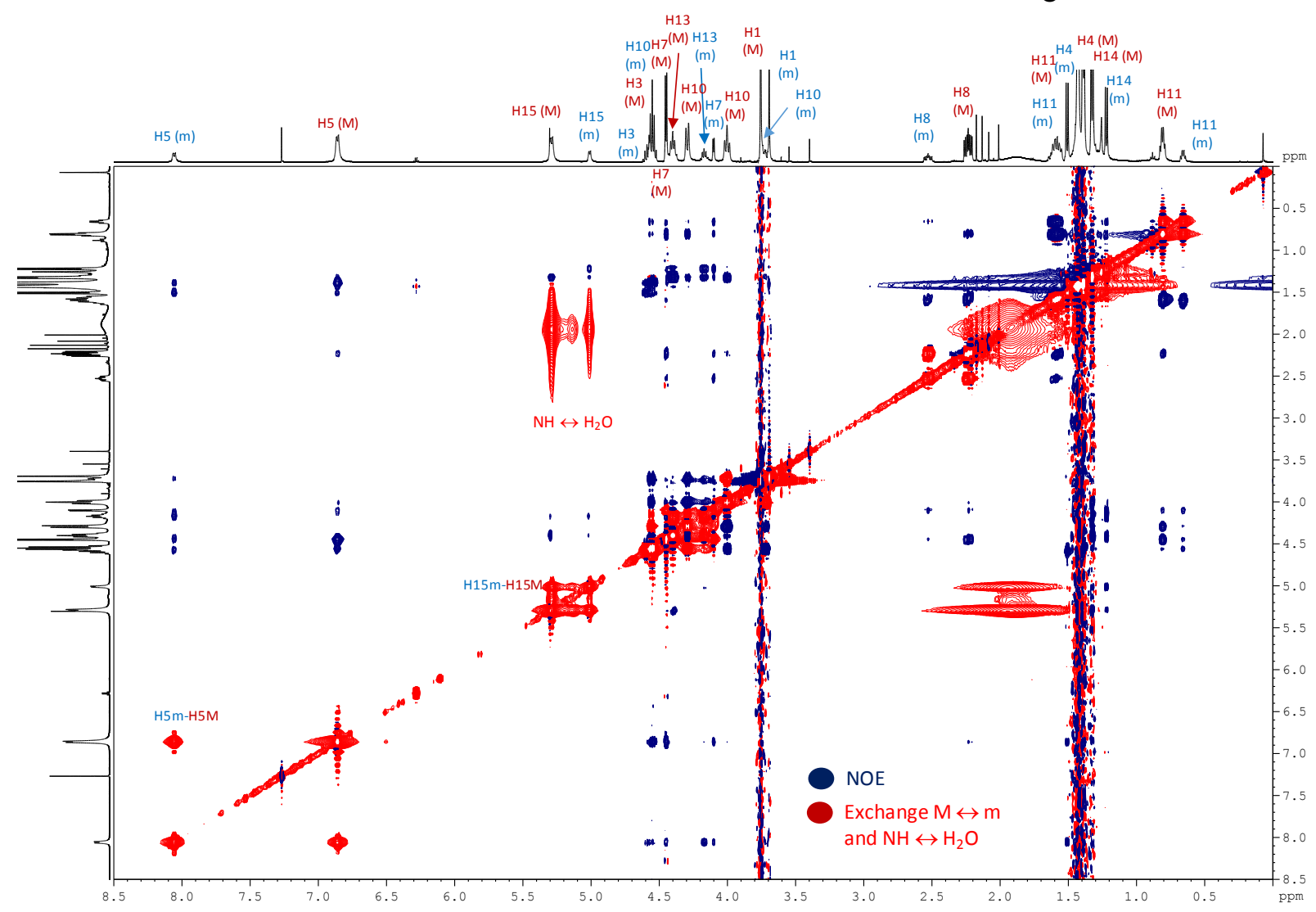


Figure S73a. ${ }^{1} \mathrm{H}-{ }^{1} \mathrm{H}$ NOESY NMR spectrum of (-)-11 ( $\left.\mathrm{CDCl}_{3}, 600 \mathrm{MHz}, 275 \mathrm{~K}\right)$. Blue correlations are due to NOE whereas red ones are due to conformational exchange.

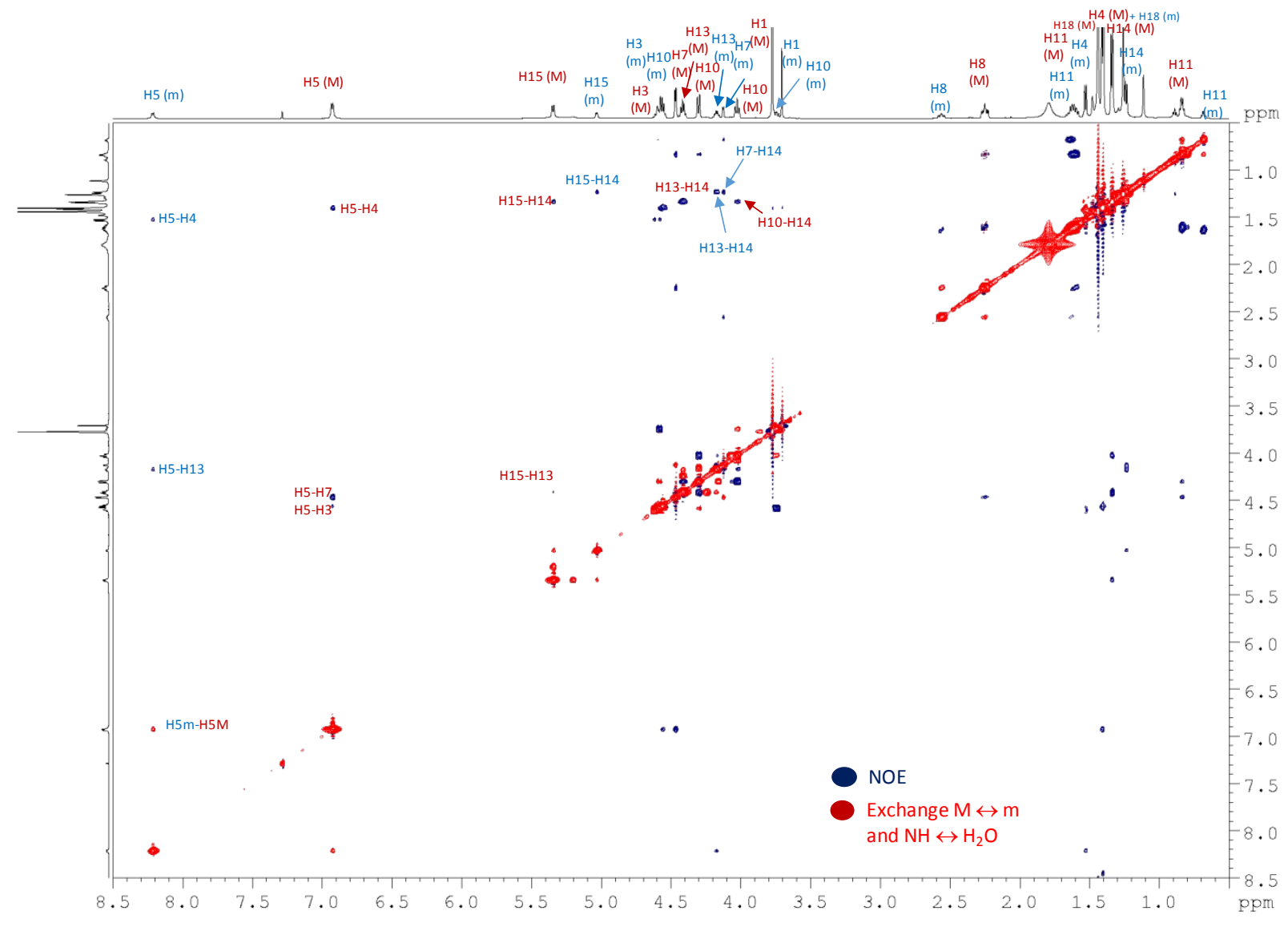


Figure S73b. Zoom of ${ }^{1} \mathrm{H}-{ }^{-1} \mathrm{H}$ NOESY NMR spectrum of (-)-11 $\left(\mathrm{CDCl}_{3}, 600 \mathrm{MHz}, 275 \mathrm{~K}\right)$.

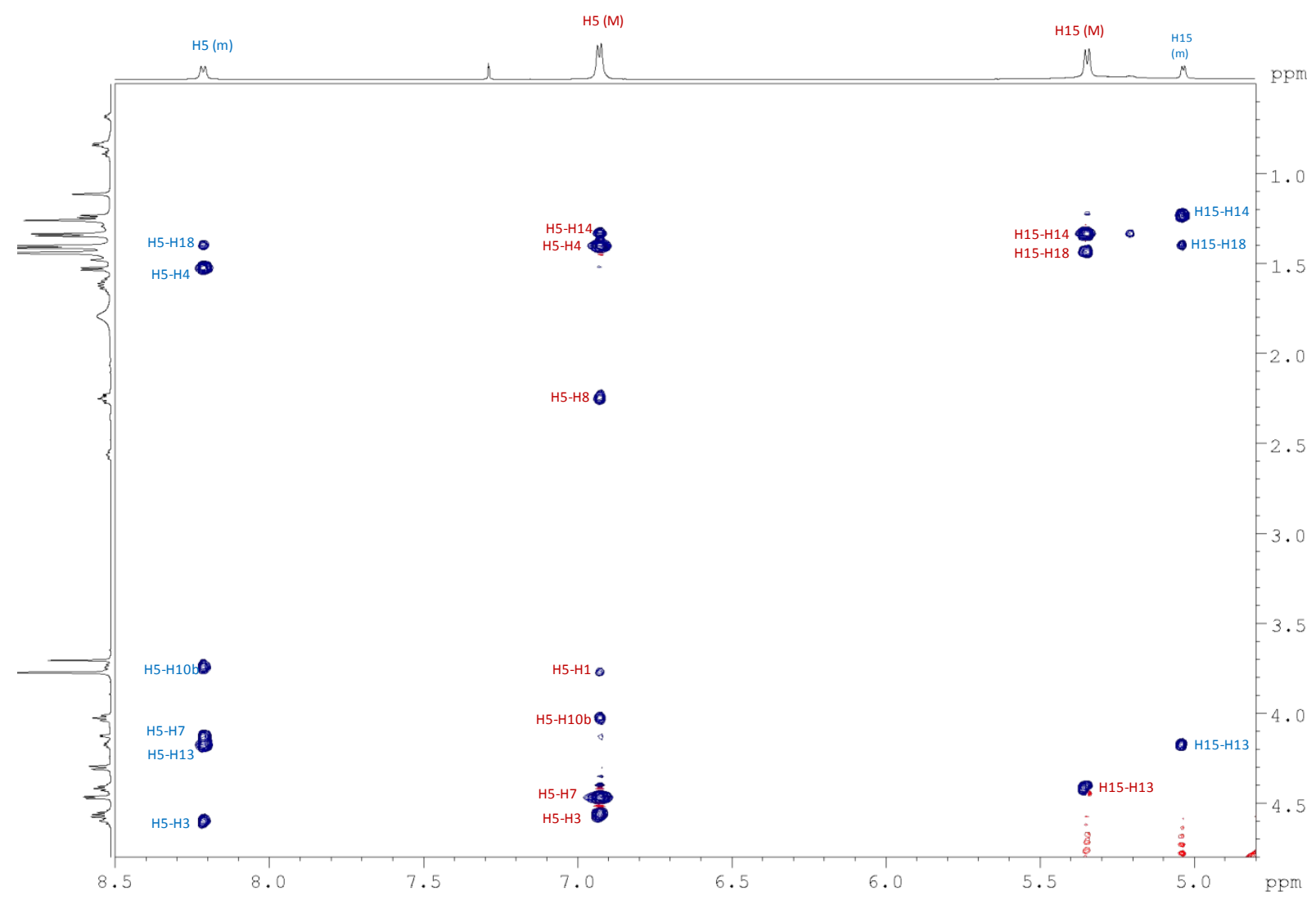


Figure S73c. Zoom of ${ }^{1} \mathrm{H}-{ }^{1} \mathrm{H}$ NOESY NMR spectrum of (-)-11 $\left(\mathrm{CDCl}_{3}, 600 \mathrm{MHz}, 275 \mathrm{~K}\right)$.

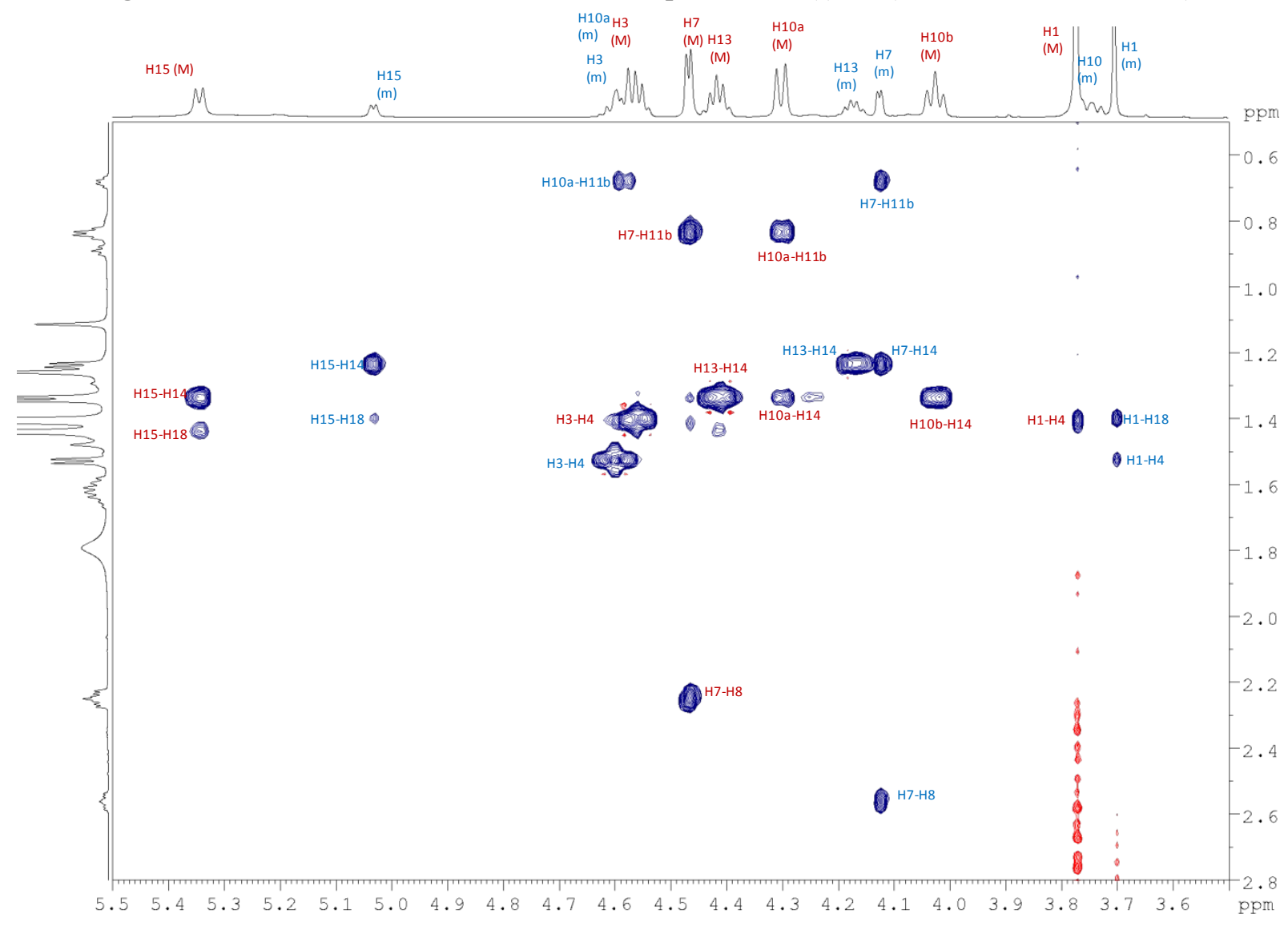


Figure S74. ${ }^{1} \mathrm{H}-{ }^{13} \mathrm{C}$ HMQC NMR spectrum of $(-)-11\left(\mathrm{CDCl}_{3}, 500 \mathrm{MHz}, 298 \mathrm{~K}\right)$.

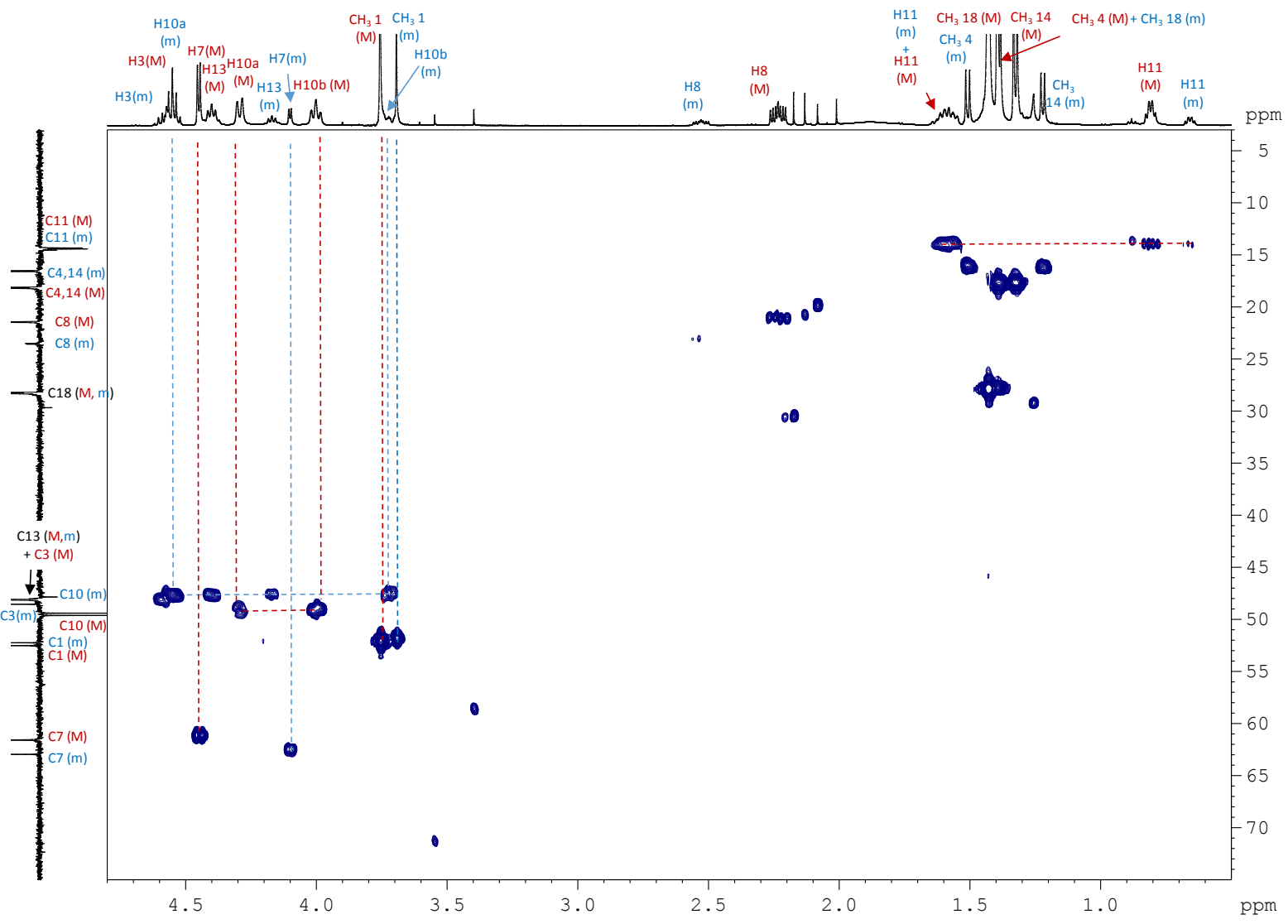


Figure S75. Zoom of ${ }^{1} \mathrm{H}-{ }^{13} \mathrm{C}$ HMBC NMR spectrum of (-)-11 $\left(\mathrm{CDCl}_{3}, 500 \mathrm{MHz}, 298 \mathrm{~K}\right)$.

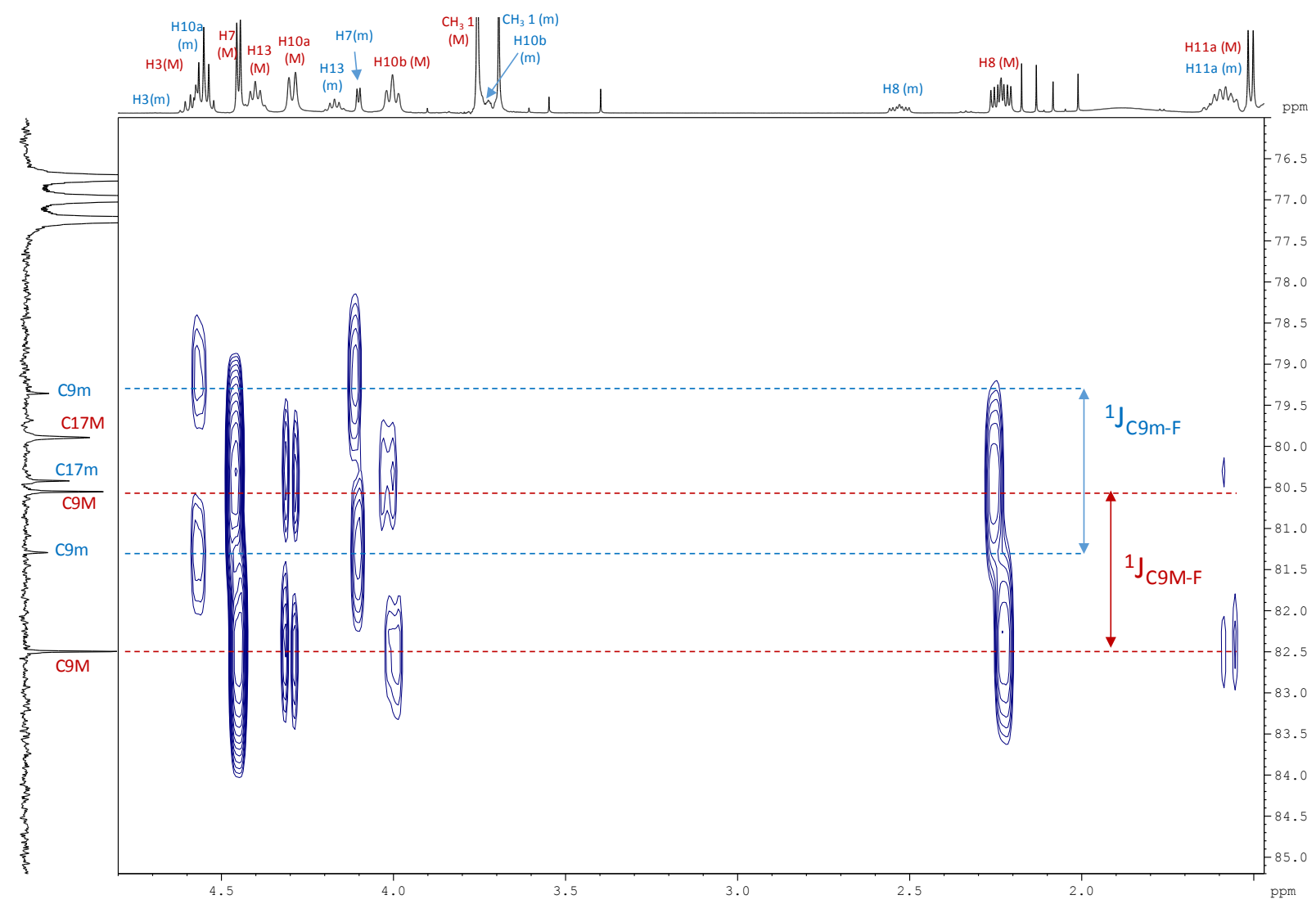


Figure S76. ${ }^{1} \mathrm{H}$ NMR spectra (expansion of the N-H zone) of (-)-11 (500MHz, 298K), in $\mathrm{CDCl}_{3}$ (a) and with addition of $\mathrm{D}_{2} \mathrm{O}$ at $\mathrm{t}=5 \mathrm{~min}(\mathrm{~b})$ and $\mathrm{t}=12 \mathrm{~h}(\mathrm{c})$.

(c)

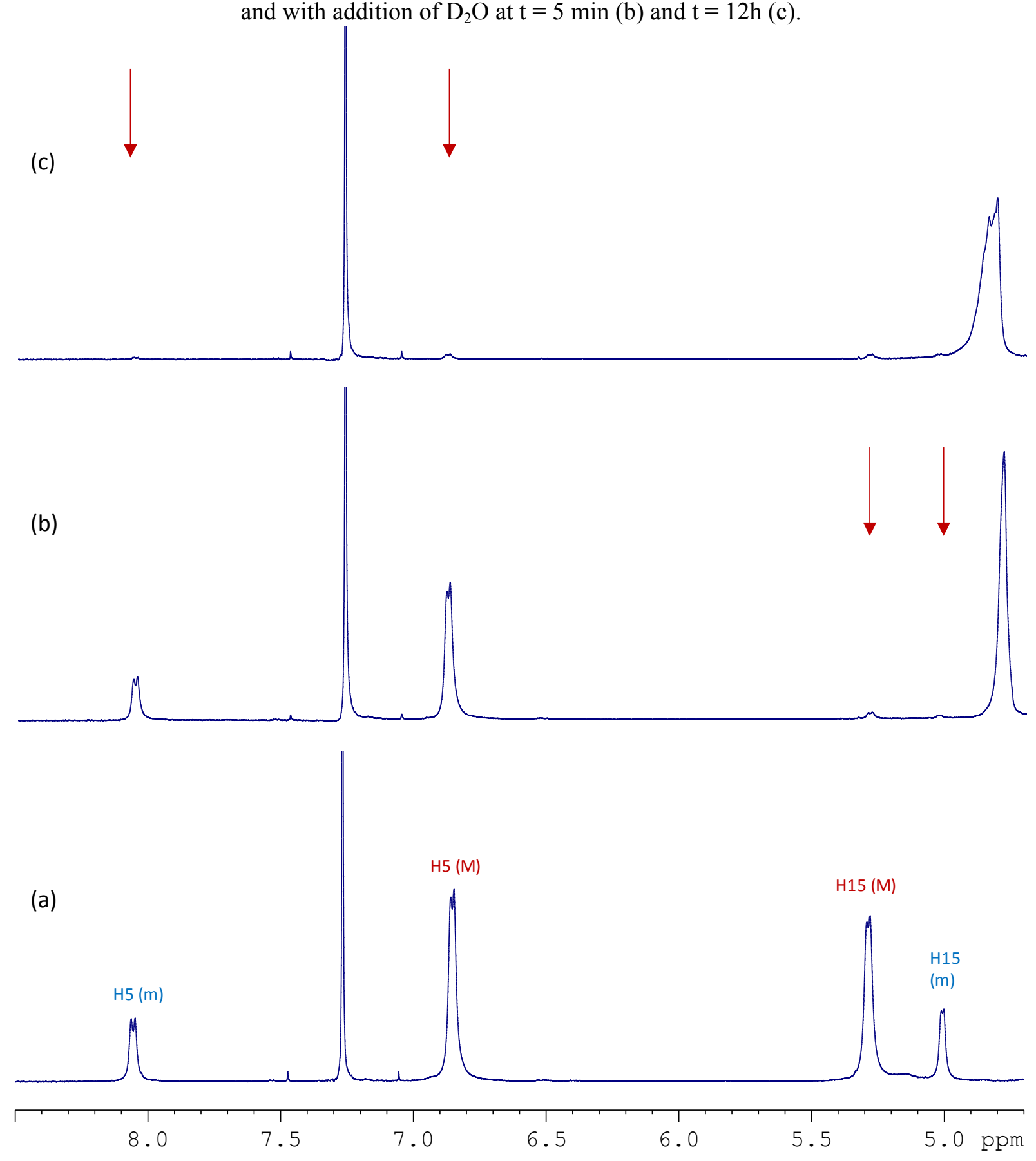


Table S1. ${ }^{1} \mathrm{H},{ }^{13} \mathrm{C}$ and ${ }^{19} \mathrm{~F}$ chemical shift of the main (trans) form of (-)-11 $\left(\mathrm{CDCl}_{3}, 500 \mathrm{MHz}, 298 \mathrm{~K}\right)$.

\begin{tabular}{|c|c|c|c|}
\hline $\mathrm{N}^{\circ} \mathrm{H} / \mathrm{C}$ & $\delta{ }^{1} \mathrm{H}(\mathrm{ppm})$ & $\delta^{19} \mathrm{~F}(\mathrm{ppm})$ & $\delta{ }^{13} \mathrm{C}(\mathrm{ppm})$ \\
\hline 1 & 3.76 & & 52.5 \\
\hline 2 & - & & 173.0 \\
\hline 3 & 4.55 & & 48.1 \\
\hline 4 & 1.39 & & 18.2 \\
\hline 5 & 6.86 & & - \\
\hline 6 & - & & 168.8 \\
\hline 7 & 4.45 & & 61.6 \\
\hline 8 & 2.23 & & 21.5 \\
\hline 9 & - & -200.9 & 81.5 \\
\hline $10 \mathrm{a}$ & 4.29 & & 49.5 \\
\hline $10 \mathrm{~b}$ & 4.00 & & 14.4 \\
\hline $11 \mathrm{a}$ & 1.59 & & 173.3 \\
\hline $11 \mathrm{~b}$ & 0.81 & & 48.1 \\
\hline 12 & - & & 18.2 \\
\hline 13 & 4.40 & & - \\
\hline 14 & 1.33 & & 155.1 \\
\hline 15 & 5.29 & & 79.9 \\
\hline 16 & - & & 28.3 \\
\hline 17 & - & & \\
\hline 18 & 1.43 & & \multicolumn{2}{|c|}{} \\
\hline
\end{tabular}


Table S2. ${ }^{1} \mathrm{H},{ }^{13} \mathrm{C}$ and ${ }^{19} \mathrm{~F}$ chemical shift of the minor (cis) form of (-)-11 $\left(\mathrm{CDCl}_{3}, 500 \mathrm{MHz}, 298 \mathrm{~K}\right)$.

\begin{tabular}{|c|c|c|c|}
\hline $\mathrm{N}^{\circ} \mathrm{H} / \mathrm{C}$ & $\delta^{1} \mathrm{H}(\mathrm{ppm})$ & $\delta^{19} \mathrm{~F}(\mathrm{ppm})$ & $\delta^{13} \mathrm{C}(\mathrm{ppm})$ \\
\hline 1 & 3.69 & & 52.2 \\
\hline 2 & - & & 172.7 \\
\hline 3 & 4.59 & & 48.5 \\
\hline 4 & 1.51 & & 16.6 \\
\hline 5 & 8.06 & & - \\
\hline 6 & - & & 169.2 \\
\hline 7 & 4.10 & & 63.0 \\
\hline 8 & 2.53 & & 23.5 \\
\hline 9 & - & -204.1 & 80.2 \\
\hline $10 a$ & 4.56 & & \multirow{2}{*}{47.9} \\
\hline $10 \mathrm{~b}$ & 3.32 & & \\
\hline $11 a$ & 1.59 & & \multirow{2}{*}{14.5} \\
\hline $11 b$ & 0.66 & & \\
\hline 12 & - & & 172.7 \\
\hline 13 & 4.17 & & 48.5 \\
\hline 14 & 1.22 & & 16.7 \\
\hline 15 & 5.01 & & - \\
\hline 16 & - & & 155.1 \\
\hline 17 & - & & 80.4 \\
\hline 18 & 1.39 & & 28.2 \\
\hline
\end{tabular}


Table S3: Experimental NOEs, ${ }^{3} J_{\mathrm{HH}}, \mathrm{H} / \mathrm{D}$ exchange and theoretical measured distances (d) for the compound (-)-11.

\begin{tabular}{|c|c|c|c|c|}
\hline & \multicolumn{2}{|c|}{ Trans conformer } & \multicolumn{2}{|c|}{ Cis conformer } \\
\hline & $\mathrm{NOE} /{ }^{3} J_{\mathrm{HH}}(\mathrm{Hz}) / \mathrm{d}(\AA)$ & H/D & $\mathrm{NOE} /{ }^{3} J_{\mathrm{HH}}(\mathrm{Hz}) / \mathrm{d}(\AA)$ & H/D \\
\hline NH5 - H13 & $\mathrm{W} /--/ 4,7$ & & $\mathrm{~S} /--/ 2,9$ & \\
\hline NH5 - H7 & $\mathrm{S} / \mathrm{--} / 2,4$ & & $\mathrm{M} /--/ 3,3$ & \\
\hline NH5 - H14 & $\mathrm{M} /--/ 3,7$ & NH5 & $\mathrm{W} /--/ 4,9$ & NH5 \\
\hline NH5 - H3 & $\mathrm{S} / 7,2 / 2,9$ & Slow & $\mathrm{S} / 7,9 / 2,8$ & \\
\hline NH5 - H4 & $\mathrm{S} /--/ 2,8$ & & $\mathrm{~S} /--/ 3,3$ & \\
\hline NH15 - H13 & $\mathrm{M} / 7,8 / 2,8$ & NH15 & $\mathrm{S} / 5,4 / 2,9$ & NH15 \\
\hline NH15 - H14 & $\mathrm{S} /--/ 3,0$ & Fast & $\mathrm{S} / \mathrm{--} / 2,8$ & Fast \\
\hline H13 - H10 & $\mathrm{S} /--/ 2,0$ & & $--/--/ 4,5$ & \\
\hline H14 - H10 & $\mathrm{S} / \mathrm{--} / 2,8$ & & $--/--/ 4,5$ & \\
\hline H14 - H10' & $\mathrm{S} /--/ 2,4$ & & $--/--/ 5,3$ & \\
\hline H7 - H13 & $--/--/ 4,4$ & & $M /--/ 2,2$ & \\
\hline
\end{tabular}

(--) not observed

Strong (S), medium (M) and weak (W) refer to a NOE effect arising from inter-proton distances of 1.5-2.5 $\AA, 2.5-3.5 \AA$ and over $3.5 \AA$ respectively. 
Table S4: Geometrical features of the optimized structure at B3LYP/cc-PVTZ level of theory for and the experimental ${ }^{3} J_{\mathrm{HN} 15-\mathrm{CH} 13}$ scalar coupling constants $(\mathrm{Hz})$ compound (-)-11.

\begin{tabular}{lcc}
\hline & Trans conformer & Cis conformer \\
\hline$\Phi(\mathrm{NH} 15-\mathrm{CH} 13)$ & -- & $-65^{\circ}$ \\
$\Psi(\mathrm{CH} 13-\mathrm{CO} 12)$ & -- & $139^{\circ}$ \\
$\Phi(\mathrm{N} 11-\mathrm{CH} 7)$ & $-86^{\circ}$ & $-91^{\circ}$ \\
$\Psi(\mathrm{CH} 7-\mathrm{CO6})$ & $57^{\circ}$ & $-6^{\circ}$ \\
\hline${ }^{3} J_{\mathrm{HN} 15-\mathrm{CH} 13}$ & 7.8 & 5.4 \\
\hline
\end{tabular}




\section{Crystallographic data}

\section{DATA COLLECTION}

The crystal structure of (-)-10 $\left[\mathrm{C}_{15} \mathrm{H}_{23} \mathrm{FN}_{2} \mathrm{O}_{5}\right]$ has been determined from single crystal X-Ray diffraction. The chosen crystal was stuck on a glass fibre and mounted on the full three-circle goniometer of a Bruker SMART APEX diffractometer with a CCD area detector. Three sets of exposures (a total of 1800 frames) were recorded, corresponding to three $\omega$ scans (steps of $0.3^{\circ}$ ), for three different values of $\phi$. The details of data collection are given in annexe 1.

The cell parameters and the orientation matrix of the crystal were preliminary determined by using SMART Software ${ }^{1}$. Data integration and global cell refinement were performed with SAINT Software ${ }^{2}$. Intensities were corrected for Lorentz, polarisation, decay and absorption effects (SAINT and SADABS Softwares) and reduced to $\mathrm{F}_{\mathrm{O}}{ }^{2}$. The program package Win $\mathrm{GX}^{3}$ was used for space group determination, structure solution and refinement.

\section{DATA REFINEMENT}

The standard space group $P 2_{1} 2_{1} 2_{1}\left(n^{\circ} 19\right)$ was determined from systematic extinctions and relative $F_{0}{ }^{2}$ of equivalent reflections. The structure was solved by direct methods ${ }^{4}$. Anisotropic displacement parameters were refined for all non-hydrogen atoms. Every Hydrogen atom were located from subsequent difference Fourier syntheses and placed with geometrical constraints (SHELXL ${ }^{5}$ ). The final cycle of full-matrix least-square refinement on $\mathrm{F}^{2}$ was based on 3690 observed reflections and 213 variable parameters and converged with unweighted and weighted agreement factors of:

$\mathrm{R} 1=0.0553, \mathrm{wR} 2=0.1275$ for 1593 reflections with $\mathrm{I}>2 \sigma \mathrm{l}$ and $\mathrm{R} 1=0.1659, \mathrm{wR} 2=0.1603$ for all data . 


\section{CRYSTALLOGRAPHIC DATA AND STRUCTURAL DESCRIPTION}

\section{Crystallographic data}

The crystal data are collected in Table 1. The full crystallographic parameters (atomic coordinates, bond length, angles and anisotropic displacements) are reported in annexe 2 .

Table S5: Crystal data of (-)-10.

\begin{tabular}{|c|c|}
\hline Chemical Formula & $\mathrm{C}_{15} \mathrm{H}_{23} \mathrm{FN}_{2} \mathrm{O}_{5}$ \\
\hline Molecular Weight / g. $\mathrm{mol}^{-1}$ & 330.3 \\
\hline Crystal System & Orthorhombic \\
\hline Space Group & $P 2_{1} 2_{1} 2_{1}$ \\
\hline $\mathrm{Z}, \mathrm{Z}^{\prime}$ (asymmetric units per unit cell) & 4,1 \\
\hline $\mathrm{a} / \AA$ & $6.5137(14)$ \\
\hline $\mathrm{b} / \AA$ & 15.684(3) \\
\hline $\mathrm{c} / \AA$ & $17.611(4)$ \\
\hline$\alpha /^{\circ}$ & 90 \\
\hline$\beta /^{\circ}$ & 90 \\
\hline$\gamma /{ }^{\circ}$ & 90 \\
\hline $\mathrm{V} / \AA^{3}$ & 1799.2(7) \\
\hline $\mathrm{d}_{\text {calc }} / \mathrm{g} \cdot \mathrm{cm}^{-3}$ & 1.220 \\
\hline $\mathrm{F}(000) / e^{-}$ & 704 \\
\hline Absorption coefficient $\mu\left(\mathrm{MoK}_{1}\right) / \mathrm{mm}^{-1}$ & 0.098 \\
\hline Absolute structure parameter & $2.2(18)$ \\
\hline
\end{tabular}

\section{Structural description}

The asymmetric unit is composed of one molecule of $\mathrm{C}_{15} \mathrm{H}_{23} \mathrm{FN}_{2} \mathrm{O}_{5}$ (Figures 1\&2). The absolute configuration of the stereogenic centre $\mathrm{C} 3$ is known (S), and thanks to this reference it is possible to determine the absolute configuration of three others centres, $C 6, C 7$ and $C 9$, that are respectively $S, R$ and $\mathrm{S}$.

Single crystals of $\mathrm{C}_{15} \mathrm{H}_{23} \mathrm{FN}_{2} \mathrm{O}_{5}(-)-10$ were grown from a dichloromethane/cyclohexane solution. 
Figure S77. Asymmetric unit in thermal ellipsoidal representation (ellipsoid contour 50\% probability level)

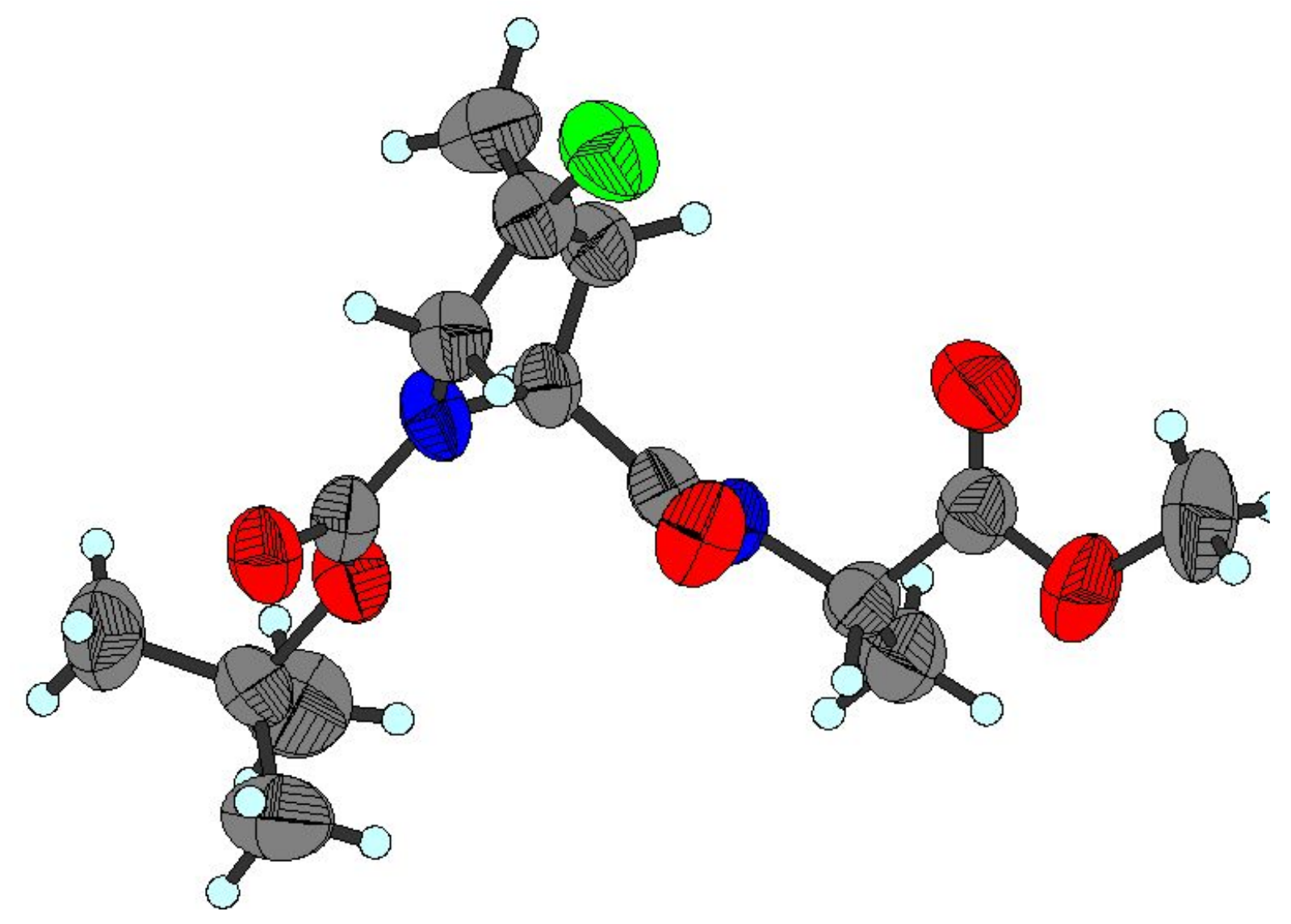

Figure S78. asymmetric unit with atom labels.

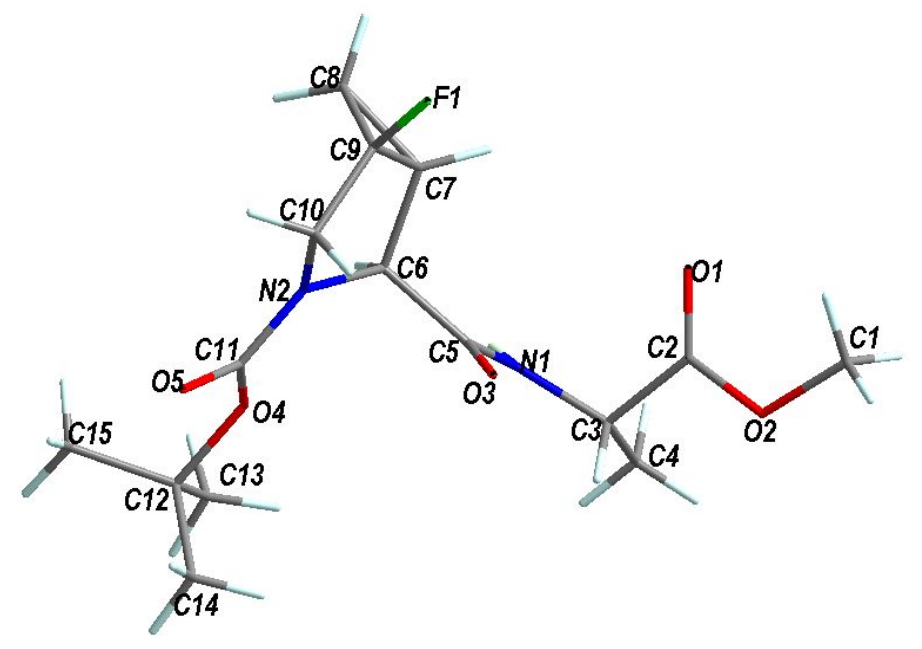


Figure S79. Molecular bond chain spreading along $a$ direction, Hydrogen bonds are displayed in dashed pink lines

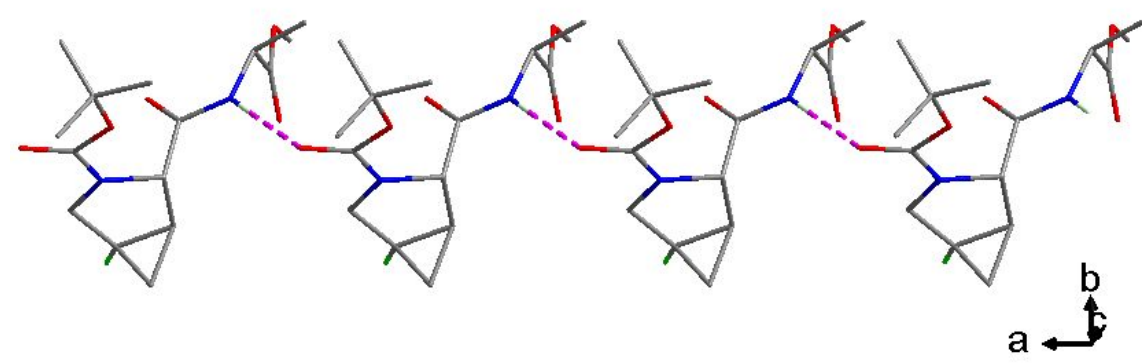

Table S6. Hydrogen bond table.

\begin{tabular}{|l|l|l|l|l|}
\hline$D-H \ldots A$ & $d(D-H)$ & $d(H \ldots A)$ & $d(D \ldots A)$ & $<(D H A)$ \\
\hline$N(1)-H(1) \ldots O(5) \# 1$ & 0.86 & 2.14 & $3.000(4)$ & 178.3 \\
\hline
\end{tabular}

Symmetry transformations used to generate equivalent atoms : $\# 1 x+1, y, z$

Figure S80. Projection along $a$ axis.

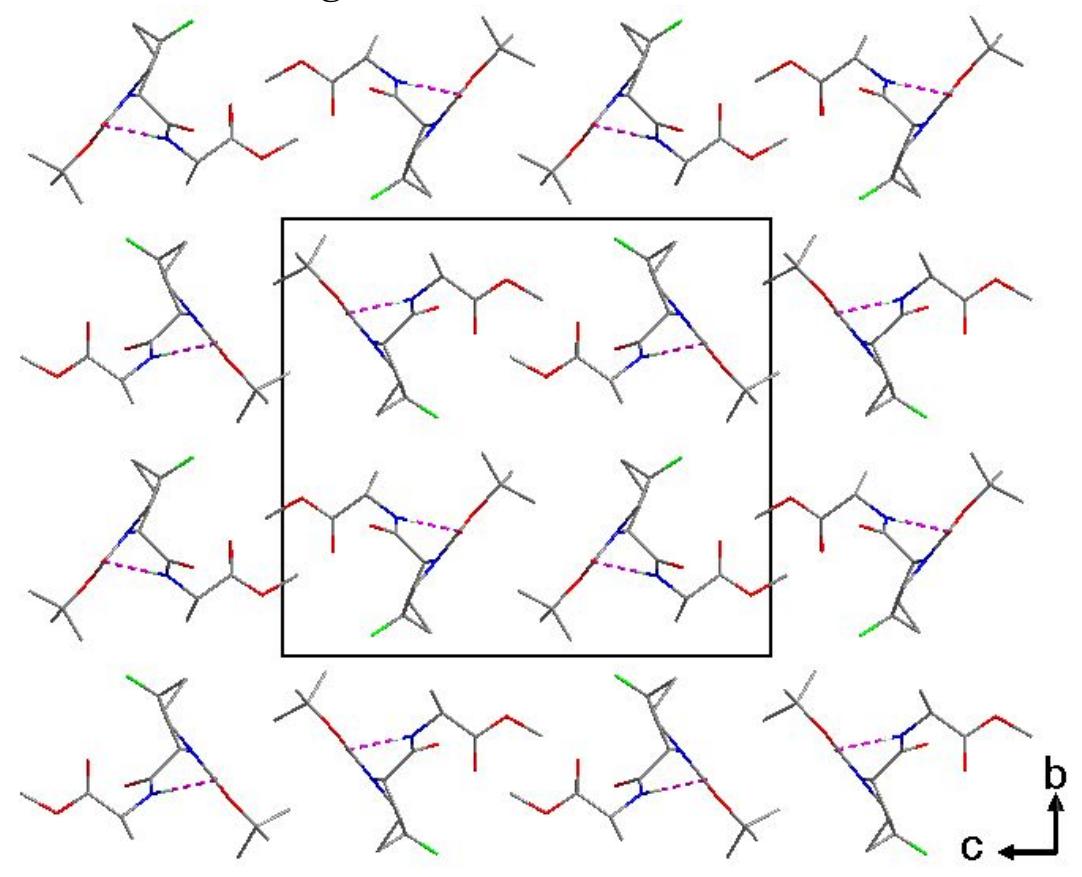


Figure S81. Projection along $c$ axis.

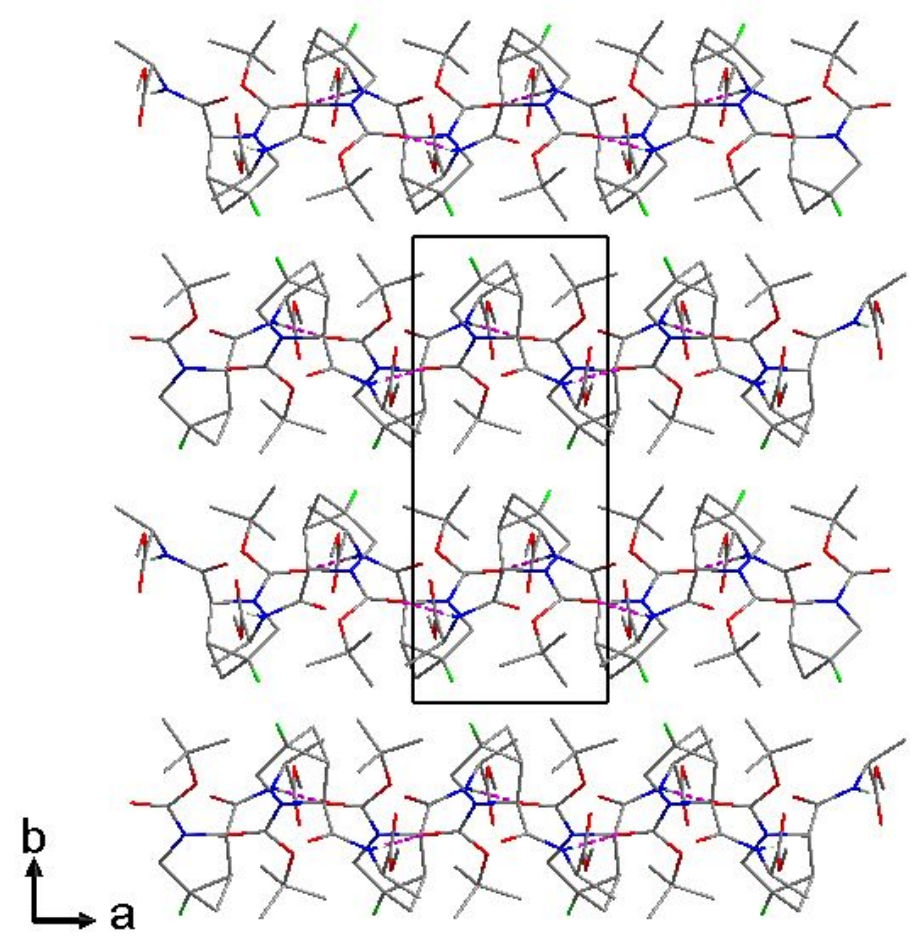

Figure S82. Projection along $b$ axis.

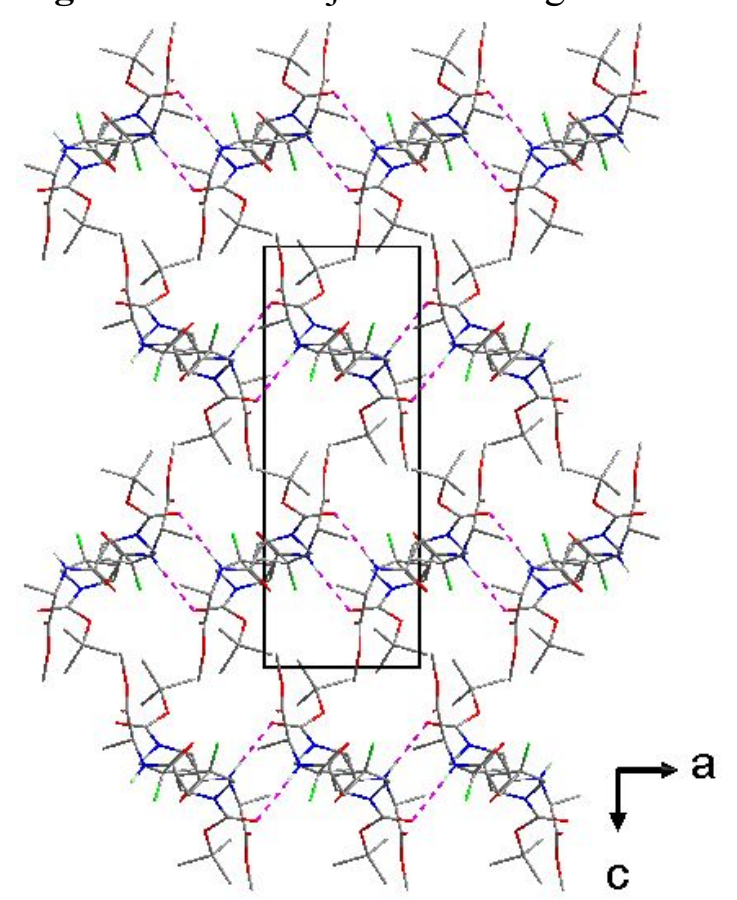

\section{Sofwares :}

(1)- SMART for WNT/2000 V5.622 (2001), Smart software reference manual, Bruker Advanced X Ray Solutions, Inc., Madison, Wisconsin, USA.

(2)- SAINT+ V6.02 (1999), Saint software reference manual, Bruker Advanced X Ray Solutions, Inc., Madison, Wisconsin, USA. 
(3)-WinGX: Version 1.70.01: An integrated system of Windows Programs for the solution, refinement and analysis of Single Crystal X-Ray Diffraction Data, By LouisJ. Farrugia, Dept. of chemistry, University of Glasgow.

L. J. Farrugia (1999) J. Appl. Cryst. 32, 837-838.

(4)-include in WinGX suite : SIR 92: A. Altomare, G. Cascarano, \& A. Gualardi (1993) J. Appl. Cryst. 26, 343-350; SHELXS-97: Sheldrick, G. M., (1990) Acta cryst, A46, 467.

(5)-include in WinGX suite: SHELXL-97 - a program for crystal structure refinement, G. M. .Sheldrick, University of Goettingen, Germany, 1997, release 97-2.

(6)-PowderCell for Windows (version 2.4) by Kraus W. \& Nolze G., Federal institute for materials Research and testing, Rudower Chausse 5, 12489 Berlin Germany. 
Table S7. DATA COLLECTION FOR (-)-10.

\begin{tabular}{|c|c|}
\hline Date & $14 / 11 / 17$ \\
\hline Temperature / $K$ & RT \\
\hline Radiation & $\operatorname{Mo}-K \alpha_{1}(\lambda=0.71073 \AA)$ \\
\hline Monochromator & Graphite \\
\hline Collimator $/ \mathrm{mm}$ & 0.5 \\
\hline Generator set & $50 \mathrm{kV} 40 \mathrm{~mA}$ \\
\hline Crystal-detector distance / $\mathrm{mm}$ & 60 \\
\hline Detector $2 \theta$ angle $/{ }^{\circ}$ & -28 \\
\hline$\omega$ oscillations $/{ }^{\circ}$ & -0.3 \\
\hline$\omega \operatorname{scan} 1$ & $\chi=54.7^{\circ}, \phi=0^{\circ},-28^{\circ} \leq \omega \leq-208^{\circ}$ \\
\hline$\omega \operatorname{scan} 2$ & $\chi=54.7^{\circ}, \phi=120^{\circ},-28^{\circ} \leq \omega \leq-208^{\circ}$ \\
\hline$\omega \operatorname{scan} 3$ & $\chi=54.7^{\circ}, \phi=240^{\circ},-28^{\circ} \leq \omega \leq-208^{\circ}$ \\
\hline Time exposure / $s$ & 20 \\
\hline Total number of reflections & 14303 \\
\hline Unique reflections $\left[F_{o}>4.0 \sigma\left(F_{o}\right)\right]$ & $3690 / 1593$ \\
\hline$\theta$ range $/^{\circ}$ & 1.74 to 26.46 \\
\hline hkl range & $-8 \leq h \leq 8,-19 \leq k \leq 19,-21 \leq 1 \leq 22$ \\
\hline $\mathrm{R}_{\text {int }}=\Sigma\left[\mid \mathrm{F}_{\mathrm{O}}^{2}-\mathrm{F}_{\mathrm{O}}^{2}(\right.$ mean $\left.) \mid\right] / \Sigma\left[\mathrm{F}_{\mathrm{O}}{ }^{2}\right]$ & 0.0879 \\
\hline Completeness to $\theta=26.40 / \%$ & 99.5 \\
\hline
\end{tabular}

Table S8. REFINEMENT DATA FOR (-)-10.

\begin{tabular}{|l|l|}
\hline Number of reflections $(\mathrm{n})\left(\right.$ with $\left.\mathrm{F}_{\mathrm{O}}>4.0 \sigma\left(\mathrm{F}_{\mathrm{O}}\right)\right)$ & 1593 \\
\hline Number of refined parameters $(\mathrm{p}) /$ restraints & $213 / 0$ \\
\hline Final $\mathrm{R}$ indices [l>2sigma(I)] & $\mathrm{R} 1=0.0553, \mathrm{WR2}=0.1275$ \\
\hline R indices (all data) & $\mathrm{R} 1=0.1659, \mathrm{wR2}=0.1603$ \\
\hline Goodness of Fit indicator (Restrained GooF) & 0.869 \\
\hline Maximum peak in Final Difference Map $/ e^{-\AA^{-3}}$ & 0.408 \\
\hline Maximum hole in Final Difference Map $/ e^{-} \AA^{-3}$ & -0.107 \\
\hline
\end{tabular}


$\mathrm{R}_{1}=\Sigma\left(|| \mathrm{F}_{\mathrm{O}}|-| \mathrm{F}_{\mathrm{C}} \mid\right) / \Sigma\left|\mathrm{F}_{\mathrm{O}}\right|$

$w R_{2}=\left[\Sigma\left[w\left(F_{O}^{2}-F_{c}^{2}\right)^{2}\right] / \Sigma\left[w\left(F_{O}^{2}\right)^{2}\right]\right]^{1 / 2}$

GooF $=\left[\Sigma\left[w\left(F_{o}^{2}-F_{c}^{2}\right)^{2}\right] /(n-p)\right]^{1 / 2}$ 
ANNEXE 2 : CRYSTALLOGRAPHIC DATA

Table S9. Atomic coordinates $\left(\times 10^{4}\right)$ and equivalent isotropic displacement parameters $\left(\AA^{2} \mathrm{x}\right.$ $\left.10^{3}\right)$.

$\mathrm{U}(\mathrm{eq})$ is defined as one third of the trace of the orthogonalized Uij tensor

\begin{tabular}{|c|c|c|c|c|}
\hline & $x$ & $y$ & $z$ & $\mathrm{U}(\mathrm{eq})$ \\
\hline$C(1)$ & 14211(9) & $8164(4)$ & 4692(2) & $129(2)$ \\
\hline$C(2)$ & $13835(6)$ & $8142(3)$ & $6034(2)$ & $68(1)$ \\
\hline$C(3)$ & $13430(7)$ & $8694(2)$ & $6722(2)$ & $61(1)$ \\
\hline$C(4)$ & $15327(7)$ & 9194(3) & 6940(2) & $81(1)$ \\
\hline$C(5)$ & $10846(6)$ & 7815(3) & $7320(2)$ & $58(1)$ \\
\hline$C(6)$ & $10380(6)$ & $7147(2)$ & $7921(2)$ & $54(1)$ \\
\hline$C(7)$ & 10599(6) & $6278(3)$ & $7577(2)$ & $65(1)$ \\
\hline$C(8)$ & 9853(7) & $5527(3)$ & 8040(3) & $85(1)$ \\
\hline$C(9)$ & $8505(6)$ & $5927(3)$ & $7477(2)$ & $66(1)$ \\
\hline$C(10)$ & $6960(6)$ & $6567(3)$ & $7709(2)$ & $66(1)$ \\
\hline$C(11)$ & 7361(7) & 7765(3) & $8575(2)$ & $61(1)$ \\
\hline$C(12)$ & 8338(8) & $8917(3)$ & $9475(2)$ & 73(1) \\
\hline$C(13)$ & 10461(8) & 9202(3) & 9705(3) & $103(2)$ \\
\hline$C(14)$ & 7145(9) & 9624(3) & 9106(3) & $113(2)$ \\
\hline$C(15)$ & 7197(9) & $8526(3)$ & $10142(2)$ & $104(2)$ \\
\hline$F(1)$ & 7984(4) & $5468(2)$ & $6838(1)$ & $95(1)$ \\
\hline$N(1)$ & $12709(5)$ & $8164(2)$ & $7343(2)$ & $58(1)$ \\
\hline$N(2)$ & 8209(5) & $7169(2)$ & $8143(2)$ & $63(1)$ \\
\hline $\mathrm{O}(1)$ & $14116(5)$ & $7389(2)$ & $6043(2)$ & 91(1) \\
\hline $\mathrm{O}(2)$ & $13852(5)$ & $8609(2)$ & $5407(2)$ & 102(1) \\
\hline $\mathrm{O}(3)$ & 9605(4) & $7977(2)$ & $6818(2)$ & $80(1)$ \\
\hline $\mathrm{O}(4)$ & $8807(4)$ & $8249(2)$ & $8906(1)$ & $69(1)$ \\
\hline $\mathrm{O}(5)$ & $5502(5)$ & $7843(2)$ & $8667(2)$ & $72(1)$ \\
\hline
\end{tabular}


Table S10. Hydrogen coordinates $\left(\times 10^{4}\right)$ and equivalent isotropic displacement parameters $\left(\AA^{2} \times 10^{3}\right)$.

$U(e q)$ is defined as one third of the trace of the orthogonalized Uij tensor

\begin{tabular}{|l|l|l|l|l|}
\hline & $x$ & $y$ & $z$ & $U(e q)$ \\
\hline$H(1 A)$ & 13860 & 7573 & 4749 & 194 \\
\hline$H(1 B)$ & 15632 & 8213 & 4554 & 194 \\
\hline$H(1 C)$ & 13376 & 8413 & 4301 & 194 \\
\hline$H(3)$ & 12342 & 9100 & 6593 & 74 \\
\hline$H(4 A)$ & 15029 & 9544 & 7373 & 121 \\
\hline$H(4 B)$ & 15738 & 9548 & 6523 & 121 \\
\hline$H(4 C)$ & 16418 & 8806 & 7063 & 121 \\
\hline$H(6)$ & 11278 & 7214 & 8364 & 65 \\
\hline$H(7)$ & 11627 & 6185 & 7179 & 78 \\
\hline$H(8 A)$ & 10413 & 4969 & 7923 & 102 \\
\hline$H(8 B)$ & 9551 & 5622 & 8573 & 102 \\
\hline$H(10 A)$ & 6335 & 6839 & 7271 & 79 \\
\hline$H(10 B)$ & 5895 & 6314 & 8021 & 79 \\
\hline$H(13 A)$ & 11135 & 8750 & 9975 & 154 \\
\hline$H(13 B)$ & 10360 & 9695 & 10026 & 154 \\
\hline$H(13 C)$ & 11240 & 9343 & 9259 & 154 \\
\hline$H(14 A)$ & 7869 & 9821 & 8665 & 169 \\
\hline$H(14 B)$ & 6986 & 10087 & 9459 & 169 \\
\hline$H(14 C)$ & 5816 & 9417 & 8959 & 169 \\
\hline$H(15 A)$ & 5842 & 8364 & 9985 & 155 \\
\hline$H(15 B)$ & 7104 & 8936 & 10546 & 155 \\
\hline$H(15 C)$ & 7926 & 8032 & 10318 & 155 \\
\hline$H(1)$ & 13489 & 8076 & 7729 & 69 \\
\hline & & & & \\
\hline & & & & \\
\hline & & & & \\
\hline & & & & \\
\hline & & & & \\
\hline
\end{tabular}


Table S11. Bond lengths $(\AA)$.

\begin{tabular}{|ll|ll|}
\hline $\mathrm{C}(1)-\mathrm{O}(2)$ & $1.460(5)$ & $\mathrm{C}(8)-\mathrm{H}(8 \mathrm{~B})$ & 0.97 \\
\hline $\mathrm{C}(1)-\mathrm{H}(1 \mathrm{~A})$ & 0.96 & $\mathrm{C}(9)-\mathrm{F}(1)$ & $1.378(4)$ \\
\hline $\mathrm{C}(1)-\mathrm{H}(1 \mathrm{~B})$ & 0.96 & $\mathrm{C}(9)-\mathrm{C}(10)$ & $1.479(5)$ \\
\hline $\mathrm{C}(1)-\mathrm{H}(1 \mathrm{C})$ & 0.96 & $\mathrm{C}(10)-\mathrm{N}(2)$ & $1.463(5)$ \\
\hline $\mathrm{C}(2)-\mathrm{O}(1)$ & $1.195(5)$ & $\mathrm{C}(10)-\mathrm{H}(10 \mathrm{~A})$ & 0.97 \\
\hline $\mathrm{C}(2)-\mathrm{O}(2)$ & $1.325(5)$ & $\mathrm{C}(10)-\mathrm{H}(10 \mathrm{~B})$ & 0.97 \\
\hline $\mathrm{C}(2)-\mathrm{C}(3)$ & $1.512(6)$ & $\mathrm{C}(11)-\mathrm{O}(5)$ & $1.228(5)$ \\
\hline $\mathrm{C}(3)-\mathrm{N}(1)$ & $1.452(4)$ & $\mathrm{C}(11)-\mathrm{N}(2)$ & $1.327(5)$ \\
\hline $\mathrm{C}(3)-\mathrm{C}(4)$ & $1.513(6)$ & $\mathrm{C}(11)-\mathrm{O}(4)$ & $1.342(5)$ \\
\hline $\mathrm{C}(3)-\mathrm{H}(3)$ & 0.98 & $\mathrm{C}(12)-\mathrm{O}(4)$ & $1.482(5)$ \\
\hline $\mathrm{C}(4)-\mathrm{H}(4 \mathrm{~A})$ & 0.96 & $\mathrm{C}(12)-\mathrm{C}(14)$ & $1.502(6)$ \\
\hline $\mathrm{C}(4)-\mathrm{H}(4 \mathrm{~B})$ & 0.96 & $\mathrm{C}(12)-\mathrm{C}(13)$ & $1.509(7)$ \\
\hline $\mathrm{C}(4)-\mathrm{H}(4 \mathrm{C})$ & 0.96 & $\mathrm{C}(12)-\mathrm{C}(15)$ & $1.520(6)$ \\
\hline $\mathrm{C}(5)-\mathrm{O}(3)$ & $1.225(4)$ & $\mathrm{C}(13)-\mathrm{H}(13 \mathrm{~A})$ & 0.96 \\
\hline $\mathrm{C}(5)-\mathrm{N}(1)$ & $1.332(5)$ & $\mathrm{C}(13)-\mathrm{H}(13 \mathrm{~B})$ & 0.96 \\
\hline $\mathrm{C}(5)-\mathrm{C}(6)$ & $1.520(5)$ & $\mathrm{C}(13)-\mathrm{H}(13 \mathrm{C})$ & 0.96 \\
\hline $\mathrm{C}(6)-\mathrm{N}(2)$ & $1.467(5)$ & $\mathrm{C}(14)-\mathrm{H}(14 \mathrm{~A})$ & 0.96 \\
\hline $\mathrm{C}(6)-\mathrm{C}(7)$ & $1.499(5)$ & $\mathrm{C}(14)-\mathrm{H}(14 \mathrm{~B})$ & 0.96 \\
\hline $\mathrm{C}(6)-\mathrm{H}(6)$ & 0.98 & $\mathrm{C}(14)-\mathrm{H}(14 \mathrm{C})$ & 0.96 \\
\hline $\mathrm{C}(7)-\mathrm{C}(9)$ & $1.481(6)$ & $\mathrm{C}(15)-\mathrm{H}(15 \mathrm{~A})$ & 0.96 \\
\hline $\mathrm{C}(7)-\mathrm{C}(8)$ & $1.513(6)$ & $\mathrm{C}(15)-\mathrm{H}(15 \mathrm{~B})$ & 0.96 \\
\hline $\mathrm{C}(7)-\mathrm{H}(7)$ & 0.98 & $\mathrm{C}(15)-\mathrm{H}(15 \mathrm{C})$ & 0.96 \\
\hline $\mathrm{C}(8)-\mathrm{C}(9)$ & $1.466(6)$ & $\mathrm{N}(1)-\mathrm{H}(1)$ & 0.86 \\
\hline $\mathrm{C}(8)-\mathrm{H}(8 \mathrm{~A})$ & 0.97 & & \\
\hline & & & \\
\hline
\end{tabular}


Table S12. Angles $\left(^{\circ}\right)$.

\begin{tabular}{|lllll|}
\hline $\mathrm{O}(2)-\mathrm{C}(1)-\mathrm{H}(1 \mathrm{~A})$ & 109.5 & $\mathrm{~F}(1)-\mathrm{C}(9)-\mathrm{C}(7)$ & $121.2(4)$ \\
\hline $\mathrm{O}(2)-\mathrm{C}(1)-\mathrm{H}(1 \mathrm{~B})$ & 109.5 & $\mathrm{C}(8)-\mathrm{C}(9)-\mathrm{C}(7)$ & $61.8(3)$ \\
\hline $\mathrm{H}(1 \mathrm{~A})-\mathrm{C}(1)-\mathrm{H}(1 \mathrm{~B})$ & 109.5 & $\mathrm{C}(10)-\mathrm{C}(9)-\mathrm{C}(7)$ & $110.0(3)$ \\
\hline $\mathrm{O}(2)-\mathrm{C}(1)-\mathrm{H}(1 \mathrm{C})$ & 109.5 & $\mathrm{~N}(2)-\mathrm{C}(10)-\mathrm{C}(9)$ & $101.8(3)$ \\
\hline $\mathrm{H}(1 \mathrm{~A})-\mathrm{C}(1)-\mathrm{H}(1 \mathrm{C})$ & 109.5 & $\mathrm{~N}(2)-\mathrm{C}(10)-\mathrm{H}(10 \mathrm{~A})$ & 111.4 \\
\hline $\mathrm{H}(1 \mathrm{~B})-\mathrm{C}(1)-\mathrm{H}(1 \mathrm{C})$ & 109.5 & $\mathrm{C}(9)-\mathrm{C}(10)-\mathrm{H}(10 \mathrm{~A})$ & 111.4 \\
\hline $\mathrm{O}(1)-\mathrm{C}(2)-\mathrm{O}(2)$ & $123.8(4)$ & $\mathrm{N}(2)-\mathrm{C}(10)-\mathrm{H}(10 \mathrm{~B})$ & 111.4 \\
\hline $\mathrm{O}(1)-\mathrm{C}(2)-\mathrm{C}(3)$ & $125.6(4)$ & $\mathrm{C}(9)-\mathrm{C}(10)-\mathrm{H}(10 \mathrm{~B})$ & 111.4 \\
\hline $\mathrm{O}(2)-\mathrm{C}(2)-\mathrm{C}(3)$ & $110.6(4)$ & $\mathrm{H}(10 \mathrm{~A})-\mathrm{C}(10)-\mathrm{H}(10 \mathrm{~B})$ & 109.3 \\
\hline $\mathrm{N}(1)-\mathrm{C}(3)-\mathrm{C}(2)$ & $109.4(3)$ & $\mathrm{O}(5)-\mathrm{C}(11)-\mathrm{N}(2)$ & $123.8(4)$ \\
\hline $\mathrm{N}(1)-\mathrm{C}(3)-\mathrm{C}(4)$ & $111.7(3)$ & $\mathrm{O}(5)-\mathrm{C}(11)-\mathrm{O}(4)$ & $125.3(4)$ \\
\hline $\mathrm{C}(2)-\mathrm{C}(3)-\mathrm{C}(4)$ & $111.0(3)$ & $\mathrm{N}(2)-\mathrm{C}(11)-\mathrm{O}(4)$ & $110.8(4)$ \\
\hline $\mathrm{N}(1)-\mathrm{C}(3)-\mathrm{H}(3)$ & 108.2 & $\mathrm{O}(4)-\mathrm{C}(12)-\mathrm{C}(14)$ & $109.7(3)$ \\
\hline $\mathrm{C}(2)-\mathrm{C}(3)-\mathrm{H}(3)$ & 108.2 & $\mathrm{O}(4)-\mathrm{C}(12)-\mathrm{C}(13)$ & $101.6(4)$ \\
\hline $\mathrm{C}(4)-\mathrm{C}(3)-\mathrm{H}(3)$ & 108.2 & $\mathrm{C}(14)-\mathrm{C}(12)-\mathrm{C}(13)$ & $111.8(4)$ \\
\hline $\mathrm{C}(3)-\mathrm{C}(4)-\mathrm{H}(4 \mathrm{~A})$ & 109.5 & $\mathrm{O}(4)-\mathrm{C}(12)-\mathrm{C}(15)$ & $109.7(3)$ \\
\hline $\mathrm{C}(3)-\mathrm{C}(4)-\mathrm{H}(4 \mathrm{~B})$ & 109.5 & $\mathrm{C}(14)-\mathrm{C}(12)-\mathrm{C}(15)$ & $112.3(5)$ \\
\hline $\mathrm{H}(4 \mathrm{~A})-\mathrm{C}(4)-\mathrm{H}(4 \mathrm{~B})$ & 109.5 & $\mathrm{C}(13)-\mathrm{C}(12)-\mathrm{C}(15)$ & $111.1(4)$ \\
\hline $\mathrm{C}(3)-\mathrm{C}(4)-\mathrm{H}(4 \mathrm{C})$ & 109.5 & $\mathrm{C}(12)-\mathrm{C}(13)-\mathrm{H}(13 \mathrm{~A})$ & 109.5 \\
\hline $\mathrm{H}(4 \mathrm{~A})-\mathrm{C}(4)-\mathrm{H}(4 \mathrm{C})$ & 109.5 & $\mathrm{C}(12)-\mathrm{C}(13)-\mathrm{H}(13 \mathrm{~B})$ & 109.5 \\
\hline $\mathrm{H}(4 \mathrm{~B})-\mathrm{C}(4)-\mathrm{H}(4 \mathrm{C})$ & 109.5 & $\mathrm{H}(13 \mathrm{~A})-\mathrm{C}(13)-\mathrm{H}(13 \mathrm{~B})$ & 109.5 \\
\hline $\mathrm{O}(3)-\mathrm{C}(5)-\mathrm{N}(1)$ & $122.5(4)$ & $\mathrm{C}(12)-\mathrm{C}(13)-\mathrm{H}(13 \mathrm{C})$ & 109.5 \\
\hline $\mathrm{O}(3)-\mathrm{C}(5)-\mathrm{C}(6)$ & $121.0(4)$ & $\mathrm{H}(13 \mathrm{~A})-\mathrm{C}(13)-\mathrm{H}(13 \mathrm{C})$ & 109.5 \\
\hline $\mathrm{N}(1)-\mathrm{C}(5)-\mathrm{C}(6)$ & $116.3(4)$ & $\mathrm{H}(13 \mathrm{~B})-\mathrm{C}(13)-\mathrm{H}(13 \mathrm{C})$ & 109.5 \\
\hline $\mathrm{N}(2)-\mathrm{C}(6)-\mathrm{C}(7)$ & $102.7(3)$ & $\mathrm{C}(12)-\mathrm{C}(14)-\mathrm{H}(14 \mathrm{~A})$ & 109.5 \\
\hline $\mathrm{N}(2)-\mathrm{C}(6)-\mathrm{C}(5)$ & $111.2(3)$ & $\mathrm{C}(12)-\mathrm{C}(14)-\mathrm{H}(14 \mathrm{~B})$ & 109.5 \\
\hline $\mathrm{C}(7)-\mathrm{C}(6)-\mathrm{C}(5)$ & $109.0(3)$ & $\mathrm{H}(14 \mathrm{~A})-\mathrm{C}(14)-\mathrm{H}(14 \mathrm{~B})$ & 109.5 \\
\hline
\end{tabular}




\begin{tabular}{|ll|ll|}
\hline $\mathrm{C}(9)-\mathrm{C}(7)-\mathrm{C}(6)$ & $107.4(3)$ & $\mathrm{C}(12)-\mathrm{C}(15)-\mathrm{H}(15 \mathrm{~A})$ & 109.5 \\
\hline $\mathrm{C}(9)-\mathrm{C}(7)-\mathrm{C}(8)$ & $58.6(3)$ & $\mathrm{C}(12)-\mathrm{C}(15)-\mathrm{H}(15 \mathrm{~B})$ & 109.5 \\
\hline $\mathrm{C}(6)-\mathrm{C}(7)-\mathrm{C}(8)$ & $117.3(3)$ & $\mathrm{H}(15 \mathrm{~A})-\mathrm{C}(15)-\mathrm{H}(15 \mathrm{~B})$ & 109.5 \\
\hline $\mathrm{C}(9)-\mathrm{C}(7)-\mathrm{H}(7)$ & 119.3 & $\mathrm{C}(12)-\mathrm{C}(15)-\mathrm{H}(15 \mathrm{C})$ & 109.5 \\
\hline $\mathrm{C}(6)-\mathrm{C}(7)-\mathrm{H}(7)$ & 119.3 & $\mathrm{H}(15 \mathrm{~A})-\mathrm{C}(15)-\mathrm{H}(15 \mathrm{C})$ & 109.5 \\
\hline $\mathrm{C}(8)-\mathrm{C}(7)-\mathrm{H}(7)$ & 119.3 & $\mathrm{H}(15 \mathrm{~B})-\mathrm{C}(15)-\mathrm{H}(15 \mathrm{C})$ & 109.5 \\
\hline $\mathrm{C}(9)-\mathrm{C}(8)-\mathrm{C}(7)$ & $59.6(3)$ & $\mathrm{C}(5)-\mathrm{N}(1)-\mathrm{C}(3)$ & $120.4(3)$ \\
\hline $\mathrm{C}(9)-\mathrm{C}(8)-\mathrm{H}(8 \mathrm{~A})$ & 117.8 & $\mathrm{C}(5)-\mathrm{N}(1)-\mathrm{H}(1)$ & 119.8 \\
\hline $\mathrm{C}(7)-\mathrm{C}(8)-\mathrm{H}(8 \mathrm{~A})$ & 117.8 & $\mathrm{C}(3)-\mathrm{N}(1)-\mathrm{H}(1)$ & 119.8 \\
\hline $\mathrm{C}(9)-\mathrm{C}(8)-\mathrm{H}(8 \mathrm{~B})$ & 117.8 & $\mathrm{C}(11)-\mathrm{N}(2)-\mathrm{C}(10)$ & $121.6(4)$ \\
\hline $\mathrm{C}(7)-\mathrm{C}(8)-\mathrm{H}(8 \mathrm{~B})$ & 117.8 & $\mathrm{C}(11)-\mathrm{N}(2)-\mathrm{C}(6)$ & $124.8(3)$ \\
\hline$H(8 \mathrm{~A})-\mathrm{C}(8)-\mathrm{H}(8 \mathrm{~B})$ & 114.9 & $\mathrm{C}(10)-\mathrm{N}(2)-\mathrm{C}(6)$ & $112.5(3)$ \\
\hline $\mathrm{F}(1)-\mathrm{C}(9)-\mathrm{C}(8)$ & $118.4(4)$ & $\mathrm{C}(2)-\mathrm{O}(2)-\mathrm{C}(1)$ & $117.1(4)$ \\
\hline $\mathrm{F}(1)-\mathrm{C}(9)-\mathrm{C}(10)$ & $114.3(3)$ & $\mathrm{C}(11)-\mathrm{O}(4)-\mathrm{C}(12)$ & $123.3(3)$ \\
\hline $\mathrm{C}(8)-\mathrm{C}(9)-\mathrm{C}(10)$ & $120.8(4)$ & & \\
\hline
\end{tabular}


Table S13. Anisotropic displacement parameters $\left(\AA^{2} \times 10^{3}\right)$ The anisotropic displacement factor exponent takes the form: $-2 \pi^{2}\left[\mathrm{~h}^{2} \mathrm{a}^{* 2} \mathrm{U} 11+\ldots+2 \mathrm{~h} \mathrm{k} \mathrm{a}^{*} \mathrm{~b}^{*} \mathrm{U} 12\right]$

\begin{tabular}{|l|l|l|l|l|l|l|}
\hline & $\mathrm{U} 11$ & $\mathrm{U} 22$ & $\mathrm{U} 33$ & $\mathrm{U} 23$ & $\mathrm{U} 13$ & $\mathrm{U} 12$ \\
\hline $\mathrm{C}(1)$ & $161(6)$ & $174(5)$ & $53(3)$ & $6(3)$ & $29(3)$ & $-5(5)$ \\
\hline $\mathrm{C}(2)$ & $55(3)$ & $78(3)$ & $72(3)$ & $7(3)$ & $5(2)$ & $-2(2)$ \\
\hline $\mathrm{C}(3)$ & $59(3)$ & $60(2)$ & $65(3)$ & $11(2)$ & $7(2)$ & $4(2)$ \\
\hline $\mathrm{C}(4)$ & $70(3)$ & $83(3)$ & $89(3)$ & $2(2)$ & $10(3)$ & $-11(3)$ \\
\hline $\mathrm{C}(5)$ & $49(3)$ & $72(3)$ & $54(2)$ & $-10(2)$ & $1(2)$ & $3(2)$ \\
\hline $\mathrm{C}(6)$ & $46(2)$ & $68(3)$ & $49(2)$ & $-2(2)$ & $1(2)$ & $0(2)$ \\
\hline $\mathrm{C}(7)$ & $50(3)$ & $72(3)$ & $72(3)$ & $0(2)$ & $8(2)$ & $7(2)$ \\
\hline $\mathrm{C}(8)$ & $76(3)$ & $70(3)$ & $109(4)$ & $15(3)$ & $-5(3)$ & $-1(3)$ \\
\hline $\mathrm{C}(9)$ & $62(3)$ & $74(3)$ & $64(3)$ & $-10(2)$ & $-3(2)$ & $-4(2)$ \\
\hline $\mathrm{C}(10)$ & $57(3)$ & $76(3)$ & $65(2)$ & $-5(2)$ & $-1(2)$ & $-13(2)$ \\
\hline $\mathrm{C}(11)$ & $54(3)$ & $76(3)$ & $52(2)$ & $8(2)$ & $7(2)$ & $-1(3)$ \\
\hline $\mathrm{C}(12)$ & $97(4)$ & $65(3)$ & $57(3)$ & $-12(2)$ & $13(3)$ & $5(3)$ \\
\hline $\mathrm{C}(13)$ & $115(4)$ & $101(4)$ & $92(3)$ & $-17(3)$ & $-15(3)$ & $-26(3)$ \\
\hline $\mathrm{C}(14)$ & $142(5)$ & $74(3)$ & $121(4)$ & $-4(3)$ & $6(4)$ & $15(4)$ \\
\hline $\mathrm{C}(15)$ & $131(4)$ & $116(4)$ & $64(3)$ & $-6(3)$ & $23(3)$ & $-13(4)$ \\
\hline $\mathrm{F}(1)$ & $102(2)$ & $93(2)$ & $90(2)$ & $-34(1)$ & $-2(2)$ & $-10(2)$ \\
\hline $\mathrm{N}(1)$ & $45(2)$ & $74(2)$ & $55(2)$ & $7(2)$ & $0(2)$ & $-5(2)$ \\
\hline $\mathrm{N}(2)$ & $47(2)$ & $80(2)$ & $60(2)$ & $-15(2)$ & $10(2)$ & $-4(2)$ \\
\hline $\mathrm{O}(1)$ & $108(3)$ & $88(2)$ & $76(2)$ & $-7(2)$ & $11(2)$ & $20(2)$ \\
\hline $\mathrm{O}(2)$ & $135(3)$ & $111(2)$ & $60(2)$ & $23(2)$ & $15(2)$ & $-4(2)$ \\
\hline $\mathrm{O}(3)$ & $58(2)$ & $112(2)$ & $72(2)$ & $20(2)$ & $-15(2)$ & $-8(2)$ \\
\hline $\mathrm{O}(4)$ & $63(2)$ & $82(2)$ & $64(2)$ & $-13(2)$ & $6(2)$ & $-9(2)$ \\
\hline $\mathrm{O}(5)$ & $53(2)$ & $97(2)$ & $68(2)$ & $0(2)$ & $10(2)$ & $4(2)$ \\
\hline
\end{tabular}


Table S14. Torsion angles $\left(^{\circ}\right)$.

\begin{tabular}{|c|c|c|c|}
\hline $\mathrm{O}(1)-\mathrm{C}(2)-\mathrm{C}(3)-\mathrm{N}(1)$ & $-20.3(6)$ & $C(7)-C(9)-C(10)-N(2)$ & $-16.1(4)$ \\
\hline $\mathrm{O}(2)-\mathrm{C}(2)-\mathrm{C}(3)-\mathrm{N}(1)$ & $160.1(4)$ & $\mathrm{O}(3)-\mathrm{C}(5)-\mathrm{N}(1)-\mathrm{C}(3)$ & $-6.5(5)$ \\
\hline $\mathrm{O}(1)-\mathrm{C}(2)-\mathrm{C}(3)-\mathrm{C}(4)$ & $103.4(5)$ & $C(6)-C(5)-N(1)-C(3)$ & $169.3(3)$ \\
\hline $\mathrm{O}(2)-\mathrm{C}(2)-\mathrm{C}(3)-\mathrm{C}(4)$ & $-76.2(4)$ & $C(2)-C(3)-N(1)-C(5)$ & $-70.6(4)$ \\
\hline $\mathrm{O}(3)-\mathrm{C}(5)-\mathrm{C}(6)-\mathrm{N}(2)$ & $-37.4(5)$ & $C(4)-C(3)-N(1)-C(5)$ & 166.2(3) \\
\hline$N(1)-C(5)-C(6)-N(2)$ & $146.8(3)$ & $O(5)-C(11)-N(2)-C(10)$ & $2.6(6)$ \\
\hline $\mathrm{O}(3)-\mathrm{C}(5)-\mathrm{C}(6)-\mathrm{C}(7)$ & $75.2(4)$ & $O(4)-C(11)-N(2)-C(10)$ & $-178.9(3)$ \\
\hline$N(1)-C(5)-C(6)-C(7)$ & $-100.6(4)$ & $O(5)-C(11)-N(2)-C(6)$ & $169.2(4)$ \\
\hline$N(2)-C(6)-C(7)-C(9)$ & $11.3(4)$ & $\mathrm{O}(4)-\mathrm{C}(11)-\mathrm{N}(2)-\mathrm{C}(6)$ & $-12.3(5)$ \\
\hline$C(5)-C(6)-C(7)-C(9)$ & $-106.7(4)$ & $C(9)-C(10)-N(2)-C(11)$ & $-167.3(3)$ \\
\hline$N(2)-C(6)-C(7)-C(8)$ & $-51.8(4)$ & $C(9)-C(10)-N(2)-C(6)$ & $24.6(4)$ \\
\hline$C(5)-C(6)-C(7)-C(8)$ & $-169.8(4)$ & $C(7)-C(6)-N(2)-C(11)$ & $169.4(4)$ \\
\hline$C(6)-C(7)-C(8)-C(9)$ & $94.5(4)$ & $C(5)-C(6)-N(2)-C(11)$ & $-74.1(4)$ \\
\hline$C(7)-C(8)-C(9)-F(1)$ & $112.2(4)$ & $C(7)-C(6)-N(2)-C(10)$ & $-23.0(4)$ \\
\hline$C(7)-C(8)-C(9)-C(10)$ & $-97.6(4)$ & $C(5)-C(6)-N(2)-C(10)$ & 93.5(4) \\
\hline$C(6)-C(7)-C(9)-F(1)$ & $140.2(4)$ & $O(1)-C(2)-O(2)-C(1)$ & $0.9(7)$ \\
\hline$C(8)-C(7)-C(9)-F(1)$ & $-107.9(5)$ & $C(3)-C(2)-O(2)-C(1)$ & $-179.6(4)$ \\
\hline$C(6)-C(7)-C(9)-C(8)$ & $-111.9(4)$ & $O(5)-C(11)-O(4)-C(12)$ & $4.1(6)$ \\
\hline$C(6)-C(7)-C(9)-C(10)$ & $3.1(4)$ & $N(2)-C(11)-O(4)-C(12)$ & $-174.3(3)$ \\
\hline$C(8)-C(7)-C(9)-C(10)$ & $115.0(4)$ & $C(14)-C(12)-O(4)-C(11)$ & $-66.1(5)$ \\
\hline$F(1)-C(9)-C(10)-N(2)$ & $-156.4(3)$ & $C(13)-C(12)-0(4)-C(11)$ & $175.4(3)$ \\
\hline$C(8)-C(9)-C(10)-N(2)$ & $52.2(5)$ & $C(15)-C(12)-O(4)-C(11)$ & $57.7(5)$ \\
\hline
\end{tabular}


Table S15. Calculated reflections from PowderCell*

\begin{tabular}{|c|c|c|c|c|c|c|}
\hline $\mathrm{h}$ & $k$ & I & $2 \theta /^{\circ}$ & $\mathrm{d} / \AA$ & I/rel. & $|F(h k l)|$ \\
\hline 0 & 1 & 1 & 7.54 & 11.71 & 3.69 & 12.89 \\
\hline 0 & 0 & 2 & 10.04 & 8.81 & 76.35 & 110.65 \\
\hline 0 & 2 & 0 & 11.27 & 7.84 & 100.00 & 142.40 \\
\hline 0 & 2 & 1 & 12.35 & 7.16 & 4.39 & 23.14 \\
\hline 1 & 0 & 1 & 14.49 & 6.11 & 21.48 & 60.18 \\
\hline 1 & 1 & 0 & 14.71 & 6.02 & 5.95 & 32.18 \\
\hline 0 & 2 & 2 & 15.12 & 5.86 & 5.77 & 32.56 \\
\hline 1 & 1 & 1 & 15.55 & 5.69 & 31.81 & 55.68 \\
\hline 1 & 0 & 2 & 16.92 & 5.24 & 50.45 & 108.07 \\
\hline 0 & 3 & 1 & 17.68 & 5.01 & 4.41 & 33.45 \\
\hline 1 & 2 & 0 & 17.69 & 5.01 & 26.98 & 82.71 \\
\hline 1 & 1 & 2 & 17.84 & 4.97 & 47.11 & 77.99 \\
\hline 1 & 2 & 1 & 18.39 & 4.82 & 64.49 & 94.15 \\
\hline 0 & 2 & 3 & 18.87 & 4.70 & 40.08 & 107.75 \\
\hline 0 & 3 & 2 & 19.73 & 4.50 & 5.06 & 40.11 \\
\hline 0 & 0 & 4 & 20.15 & 4.40 & 3.40 & 47.48 \\
\hline 1 & 0 & 3 & 20.35 & 4.36 & 15.63 & 72.75 \\
\hline 1 & 2 & 2 & 20.38 & 4.35 & 33.83 & 75.78 \\
\hline 0 & 1 & 4 & 20.94 & 4.24 & 17.62 & 79.57 \\
\hline 1 & 1 & 3 & 21.13 & 4.20 & 4.99 & 30.21 \\
\hline 1 & 3 & 0 & 21.78 & 4.08 & 2.47 & 31.02 \\
\hline 1 & 3 & 1 & 22.36 & 3.97 & 8.69 & 42.32 \\
\hline 0 & 3 & 3 & 22.76 & 3.90 & 18.58 & 89.10 \\
\hline 0 & 4 & 1 & 23.22 & 3.83 & 11.63 & 71.99 \\
\hline 1 & 2 & 3 & 23.32 & 3.81 & 9.33 & 45.80 \\
\hline 1 & 3 & 2 & 24.03 & 3.70 & 8.36 & 44.75 \\
\hline 0 & 4 & 2 & 24.84 & 3.58 & 2.48 & 35.67 \\
\hline 1 & 1 & 4 & 25.04 & 3.55 & 6.29 & 40.51 \\
\hline 0 & 3 & 4 & 26.45 & 3.37 & 5.50 & 56.75 \\
\hline 1 & 3 & 3 & 26.60 & 3.35 & 5.95 & 42.00 \\
\hline 1 & 2 & 4 & 26.94 & 3.31 & 9.51 & 53.81 \\
\hline 1 & 4 & 1 & 27.00 & 3.30 & 2.49 & 27.59 \\
\hline
\end{tabular}




\begin{tabular}{|lll|l|l|l|l|}
\hline 2 & 0 & 1 & 27.84 & 3.20 & 8.66 & 75.18 \\
\hline 2 & 1 & 0 & 27.96 & 3.19 & 6.92 & 67.53 \\
\hline 1 & 4 & 2 & 28.41 & 3.14 & 14.71 & 70.83 \\
\hline 2 & 1 & 1 & 28.42 & 3.14 & 2.61 & 29.82 \\
\hline 1 & 0 & 5 & 28.79 & 3.10 & 3.19 & 47.28 \\
\hline 1 & 1 & 5 & 29.36 & 3.04 & 4.54 & 40.75 \\
\hline 2 & 1 & 2 & 29.77 & 3.00 & 6.75 & 50.42 \\
\hline 1 & 3 & 4 & 29.84 & 2.99 & 3.56 & 36.73 \\
\hline
\end{tabular}

Source: $\mathrm{Cu}-\mathrm{K}_{\alpha 1}(\lambda=1.540598 \AA \AA)$

Condition on reflections: $1 \geq 2$

Range (20): From $3^{\circ}$ to $30^{\circ}$

*PowderCell for Windows (version 2.4) by Kraus W. \& Nolze G., Federal institute for materials Research and testing, Rudower Chausse 5, 12489 Berlin Germany. 